\title{
Atomic and molecular defects in solid ${ }^{4} \mathrm{He}$
}

\author{
P. Moroshkin A. Hofer A. Weis \\ Physics Department, Université de Fribourg, Chemin du Musée 3, 1700 Fribourg, Switzerland
}

\section{Introduction}

Matrix isolation spectroscopy has been used since 1950s to study highly reactive atomic and molecular species. Rare gas solids are typically used in those studies because of their chemical inertness, and the low temperature required for the matrix formation is an additional asset for the studies. In the present paper we review experiments that have extended the matrix isolation technique to the use of solid ${ }^{4} \mathrm{He}$ as the matrix material. Those studies were initiated by one of the authors (AW) together with Sergei Kanorsky in 1994 at the Max Planck Institute for Quantum Optics in Garching (Germany). They were later continued at the Institute for Applied Physics at the University of Bonn and are pursued, since 2000, at the Physics Department of the University of Fribourg (Switzerland). The studies of impurities in solid helium were reviewed in [1,2] and more recently in [3]. This field of solid helium matrix isolation spectroscopy is closely connected with the studies of atomic and molecular impurities in bulk liquid He (reviewed, e.g., in [4]) and in He nano-droplets. The latter research domain was pioneered by the teams of J. P. Toennies in Göttingen and G. Scoles at Princeton in 1993 and has been rapidly expanding since with review articles of the topic being published on average every 2 to 3 years: [5-8]. There is also a very strong connection with studies of defects formed by injected free electrons in liquid and solid He, a research initiated in the 1960s which has remained an active area of research up to date. In fact, the concept of atomic bubbles which forms the basis for the theoretical understanding of atomic defect structures in condensed helium, was first developed in those early studies of electrons in condensed helium. The present paper updates and extends our previous review [3] and presents several new important studies, with the goal of giving a comprehensive picture of today's knowledge - both experimental and theoretical - on atomic, molecular and electronic defect structures in solid and liquid helium. We will also give a short review of related studies of metal atoms isolated in heavier rare-gas matrices thereby updating an existing review article from 1999 [9]. Several common aspects of matrix isolation in He and in heavier rare gases will be outlined and the specific peculiarities of solid He will be stressed. We also include a chapter on impurity-helium solids bound by van der Waals forces discovered in 1974 by E. B. Gordon and coworkers whose properties were reviewed in [10-12]. With a few exceptions $[2,13]$ the latter research domain is usually considered isolated from other studies 

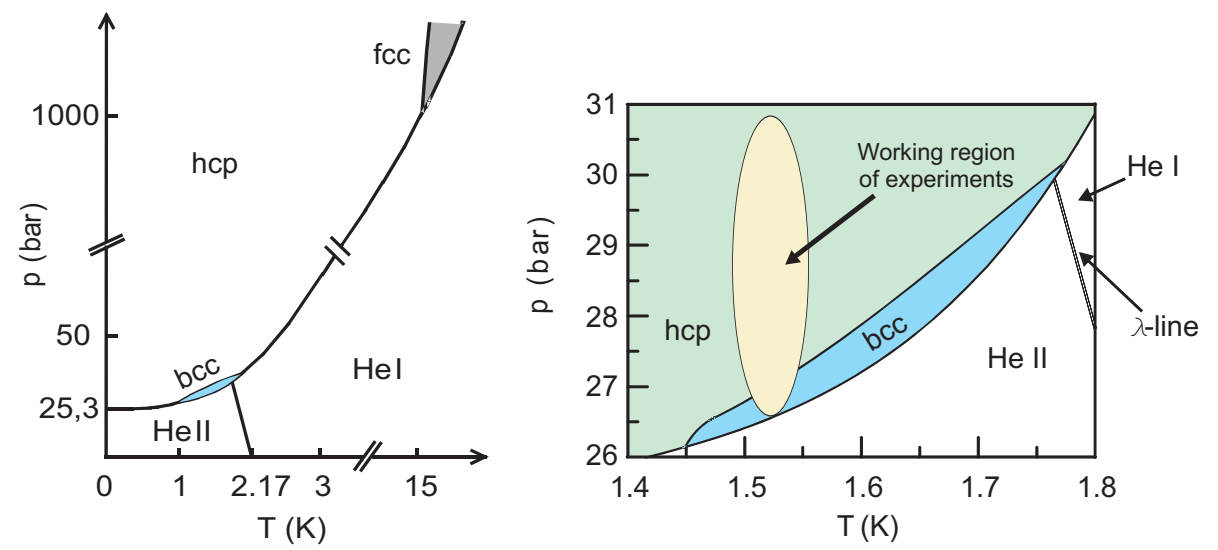

Fig. 1. ${ }^{4} \mathrm{He}$ phase diagram. HeI and HeII denote the normal fluid and the superfluid phase, respectively. The solid phases are labeled according to their crystalline structures: bcc for body-centered cubic, hcp for hexagonally close-packed, and fcc for face-centered cubic. The $p-T$ region studied in our experiments is shown on the right panel.

of impurities in condensed helium. However, our recent discovery of solid He stabilized by impurity ions points towards a much closer relationship between the two approaches.

\section{Solid ${ }^{4} \mathrm{He}$}

Helium $\left({ }^{4} \mathrm{He}\right)$ is a unique substance that becomes superfluid at low temperatures and that solidifies only under an external pressure in excess of 25 bar, even at the absolute zero of temperature (see Fig. 1). Both features are manifestations of the macroscopic quantum nature of condensed helium which is due to the smallness of the He mass and the weakness of the interatomic He-He interaction. The de Broglie wavelength of a He atom $\lambda_{B}=h / \sqrt{2 \pi m_{H e} k T}$ is very large and at low temperatures it becomes comparable to the interatomic separation in condensed helium $(R \approx 3.7 \AA$ at the solidification pressure at $1.5 \mathrm{~K})$. The He atoms are strongly delocalized (even in the crystalline phase) and form a so-called quantum liquid or quantum solid because of the large overlap of the wavefunctions which makes condensed, i.e., liquid or solid ${ }^{4} \mathrm{He}$ a macroscopic quantum object. The degree of delocalization is best characterized by the Lindemann ratio $\gamma=\sqrt{\left\langle u^{2}\right\rangle} / R$ or by the de Boer parameter $\Lambda=\lambda_{B} /(2 \pi \sigma)$, in which $\sqrt{\left\langle u^{2}\right\rangle}$ is the rms value of the vibrational amplitude of an atom in the matrix and $\sigma$ is the distance at which the interatomic potential passes through zero. For solid He, close to the solidification point both parameters are approximately equal to 0.3 , much larger than for any other quantum solid [14].

The interatomic interaction in helium is very close to that of hard spheres (Fig. 2). The potential well at $2.9 \AA$ has a depth of only $5.4 \mathrm{~cm}^{-1}[15]$. It is due to the induced dipole-dipole interaction, which is extremely weak in helium because of its small electric polarizability. At smaller interatomic separations the interaction is strongly repulsive since it is dominated by the Pauli repulsion between the closed electronic $S$-shells. The localization of He atoms in a crystalline lattice requires a zero-point energy $E_{Z}$ significantly larger than the well depth in the pair potential. The ratio of the kinetic and potential energies of an atom in the crystal is another measure of the quantum properties of the solid. For He this ratio is larger than 1 , while for all other rare gas solids is smaller than 1 . 


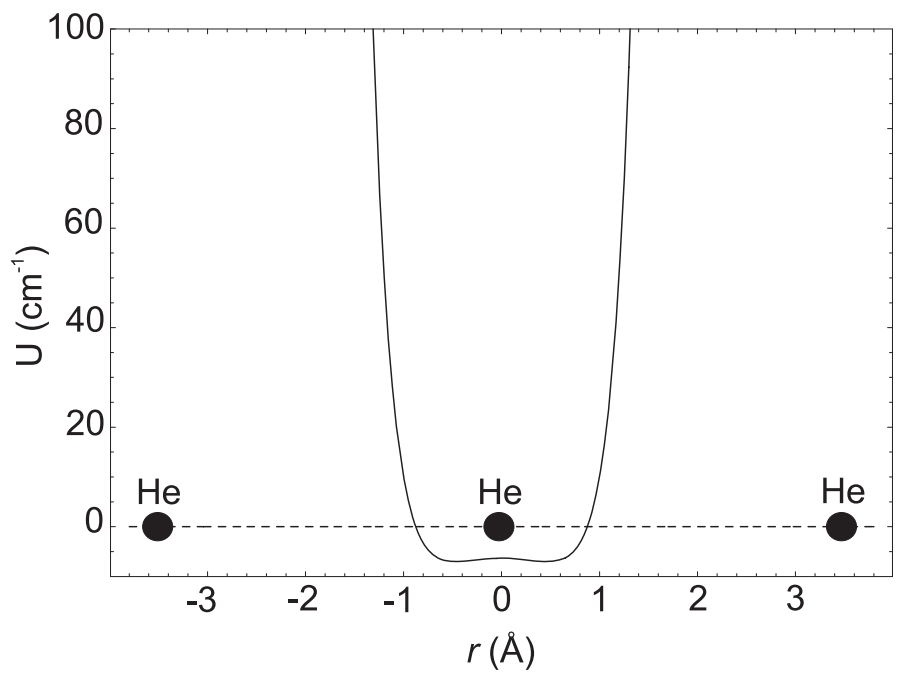

Fig. 2. The potential energy of a He atom interacting with two neighboring atoms in the crystal (internuclear separation 3.5 $\AA$ ) as a function of its displacement from the equilibrium position. The interatomic pair potential is taken from [15].

The properties of solid He were studied in great detail [16]. In the range of temperatures and pressures relevant for the present review $(T=1-2 \mathrm{~K}, p=25-100$ bar) there exist two crystalline phases of ${ }^{4} \mathrm{He}$ (see Fig. 1): hexagonal close-packed (hcp) and the body-centered cubic (bcc) phase. The crystalline structures and the corresponding interatomic spacings were obtained by $X$-ray and neutron scattering data. Liquid He and both solid phases are highly compressible because of their quantum properties. The elastic constants of bcc and hcp solid He were obtained from measurements of the orientation-dependent sound velocity $[17,18]$. Recently evidence for a supersolid phase of ${ }^{4} \mathrm{He}$ at temperatures below $0.2 \mathrm{~K}$ was obtained [19] and is under active discussion [20].

The molar volume $\nu$ of condensed He as a function of pressure and temperature is shown in Fig. 3 [21]. For liquid He we use the experimental data from [22]. For the hcp solid $\nu(p, T)$ is obtained by fitting the experimental data $(\partial p / \partial T)_{\nu}$ from [23] by a polynomial of a type $\sum_{m, n} a_{m n} \nu^{m} T^{n}$. For the small island representing the bcc phase the molar volume is obtained by assuming a constant compressibility (taken from [24]) and by using the measured values of $\nu$ along the melting line from [25].

The density of solid He at the melting point at $1.5 \mathrm{~K}$ is approximately $7 \%$ higher than that of liquid He under the same conditions. Condensed He is transparent in all spectral ranges explored in the experiments reported here (UV, visible, IR, and microwave). The boundary between the liquid and the solid phase as well as between the two solid phases can be seen by eye due to their different indices of refraction (as shown in Fig. 35).

\section{The atomic bubble model}

\subsection{Electron bubbles}

The structure of the trapping sites of many atomic and molecular impurities in liquid or solid He is well described by the so-called bubble model. This approach was introduced by Jortner et al. [26] to model individual electrons in liquid He for which the expression "electron 


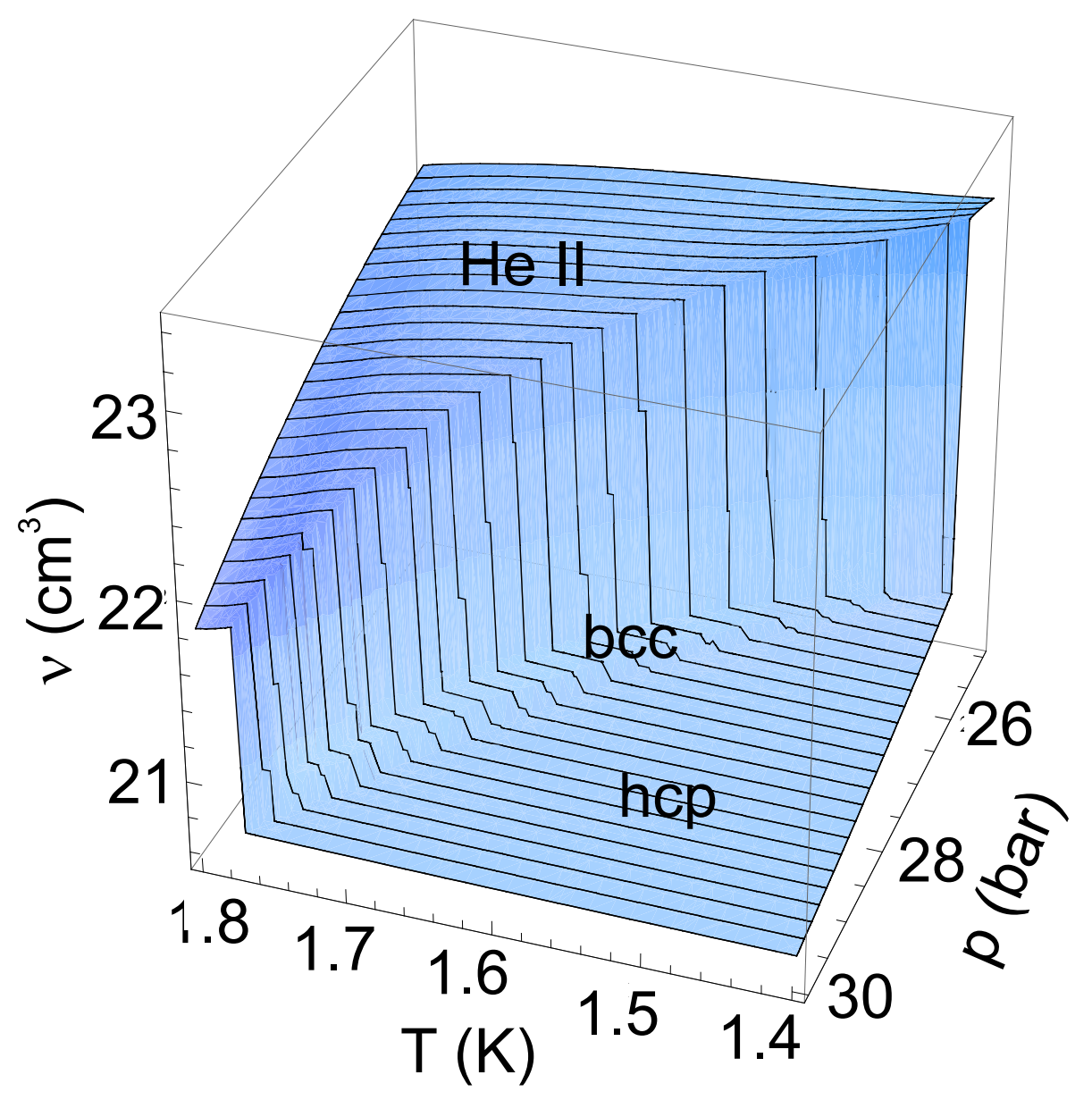

Fig. 3. Molar volume of condensed ${ }^{4} \mathrm{He}$ as function of helium pressure and temperature [21].

bubble" was coined. In this model the quantum many-body problem of the impurity-helium interaction is greatly simplified by using two fundamental properties of condensed helium. First, due to its quantum nature, i.e., the strong overlap of the helium-atom wave functions, condensed helium can be treated as a continuous medium even at the nanometer scale. Second, the interaction between an excess electron and the closed $S$-shells of surrounding He atoms is strongly repulsive due to the Pauli principle and its strength greatly exceeds the interaction strength between neighboring He atoms in the lattice. The repulsion of helium by the dopant exerts an internal pressure by which helium atoms are expelled from the volume occupied by the excess electron and form a small spherical cavity, called electron bubble.

The size of the bubble is determined by the balance between the electron-helium repulsion and the external pressure on the bubble surface. The equilibrium configuration can be found by minimizing the total energy of the impurity plus bubble system given by

$$
E_{t o t}=E_{\text {int }}+p V_{\text {bubble }}+\sigma S_{b u b b l e}+\frac{\hbar}{8 M} \int \frac{(\nabla \rho(\mathbf{R}))^{2}}{\rho(\mathbf{R})} d^{3} \mathbf{R}
$$

The first term in Eq. 1 represents the interaction between the dopant and the matrix. The energy of the bubble in condensed helium is composed of the pressure-volume work, $p V_{b u b b l e}$, the energy due to surface tension, $\sigma S_{b u b b l e}$, and the volume kinetic energy, i.e., the excess 
zero-point energy due to the localization of the He atoms at the bubble interface, given by the fourth term of Eq. 1, with the He density distribution $\rho(\mathbf{R})$.

The electron energy $E_{\text {int }}$ is found by solving the Schrödinger equation

$$
\left[-\frac{\hbar^{2}}{2 m} \nabla^{2}+V(\mathbf{r})\right] \phi(\mathbf{r})=E \phi(\mathbf{r})
$$

The electron-helium interaction is described by a pseudo-potential $V_{p s}(\mathbf{r}, \mathbf{R})$ which has to be integrated over the bubble

$$
V(r)=\int d^{3} R \rho(\mathbf{R}) V_{p s}(\mathbf{r}, \mathbf{R})
$$

For a spherically symmetric defect, the helium density $\rho(\mathbf{R})$ is described by the radial trial function

$$
\rho\left(R, R_{0}, \alpha\right)=\left\{\begin{array}{ll}
0 & R<R_{0} \\
\rho_{0}\left[1-\left\{1+\alpha\left(R-R_{0}\right)\right\} e^{-\alpha\left(R-R_{0}\right)}\right] & R \geq R_{0}
\end{array},\right.
$$

where $\rho_{0}$ is the bulk helium density and the parameters $R_{b}$ and $\alpha$ are measures of the bubble radius and the bubble interface thickness, respectively, which are adjusted to minimize the total defect energy $E_{t o t}$.

The further development of the electron bubble model [27-29] included a refined description of the surface tension and the volume kinetic energy, as well as the polarization of He atoms by the embedded electron that gives an additional contribution $V_{p o l}(\mathbf{r}, \mathbf{R})$ to the energy of the defect. More recently a hydrodynamic model $[30,31]$ was presented that treats the dynamics of the electron bubble formation in liquid He. The results of numeric calculations $[32,33]$ using the density functional technique are in good agreement with those of the bubble model.

The characteristic radius $R_{b}$ of the electron bubble, usually defined as the center of gravity of the bubble interface function $\rho\left(R, R_{0}, \alpha\right)$ in liquid He at saturated vapor pressure is about $17 \AA$. The bubbles are highly compressible and shrink to $11 \AA$ when increasing the pressure to 25 bar. The size of the bubble explains the relatively low mobilities of the electrons observed in liquid helium [34,35]. The bubble model also allows one to calculate energy levels of the electron (bound in the potential well) [36-38]. The transitions between the $1 s$ ground state and the excited $1 p$ and $2 p$ states lie in infrared part of the spectrum. These transitions have been observed experimentally and agree with the predictions of the bubble model (see Sec. 7.1).

\subsection{Atomic bubbles}

The valence electron in an atom has a stronger localization than a free electron due to its attractive interaction with the atomic core and the resulting atomic bubble is smaller than the (pure) electron bubble discussed above. For the same reason the atomic bubble is also less compressible than the electron bubble. In order to account for the interaction of the valence electron with the atomic core, a term $V_{C s}$ has to be added to the right hand side of Eq. 3. A further contribution $V_{c c}$ comes from the polarization of He atoms by the atomic 
core of the alkali atom, so that the potential seen by a valence electron moving under the influence of the alkali core and the surrounding He atoms reads

$$
V(\mathbf{r})=V_{C s}+\int d^{3} R \rho(\mathbf{r}, \mathbf{R})(\mathbf{R})\left[V_{c c}(\mathbf{R})+V_{p o l}(\mathbf{r}, \mathbf{R})+V_{p s}(\mathbf{r}, \mathbf{R})\right]
$$

First calculations [39-41] of atomic bubbles were performed for excited ${ }^{4} \mathrm{He}$ atoms in liquid He. For the metastable $2^{3} S$ state a spherical bubble with a radius of $5.3 \AA$ was obtained by assuming that the electronic wavefunction of the atom in the bubble is unperturbed. This metastable state is the lower level in optical absorption and emission experiments. For the higher excited states $3{ }^{1} S$ and $3^{3} S$ the perturbation of the wavefunction is more significant and was taken into account when minimizing the energy of the atom plus bubble system. The wavelengths and spectral lineshapes of the $2^{3} S-2^{3} P$ transition in absorption and of the $3^{3} S-2^{3} P$ and $3^{1} S-2^{1} P$ transitions in emission were calculated and were demonstrated to yield a good agreement with experiments (see Sec. 7.2).

The first calculations $[42,43]$ of bubbles formed by impurity atoms were performed for the alkaline-earth elements $\mathrm{Ca}, \mathrm{Sr}$, and $\mathrm{Ba}$ for which the interaction energy $E_{\text {int }}$ was computed using the electronic wavefunctions of the free atom. The energies of electronic transitions derived from those calculations yielded much larger blueshifts than the experimentally observed shifts.

The valence electron in the ground state of an alkali atom is loosely bound in a spherically symmetric $n S_{1 / 2}$ orbital and its interaction with condensed helium is very similar to that of a free electron. Extensive calculations of the atomic bubbles formed by alkali (Rb and $\mathrm{Cs}$ ) atoms in liquid He were reported by Kinoshita et al. [44-46]. The authors used an expansion of the perturbed electronic wavefunctions of the dopant's valence electron in the basis of unperturbed $\left|L, M_{L}, S, M_{S}\right\rangle$ states including only the lowest $n S$ and $n P$ states. At saturated vapor pressure they obtained equilibrium bubble radii $R_{b}(6 S)=6.5 \AA$ for the Cs ground state and $R_{b}(6 P)=7.9 \AA$ for its first excited state. The calculated $n S_{1 / 2} \rightarrow n P_{1 / 2}$ transition energies for $\mathrm{Rb}$ and $\mathrm{Cs}$ agree well with the experimental results.

The Schrödinger equation (Eq. 2) for the valence electron has been solved numerically in [48] for the $6 S_{1 / 2}$ and $6 P_{1 / 2}$ states of $\mathrm{Cs}$ in spherical bubbles for which the angular and radial variables can be separated due to the spherical symmetry of the problem. The authors of [48] obtained a self-consistent solution for the eigenenergies and electronic wavefunctions of the Cs atom in the bubble. In Fig. 4 we compare these solutions with the radial wavefunctions of the free Cs atom. The bubble parameters correspond to the ground (Fig. 4 (a) and (b)) and excited (Fig. 4 (c)) states. In the same figure the He density distribution $\rho(R)$ is shown for both cases. These wavefunctions were used to model various experimental observables, such as the hyperfine splitting of the ground state, the radiative lifetime of the excited $6 P_{1 / 2}$ state, the absorption and emission spectra of the $6 S_{1 / 2}-6 P_{1 / 2}$ transition. In all cases a good agreement with experimental results was found (see Sec. 7.3, 8.1 and 9.5) thus consolidating the validity of the bubble model.

In many cases the interaction between the impurity atom and the He atoms can be described by ab initio pair potentials $U(\mathbf{R})$ given in the literature. In particular the alkalihelium pair potentials have been calculated by Pascale $[49,50]$. The calculations of atomic bubbles can be significantly simplified by writing the impurity-helium interaction energy as 

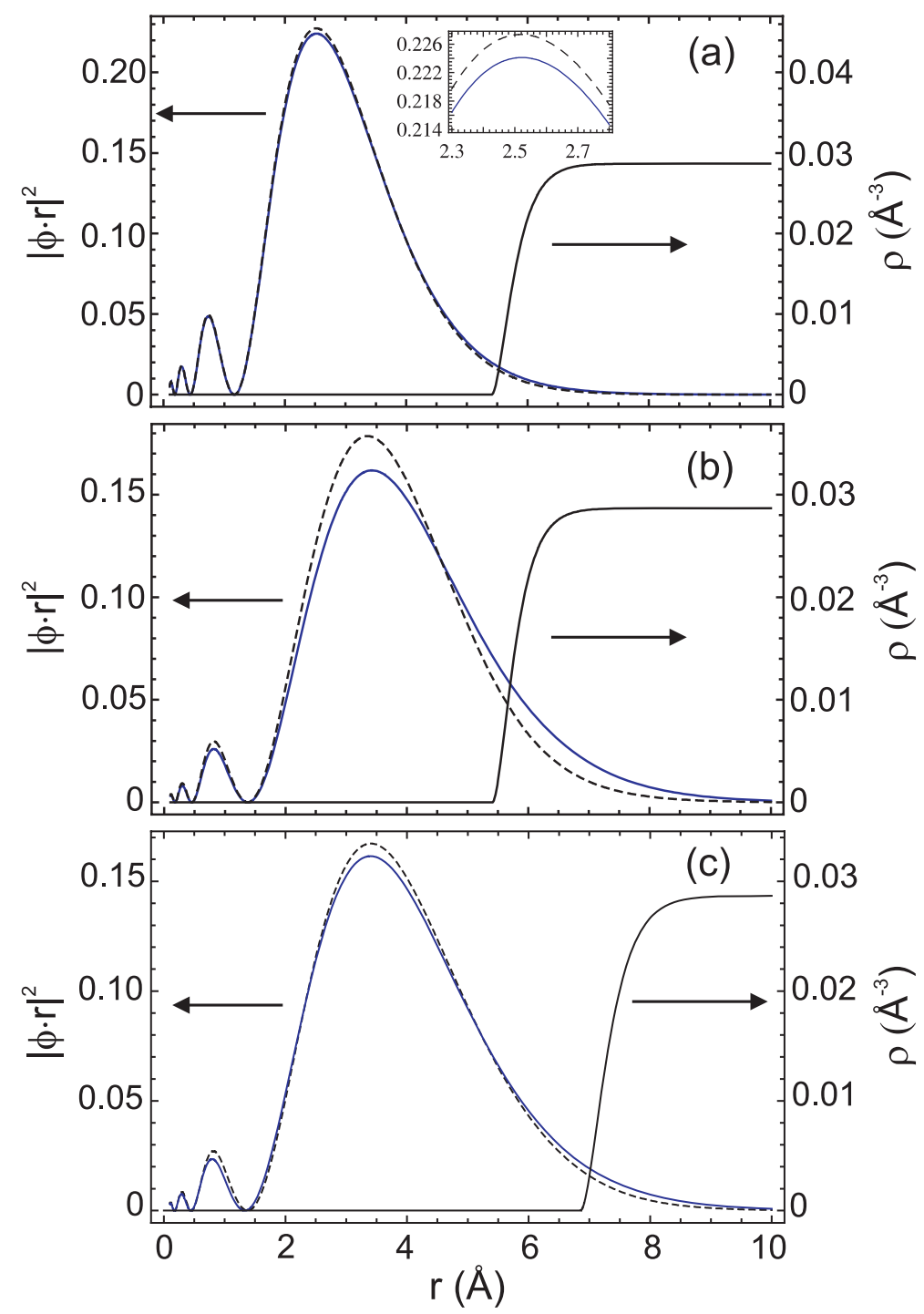

Fig. 4. Valence electron density of a free Cs atom (solid blue lines) and a Cs atom in a bubble (dashed lines). The corresponding He density distributions $\rho(R)$ are shown as solid black lines. (a) Cs in the $6 S_{1 / 2}$ state and (b) Cs in the $6 P_{1 / 2}$ state located in a bubble corresponding to the equilibrium with the $6 S_{1 / 2}$ ground state; (c) $6 P_{1 / 2}$ state in a bubble corresponding to the equilibrium with the $6 P_{1 / 2}$ excited state.

$$
E_{\text {int }}=\int U(\mathbf{R}) \rho(\mathbf{R}) d^{3} \mathbf{R} .
$$

The pair potential $U(\mathbf{R})$ depends on the electronic state of the dopant which is, in general, non-isotropic for states with a non vanishing orbital momentum $L$. As a consequence the equilibrium bubble shape may be non-spherical. Moreover, as discussed below in Sec. 4.1, non spherical bubbles may exist even around spherically-symmetric electronic states due to the anisotropy of the elastic properties of the matrix (in solid helium), or due to the oscillations of the bubble interface (in liquid helium). In that situation the valence electron of the dopant moves in a nonspherical potential and the variables in the Schrödinger equation (Eq. 2) can no longer be separated. Using pair potentials thus greatly simplifies the calculation in those cases. For a given $L$ the interatomic interaction is characterized by a set of potentials 


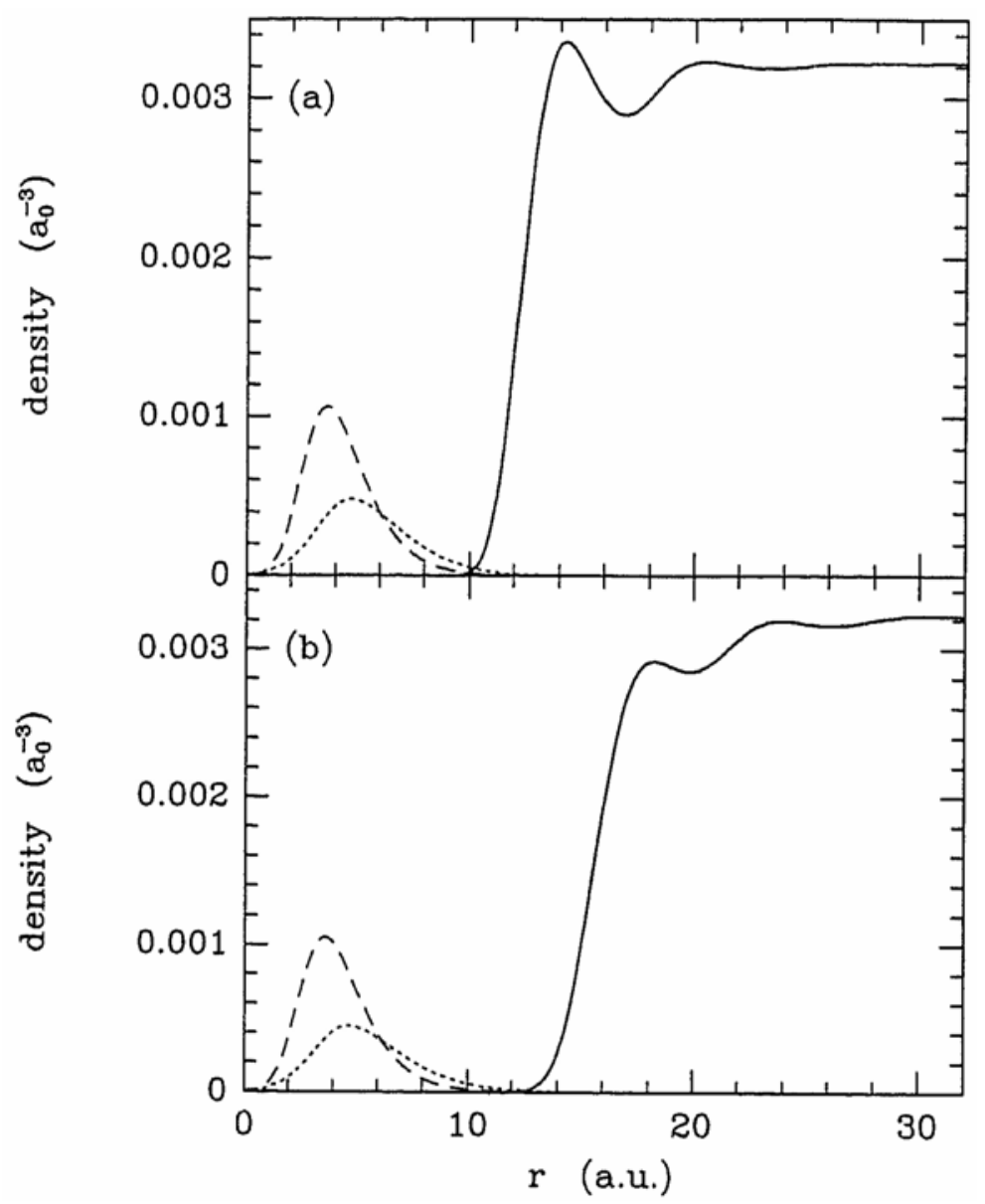

Fig. 5. He density distributions $\rho(R)$ around a Cs atom in the $6 S_{1 / 2}$ ground state (a) and in the $6 P_{1 / 2}$ excited state (b) obtained by density functional calculations [47] (solid lines). Dashed and dotted lines show the valence electron density for the $6 S_{1 / 2}$ and $6 P_{1 / 2}$ state respectively. Reproduced from [47] with kind permission of Springer Science and Business Media.

$U_{L, M_{L}}(R)$. For each atomic pair the quantization axis is taken along the internuclear axis and the interaction Hamiltonian $H_{\text {diatom }}(R)$ is represented by a diagonal $2 L+1 \times 2 L+1$ matrix with diagonal elements equal to $U_{L, M_{L}}(R)$ ( $+M_{L}$ and $-M_{L}$ states being degenerate). The system of coordinates is then rotated in such a way, that the new $z$ axis coincides with the quantization axis chosen for the bubble. Under this rotation the Hamiltonian matrix is transformed according to

$$
\tilde{H}_{\text {diatom }}(R, \theta, \varphi)=D_{L}^{*}(\theta, \varphi) H_{\text {diatom }}(R) D_{L}(\theta, \varphi),
$$

where $D_{L}(\theta, \varphi)$ are Wigner rotation matrices. The total interaction Hamiltonian $H_{\text {int }}$ is obtained by an integration of the pair interactions, weighted with a He density distribution that reflects the bubble configuration, as in Eq. 6. Finally, the eigenenergies of the atom in the bubble are obtained by diagonalizing $H_{\text {int }}$ and adding the bubble energy (Eq. 1). This approach was used in a number of publications, including earlier works on $\mathrm{Cs}$ in liquid and solid helium [51,1], as well as for the alkaline-earth elements $\mathrm{Mg}$ [52-54], Ca [52,55,56], $\mathrm{Sr}$ [52], and $\mathrm{Ba}[57,51,1,52,55]$, and $\mathrm{Yb}^{+}$ions [58]. 
The trapping site structures of $\mathrm{Na}$, $\mathrm{Cs}$, and $\mathrm{Al}$ in liquid He were also modeled using Monte Carlo and density functional techniques treating helium atoms as individual objects $[47,59,60]$. The results of these simulations for the spherically symmetric ground states of alkalis are very close to that of a bubble model calculations. The helium density profiles at the bubble interface obtained in [47] are shown in Fig. 5. One can see that the more sophisticated density functional calculations show a helium shell structure around the impurity that is not taken into account by the simple function Eq. 4 usually used to model the bubble interface (see Fig. 4).

\subsection{Electronic transitions in atomic bubbles}

Accordingly to the Frank-Condon principle, the shape and the size of the bubble do not change during the electronic transition since the transition occurs on a time scale shorter than the bubble oscillation period. Once the embedded atom is excited the bubble relaxes to a larger radius that reflects the larger extension of the excited state wavefunction. Assuming that the bubble expansion proceeds at the speed of sound, one can estimate that this relaxation occurs on a picosecond time scale. The fluorescence transition occurs in the larger bubble in which the excited state lives for a few ten ns, close to the free atomic lifetime.

In order to calculate the lineshapes of the optical absorption lines of atomic bubbles, the following procedure has been applied $[42-45,55,48]$. The broadening of the atomic spectral lines is attributed to the spatial extension of the electronic wavefunctions due to radial (breathing mode) bubble oscillations. The probability distribution for finding a bubble with radius $R$ is given by $|\Phi(R)|^{2}$, where $\Phi(R)$ is the wavefunction associated with the bubble vibration. It is found by solving the one-dimensional Schrödinger equation

$$
\left[-\frac{\hbar^{2}}{2 M_{b}} \frac{d^{2}}{d R^{2}}+E_{t o t}(R)\right] \Phi(R)=E_{b} \Phi(R)
$$

where $E_{\text {tot }}(R)$ is the total energy of the atom plus bubble system (Eq. 1) that depends on the oscillating bubble radius $R . M_{b}=4 \pi R_{b}^{3} \rho_{0} m_{H e}$ is the hydrodynamic mass of the bubble and $E_{b}$ the vibration eigenenergy. The calculations $[45,48]$ have shown that the splitting between the vibrational ground state and the first excited vibrational level is equivalent to several Kelvin, so that at the temperature $T \approx 1.5 \mathrm{~K}$ around which most experiments are preformed only the lowest vibrational state is populated. To each bubble radius $R$ corresponds a given transition energy with a relative weight given by $|\Phi(R)|^{2}$. An equivalent procedure can be applied for calculating the emission spectra. In that case one starts from breathing mode oscillations of the bubble corresponding to the excited state.

A similar technique was applied $[61,62]$ to model the spectral profile of the $1 s \rightarrow 1 p$ absorption line of electron bubbles in liquid He, taking into account not only breathing but also quadrupolar bubble oscillation modes.

Another approach for the calculation of the absorption and emission lineshapes may be used in the case, when the impurity-helium pair potentials are known $[57,1,51,54]$. Instead of considering the collective motion of He atoms (bubble oscillations), one can treat the He atoms as independent perturbers and apply the standard adiabatic line-broadening theory [41]. In this approach the lineshapes are calculated from the Fourier transform of the optical dipole autocorrelation function $C(\tau)$ represented by 


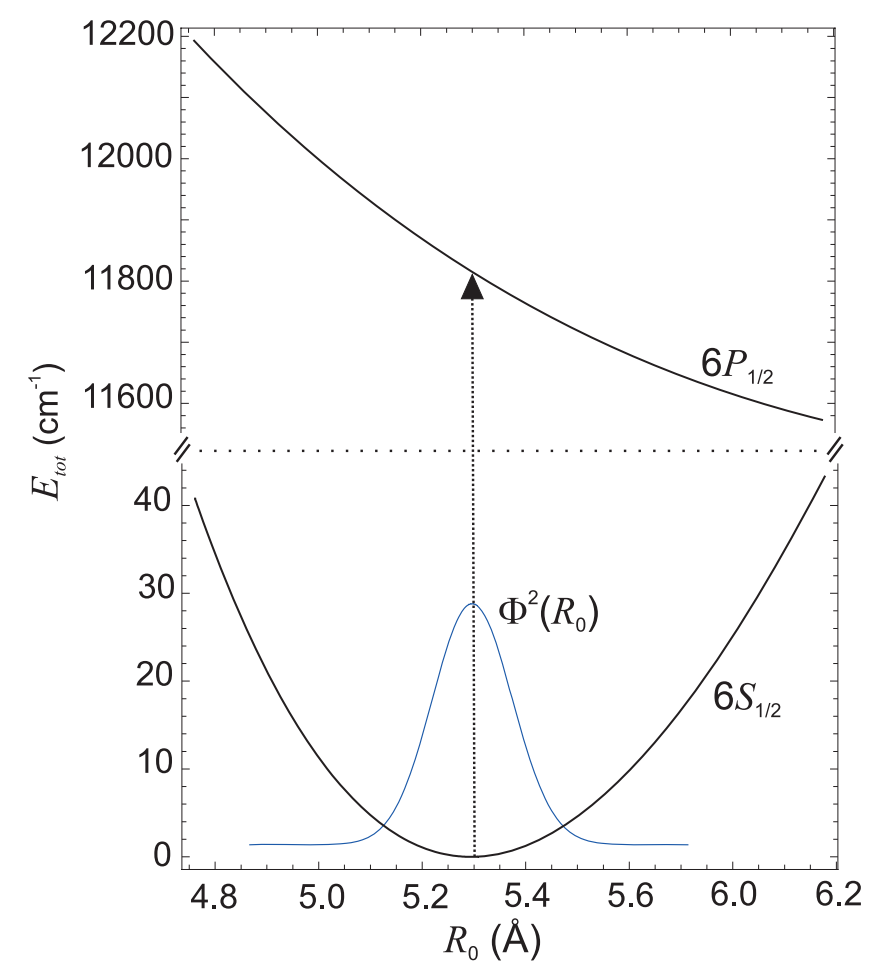

Fig. 6. Calculated potential energy (solid black line) of the Cs $6 S_{1 / 2}$ and $6 P_{1 / 2}$ states in a spherical bubble including the bubble energy as a function of the bubble radius $R_{0}$ together with the probability distribution $\Phi\left(R_{0}\right)^{2}$ shown as a blue curve.

$$
C(\tau)=\exp \left\{-\int(1-\exp [-i \Delta \nu(R) \tau]) \rho(R) d^{3} R\right\}
$$

where $\Delta \nu(R)$ is the shift of the dopant's transition energy due to a single He atom located at position $R$ and $\rho(R)$ gives the probability to find a He atom at this position.

A combination of the two methods outlined above was used in [58,56], where even higher order (quadrupolar) oscillation modes were taken into account.

\subsection{Atomic bubble model for isotropic solid He}

Electron bubbles in solid He were considered for the first time by Cohen and Jortner [63] who suggested that bubbles in solid helium should not differ significantly from bubbles in liquid He. Their estimation showed that the additional energy due to the strain produced in the He crystal - treated as an isotropic elastic solid - is negligible. This was confirmed by spectroscopic studies of electron bubbles in liquid $[64,65]$ and in solid He [66] which revealed no discontinuity of the optical transition frequencies at the phase transition. The first experiments with atomic barium in solid He $[67,57]$ showed that this conclusion also holds for atomic bubbles. However, detailed studies of the absorption and emission spectra of Cs atoms in liquid [44-46] and in solid [51,68,48] helium revealed a number of well pronounced effects that occur at the liquid-solid phase transition as well as at the transition from the body-centered cubic (bcc) to the hexagonal close-packed (hcp) crystalline phase. Analyzing the observed discontinuity of the $6 S_{1 / 2} \rightarrow 6 P_{1 / 2}$ transition wavelength at the liquid-bcc phase boundary, we have adapted the liquid atomic bubble model to bubbles in solid He 
[48] by a characteristic new term in the expression for the bubble energy.

As described in detail in Sec. 6.1, $[51,68,48]$ the doping of solid helium proceeds in two steps: injection of atoms into liquid helium (formation of liquid bubbles) followed by a solidification of the sample by heat exchange with the surrounding solid helium which induces the transition to the solid bubbles. In liquid He the equilibrium bubble radius $R_{b}$ is determined by the balance between the repulsive Cs-He interaction $E_{\text {int }}$ and the bubble energy which is minimized for $R_{0} \rightarrow 0$ (Eq. 1). The former contribution is proportional to the He density $\rho$ (see Eq. 5), while the latter is dominated by the $p V$ term and is thus proportional to the He pressure. At the liquid-solid phase boundary the helium density increases by $8 \%$ without any change in the helium pressure. The bubble thus tends to expand. In liquid He, the expansion of the bubble would simply displace surrounding helium at constant pressure. However, in the solid phase the bubble expansion proceeds via a compression of the solid around the bubble since the total sample volume is fixed. The resulting elastic force $F_{\text {elastic }}$ counteracts the force $F_{C s-H e}$ acting on the bubble interface from inside and a new equilibrium is established.

For the spherical bubble one obtaines [48]

$$
F_{\text {elastic }}=-F_{C s-H e}=-\left.\frac{\partial E_{\text {int }}}{\partial R_{0}}\right|_{R_{0}=R_{e q}},
$$

where $R_{e q}$ is the equilibrium bubble radius for a given electronic state of the atom. The deformation energy is then given by $F_{\text {elastic }} \Delta R$, where $\Delta R=R_{0}^{\text {solid }}-R_{0}^{\text {liquid }}$ is the difference between the equilibrium bubble radii in solid and in liquid He, and Eq. 1 defining the total defect energy becomes

$$
E_{\text {tot }}=E_{\text {int }}+\frac{4}{3} \pi R_{b}^{3} p+4 \pi R_{b}^{2} \sigma+\frac{\hbar}{8 M} \int \frac{(\nabla \rho(R))^{2}}{\rho(R)} d^{3} R-\left.\frac{\partial E_{\text {int }}}{\partial R_{0}}\right|_{R_{0}=R_{0}^{\text {liquid }}} \Delta R .
$$

The additional term leads to an equilibrium bubble size in bcc He which is slightly smaller than the bubble size in liquid and accounts for the marked jumps in the absorption and emission wavelengths at the liquid to solid (bcc) phase transition (see Sec. 7.3).

\section{Deformed bubbles}

\subsection{Anisotropy of elastic properties of solid He}

The treatment of atomic bubbles reported in [48] addressed only the spherically symmetric $6 S_{1 / 2}$ and $6 P_{1 / 2}$ states of Cs and assumed solid helium to be a continuous medium with isotropic elastic properties, so that the resulting bubbles obviously have a spherical symmetry. However, it is well known that the elastic properties of solids depend on their crystalline structure and are in general anisotropic. The generalized Hooke's law is usually written as

$$
\Sigma_{i j}=C_{i j k l} \varepsilon_{k l}
$$

where $\Sigma_{i j}$ and $\varepsilon_{k l}$ are the stress and strain tensors of rank 2, and where $C_{i j k l}$ is the stiffness tensor of rank 4. The explicit orientational dependence of Young's modulus $E(\mathbf{n})$ for cubic and hexagonal crystals can be expressed by compact analytical formulas [69] derived using the tensor formalism 


$$
\frac{1}{E(\mathbf{n})}=n_{i} n_{j} C_{i j k l}^{-1} n_{k} n_{l}
$$

where $\mathbf{n}$ is the unit vector specifying the direction. The angular dependence of $1 / E(\mathbf{n})$ for the crystal structures of interest here [Fig. 7(a) and (b)] illustrates the anisotropy of the elastic properties of solid ${ }^{4} \mathrm{He}$.

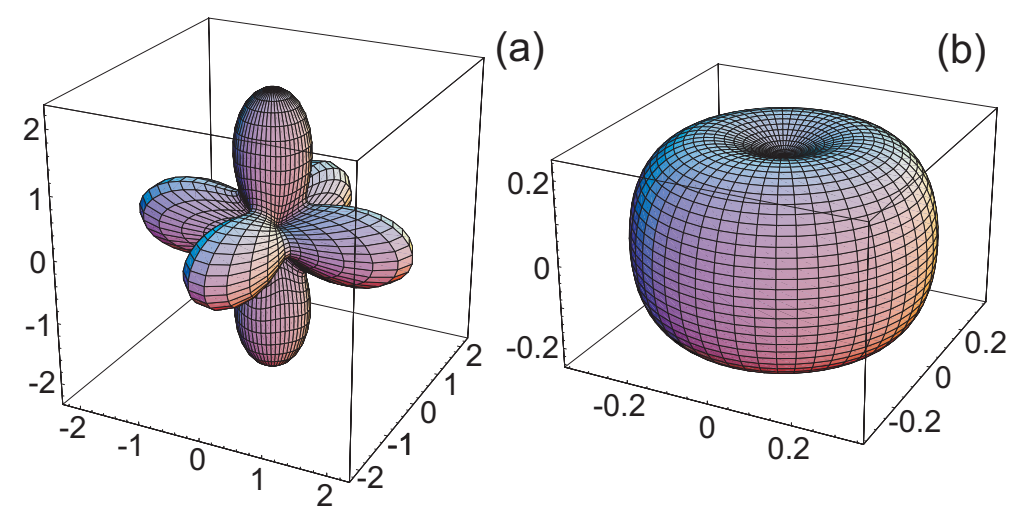

Fig. 7. Angular dependence of $1 / E(\mathbf{n})$ for (a) bcc (1.5 K, 26.6 bar) and (b) hcp (1.5 K, 27 bar) solid helium in units of $10^{7}$ Pa.

The stiffness tensor in hcp crystal reflects well the relative hardness of the crystal along the c-axis compared to the softer (and isotropic) elasticity along the basal plane. For bcc the crystal is harder along the space diagonals compared to deformations perpendicular to the unit cell's faces. We note the different scales in Figs. 7(a) and (b): the hcp crystal is much harder than the bcc crystal for any direction of the applied stress.

The symmetry properties of the stiffness tensor can be better seen by decomposing $\frac{1}{E(\mathbf{n})}$ into multipole moments according to

$$
\left.\frac{1}{E(\mathbf{n})}=\sum_{l=0}^{4} \sum_{m=-l}^{l} a_{l, m} Y^{(} l\right)_{m}(\vartheta, \phi) .
$$

Because of the rank 4 nature of the tensor only multipoles up to $l=4$ contribute to the expansion. For bcc the expansion has the form

$$
\left(\frac{1}{E(\mathbf{n})}\right)_{b c c} \propto Y_{0}^{(0)}(\vartheta, \phi)-a Y_{0}^{(4)}(\vartheta, \phi)-b \operatorname{Re} Y_{4}^{(4)}(\vartheta, \phi),
$$

while for hep one finds

$$
\left(\frac{1}{E(\mathbf{n})}\right)_{h c p} \propto Y_{0}^{(0)}(\vartheta, \phi)-c Y_{0}^{(2)}(\vartheta, \phi)+d Y_{0}^{(4)}(\vartheta, \phi) .
$$

Due to the anisotropy of Young's modulus the elastic force $F_{\text {elastic }}$ exerted on the atomic bubbles has an orientational dependence. The shape of the bubble interface resulting from the equilibrium between the repulsive forces between He atoms and a dopant atom and the elastic restoring forces (Eq. 10) will thus become non-spherical, even if the dopant atom is in a spherically symmetric state. One therefore expects that the atomic bubbles are deformed, both in bcc and in hep crystals. 
Fig. 7 shows that the anisotropy is more pronounced in the cubic phase than in the hexagonal phase. Together with the softer nature of the bcc crystal one might be led to conclude that deformation effects might be more pronounced in bcc than in hcp. However, as the multipole analysis shows the anisotropy of the bcc crystal is given by $l=4$ (hexadecupole) contributions, while the lowest order anisotropy in hcp is given by a $l=2$ (quadrupole) contribution. As we will see below the interaction of the atom with bubble deformations drops rapidly with the increasing multipolarity of the anisotropy. In this sense the bcc matrix may be considered to be an isotropic medium with respect to its perturbation of atomic wavefunctions.

The question whether or not nanometer-scale structures can still be described in a meaningful way by the macroscopic concept of Young's modulus and whether its effects on experimental observations makes sense will be addressed in the following section.

\subsection{Deformed bubbles in hcp phase}

Static atomic bubble deformations in the hcp phase of solid He have been addressed for the first time by Kanorsky et al. [70]. A detailed account of the calculation of atomic bubble shapes formed by a Cs atom in the spherically symmetric $6 S_{1 / 2}$ ground state in the anisotropic hcp solid He will be given in [71]. The interaction between $\mathrm{Cs}$ and $\mathrm{He}$ is modelled using the pair potential $U_{6 S}(R)$ from [50]. The calculation starts with a spherical bubble, following the model described in Sections 3.2 and 3.4 yielding the equilibrium spherical bubble radius $R_{b}$ [48]. The deformation is introduced by giving the parameter $R_{0}$ entering the expression for the He density (Eq. 4) an explicit angular dependence. Since the leading anisotropy term in the multipole expansion of the stiffness matrix in hcp (Eq. 16) is proportional to $Y_{0}^{(2)}$ one can represent $R_{0}$ as

$$
R_{0}(\vartheta)=R_{s p}\left[1+\beta \frac{3 \cos ^{2} \vartheta-1}{2}\right]
$$

The bubble radius parameter $R_{s p}$ defines the average size of the bubble and $\beta$ characterizes the degree of bubble deformation. Note that the angular integration of the second term in (17) vanishes.

The force acting on a surface element $d S(\vartheta, \phi)$ of the bubble interface is calculated as the derivative of the interaction energy between the Cs atom and all He in the line of sight of the surface element

$$
F_{C s-H e}(\vartheta)=\frac{d}{d R_{s p}} \int_{R_{0}(\vartheta)}^{\infty} U_{6 S}(R) \rho(R, \vartheta) R^{2} d R,
$$

and the elastic force is given by

$$
F_{\text {elastic }}=-F_{C s-H e}=F_{\text {spher }}+F(\vartheta) .
$$

The effect of the isotropic elastic force $F_{\text {spher }}$ is discussed in Sec. 3.4, and the bubble deformation is due to the $\vartheta$-dependent force $F(\vartheta)$. This force acts along $\widehat{R}$ and has both normal, $F_{n}$, and tangential, $F_{t}$, components with respect to the local (deformed) interface. The longitudinal and shear stresses are proportional to $F_{n}$ and $F_{t}$ respectively. The strain $\varepsilon$ defined 


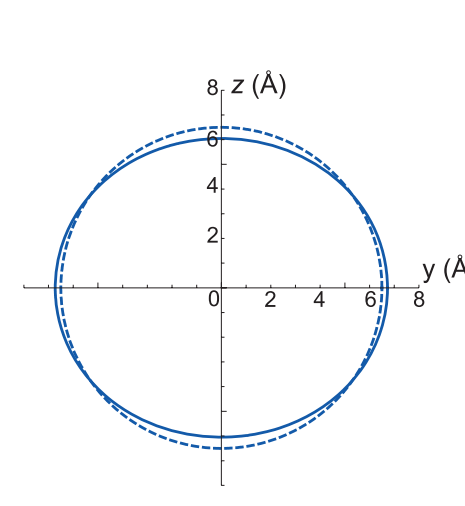

$\operatorname{Cs}\left(6^{2} S_{1 / 2}\right)$ in hcp solid He

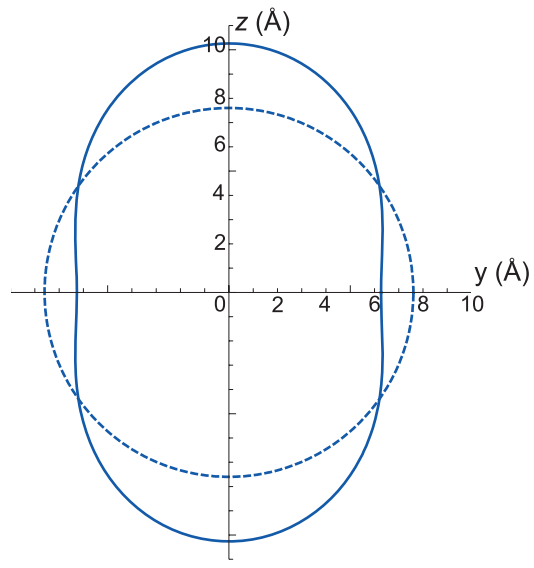

$\mathrm{Ba}\left(6^{1} P_{1}\right)$ in liquid $\mathrm{He}$

Fig. 8. Examples of atomic bubbles with a quadrupole deformation: bubble of a ground-state cesium atom deformed by the elastically anisotropic hcp He matrix (left) and bubble formed by a non-spherical excited Ba atom in liquid He (right). Dashed lines show non-deformed spherical bubbles of the same $R_{0}$.

in Section 4.1 can be calculated from Hooke's law (Eq. 12) in which the numerical values of the elements of the stiffness tensor for hcp are taken from [17]. The change of the bubble radius in the direction $\widehat{R}(\vartheta, \phi)$ is given by the projection of the longitudinal strain onto this direction, followed by an integration over $R$. On the other hand, a change of the radius in that direction is given by $\frac{1}{2} R_{s p} \beta\left(3 \cos ^{2} \vartheta-1\right)$ according to Eq. 17 . From this we find a deformation parameter $\beta=0.07$ after using an iterative procedure [71]. The value is consistent with an earlier experimental determination (see Sec. 9.5 and 9.8). In Fig. 8 we show $R_{0}(\vartheta)$ corresponding to this deformed bubble.

\subsection{Interaction of a Cs atom with a deformed bubble}

If the the bubble shape is known one can apply perturbation theory to find the wavefunction and energies of the atom embedded in the bubble. With this knowledge it should then be possible to infer specific effects of the bubble deformation on the optical transitions and on the intra-/inter-multiplet magnetic resonance transitions.

The Hamiltonian of the valence electron in a deformed bubble atom is given by Eq. 5, modified to give the helium density an explicit $\vartheta$ dependence. The electronic wavefunction $\Psi$ is represented in the basis of unperturbed atomic wavefunctions $\left|n, L, M_{L}, S, M_{S}\right\rangle$ and the interaction with the bubble deformation is treated as a perturbation $V_{\mathrm{bub}}(r, \vartheta, \phi)$. If the deformation has a specific multipolarity, described by an angular dependence $Y_{m}^{(l)}(\vartheta, \phi)$, it is reasonable to assume that the perturbing Hamiltonian has the same symmetry, so that the perturbation Hamiltonian can be written as

$$
\begin{aligned}
V_{\mathrm{bub}}(r, \vartheta, \phi) & =V_{0}(r)\left[Y_{0}^{(0)}+a_{l m} Y_{m}^{(l)}(\vartheta, \phi)\right] \\
& \equiv V_{\mathrm{bub}}^{\mathrm{spher}}(r)+a_{l m} V_{0}(r) Y_{m}^{(l)}(\vartheta, \phi),
\end{aligned}
$$

in which the first term describes the perturbation of the free atom by a spherical bubble and the second term its perturbation by the bubble anisotropy. The angular momentum selection 
rules imply that any perturbation with $l \neq 0$ will mix a given orbital angular momentum state $|L\rangle$ of the atom with states up to $|L+l\rangle$.

In a spherical bubble $(\beta=0)$ the perturbation potential $V_{\text {bub }}=V_{\text {bub }}^{\text {spher }}$ is scalar, i.e., independent of $\vartheta$. It can thus not couple states with different orbital momenta but is capable of mixing $n S_{1 / 2}$ states with different principal quantum numbers $n$. The ground state wavefunction $\widetilde{6 S_{1 / 2}}$ perturbed by a spherical bubble can therefore be expanded in temrs of unperturbed $n S_{1 / 2}$ wavefunctions. By an explicit comparison with the exact solution obtained in [48], we have shown that $99 \%$ of the Cs-He interaction energy of the perturbed ground state can be accounted for by mixing $n S_{1 / 2}$ with $n=7 \ldots 9$ into the $n=6$ ground state.

\subsubsection{Perturbation of the ground state in the hcp phase}

As shown by Eq. 16 the lowest order bubble deformation in hcp has a $l=2$ (quadrupole) character, so that the perturbation operator can be written as

$$
V_{\mathrm{bub}}^{\mathrm{hcp}}(r, \vartheta, \phi)=a_{20} V_{0}(r) Y_{0}^{(2)}(\vartheta, \phi) .
$$

Because of its symmetry $V_{\text {bub }}^{\text {hcp }}$ has the selection rule $\Delta L=0, \pm 2(\Delta L= \pm 1$ is forbidden by parity conservation) which imply that the only non-vanishing matrix elements couple the ground state to excited $n D$ states. Let us first address the effect of $V_{\text {bub }}^{\text {hcp }}$ on the hyperfine Zeeman levels of the $6 S_{1 / 2}$ ground state of Cs.

The selection rule $\Delta L=2$ implies that hexagonal bubbles do not affect the ground state energy in first order. The second order perturbation yields a global lowering of the ground state energy levels which does not depend on $F$, nor on $M$. Using unperturbed level energies and considering only the contribution from the lowest lying $5 D$ states the second order shift of the ground state energy is

$$
\Delta E^{(2)}=-87.3 \varepsilon_{6 S, 5 D}^{2} \mathrm{THz}=-2912 \varepsilon_{6 S, 5 D}^{2} \mathrm{~cm}^{-1},
$$

where the $S-D$ mixing coefficient $\varepsilon_{6 S, n D}$ is given by

$$
\varepsilon_{6 S, n D}=\frac{\left\langle n D\left\|V_{\mathrm{bub}}^{\mathrm{hcp}}\right\| 6 S\right\rangle}{E_{6 S}-E_{n D}} .
$$

Since $\varepsilon_{6 S, 5 D}^{2} \approx 10^{-3}$ (see Sec. 9.8 below) the bubble deformation-induced shift of the ground state is approximately $-3 \mathrm{~cm}^{-1}$.

It is interesting to note the similarity of the present calculation with the calculation of the Stark effect in second and third order [72], in which the perturbation operator has the structure $V_{\mathrm{St}} \propto z \propto f(r) Y_{0}^{(1)}$, and in which the second order perturbation of the $6 S_{1 / 2}$ state also gives an $F$ and $M$ independent energy shift.

From the perturbative treatment of the Stark effect it is known that third order perturbation theory which treats the Stark and hyperfine interactions at an equal level yields $F$ - and $M$ - dependent energy shifts of the ground state levels. We may apply the general expression of the third order energy perturbation

$$
\Delta E^{(3)}(\alpha)=\sum_{\beta \neq \alpha, \gamma \neq \alpha} \frac{\langle\alpha|W| \beta\rangle\langle\beta|W| \gamma\rangle\langle\gamma|W| \alpha\rangle}{\left(E_{\alpha}-E_{\beta}\right)\left(E_{\alpha}-E_{\gamma}\right)}-\langle\alpha|W| \alpha\rangle \sum_{\beta \neq \alpha} \frac{|\langle\beta|W| \alpha\rangle|^{2}}{\left(E_{\alpha}-E_{\beta}\right)^{2}},
$$




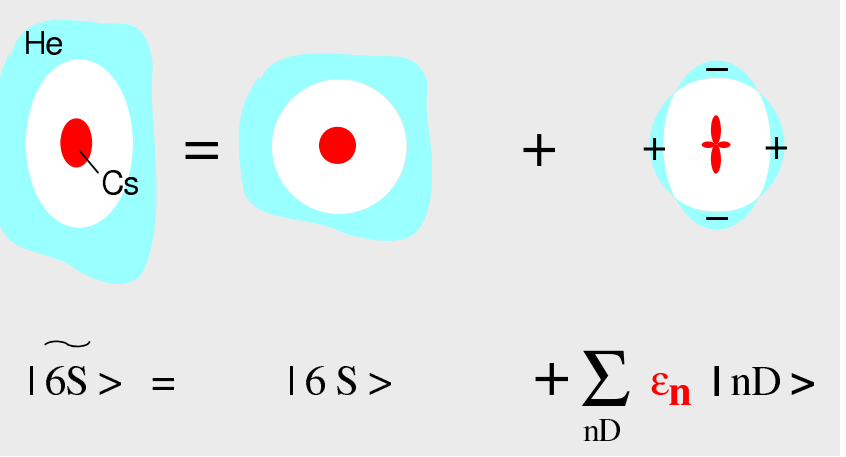

Fig. 9. Effect of a deformed bubble - represented as a spherical bubble plus a quadrupolar excess/defect mass distribution - on a spherically symmetric alkali atom. The deformation admixes fractions of $D$-orbitals into the ground state's $S$-orbital thereby producing an electronic density distribution which reflects the bubble shape.

of the ground state $|\alpha\rangle=\left|6 S_{1 / 2}, F, M\right\rangle$ energies to the present problem by setting $W=$ $V_{\mathrm{bub}}^{\mathrm{h} p \mathrm{p}}+H_{\mathrm{hfs}}$.

The energy shifts resulting from the second term of Eq. 25 do not depend on $M$, but they do yield a red shift of the hyperfine transition frequency

$$
\begin{aligned}
\Delta E_{h f}^{\text {def.bub. }}\left(6 S_{1 / 2}\right) & =\Delta E^{(3)}\left(6 S_{1 / 2}, 4, M\right)-\Delta E^{(3)}\left(6 S_{1 / 2}, 3, M\right) \\
& =-\frac{4}{5} A_{\mathrm{hf}}\left(6 S_{1 / 2}\right) \sum_{n D} \varepsilon_{6 S, n D}^{2} .
\end{aligned}
$$

In Sec. 9.5 we will use the last result for an analysis of the change of the hyperfine frequency at the bcc-hcp phase boundary. The analysis of this shift has allowed the authors of [70] to infer a $6 S-5 D$ mixing coefficient of $3 \%$ and from that a bubble deformation parameter of $\beta=6-7 \%$, that was later confirmed by the calculation [71] based on the elastic anisotropy of solid He.

After applying the Wigner-Eckart theorem, angular momentum decoupling rules and algebraic summations, the first term of Eq.(25) reduces to

$$
\begin{aligned}
\Delta E^{(3)}\left(6 S_{1 / 2}, 3, M\right)= & \sum_{n D_{J}} \frac{18 A_{\mathrm{hf}}\left(n D_{3 / 2}\right)-63 A_{\mathrm{hf}}\left(n D_{5 / 2}\right)}{100} \varepsilon_{6 S, n D}^{2} \\
& +\sum_{n D_{J}} \frac{A_{\mathrm{hf}}\left(n D_{5 / 2}\right)-A_{\mathrm{hf}}\left(n D_{3 / 2}\right)}{100} \varepsilon_{6 S, n D}^{2}\left(3 M^{2}-12\right),
\end{aligned}
$$

and

$$
\begin{aligned}
\Delta E^{(3)}\left(6 S_{1 / 2}, 4, M\right)= & \sum_{n D_{J}} \frac{-14 \mathrm{~A}_{\mathrm{hf}}\left(\mathrm{nD}_{3 / 2}\right)+49 \mathrm{~A}_{\mathrm{hf}}\left(\mathrm{nD}_{5 / 2}\right)}{100} \varepsilon_{6 S, n D}^{2} \\
& -\sum_{n D_{J}} \frac{\mathrm{A}_{\mathrm{hf}}\left(\mathrm{nD}_{5 / 2}\right)-\mathrm{A}_{\mathrm{hf}}\left(\mathrm{nD}_{3 / 2}\right)}{100} \varepsilon_{6 S, n D}^{2}\left(3 M^{2}-20\right),
\end{aligned}
$$

The explicit $M$-dependence of the latter expressions makes that these contributions shift the magnetic resonance transition frequencies between Zeeman sublevels. This effect may even 
lift the Zeeman degeneracy (crystal field splitting) in the absence of an applied external magnetic field. With $\mathrm{Cs}$ in hcp ${ }^{4} \mathrm{He}$ this splitting is large enough $(10 \mathrm{kHz})$ to be measured, an effect that will be analyzed in Sec. 9.8.

\subsubsection{Effects of deformed bubbles in hcp on excited states}

For the detailed analysis of the optical absorption spectra presented in Sec. 7.3 it is important to know the perturbation of the excited states by its interaction with a deformed bubble. Because of the lower symmetry of the excited $6 P_{1 / 2}$ and $6 P_{3 / 2}$ states, compared to the highly symmetric ground $6 S_{1 / 2}$ state the deformed bubble Hamiltonian $V_{\text {bub }}^{\text {hcp lifts }}$ the $M_{J}$ degeneracy of the $6 P_{3 / 2}$ sublevels already in first order perturbation theory via $E^{(1)}\left(6 P_{3 / 2}, M_{J}\right)=\left\langle 6 P_{3 / 2}, M_{J}\left|V_{b u b}\right| 6 P_{3 / 2}, M_{J}\right\rangle$. As a consequence the state splits into two doubly-degenerated components with $M_{J}= \pm 1 / 2$ and $M_{J}= \pm 3 / 2$, corresponding to an alignment of the dumbbell-shaped $P$-orbital either along or across the deformed bubble. This effect leads to a splitting of the $D_{2}$ absorption line (Fig. 19(b)). In second order, the perturbation mixes $M_{J}= \pm 1 / 2$ sublevels belonging to different $n P_{J}$ states. In Cs the largest contribution is due to $6 \mathrm{P}_{1 / 2}-6 P_{3 / 2}$ mixing given by

$$
E^{(2)}\left(6 P_{J=L \pm 1 / 2}, M\right)= \pm \frac{\left|\left\langle 6 P_{3 / 2}, M\left|V_{\mathrm{bub}}^{\mathrm{hcp}}\right| 6 P_{1 / 2}, M\right\rangle\right|^{2}}{\Delta E_{F S}(6 P)}
$$

where $\Delta E_{F} S(6 P)=554 \mathrm{~cm}^{-1}$ is the fine-structure splitting. The unperturbed $6 P_{1 / 2}$ orbital is spherically symmetric. The admixture of the $6 P_{3 / 2}$ state deforms it into an apple-shaped orbital that fits better into the deformed bubble. Therefore both $M_{J}$ components of the $6 P_{1 / 2}$ state are shifted to lower energies and the corresponding absorption line shifts to the red, as observed in experiments [48] at the bcc-hcp phase transition. The same effect shifts the $M_{J}= \pm 1 / 2$ component of the $6 P_{3 / 2}$ state to higher energies thus increasing the (first order) splitting of the $D_{2}$ absorption line.

It is interesting to note that a static quadrupolar deformation as described by Eq. 17 was considered in [65] for electron bubbles in superfluid He, trapped at vortices and experiencing an anisotropic Bernoulli pressure. The deformation is rather small $(\beta=0.023)$ and the resulting splitting of the $M_{L}$ components of the $1 p$ state is about $30 \mathrm{~cm}^{-1}$, much smaller than the $1 s-1 p$ transition linewidth.

\subsubsection{Perturbation of the ground state in the bcc phase}

Because of the elastic anisotropy discussed above (Sec.4.1) the shape of the bubbles in bcc ${ }^{4} \mathrm{He}$ has a contribution with a $l=4$ (hexadecupole) symmetry, so that the perturbation operator acquires a contribution with the same symmetry

$$
V_{\mathrm{bub}}^{\mathrm{bcc}}(r, \vartheta, \phi)=V_{0}(r)\left[a_{40} Y_{0}^{(4)}(\vartheta, \phi)+a_{44} \operatorname{Re} Y_{4}^{(4)}(\vartheta, \phi)\right] .
$$

This interaction will mix atomic states with different orbital momenta $L$. Angular momentum and parity conservation imply that $n S$ states will be mixed with $n^{\prime} G$ states, while $n P$ states will be mixed with $n^{\prime} F$ and $n^{\prime \prime} H$ states $(P-G$-mixing being forbidden by parity conservation). 
Because of the $\Delta L \neq 0$ selection rule the perturbation will produce no first order effect. In second order perturbation theory the deformed bubble will give a common shift to all ground state hyperfine Zeeman levels on the order of

$$
\Delta E^{(2)} \approx \frac{\left\langle n G\left|V_{\mathrm{bub}}^{\mathrm{bcc}}\right| 6 S\right\rangle}{\Delta E_{6 S, n G}},
$$

based on similar arguments as presented in Sec. 4.3.1 for bubbles in hcp crystals. From the same arguments one expects that the interaction (Eq. 31) yields $F$ - and $M$-dependent level shifts in third order. The $F$-dependent level shifts are suppressed with respect to Eq. 32 by $\Delta E_{h f s}(6 S) / \Delta E_{6 S, n G}$, where the energies refer to the hyperfine structure and optical transition frequencies, respectively. If present, such an effect may be inferred from the pressure dependence of the $6 S(F=4, M=0)-6 S(F=3, M=0)$ clock transition frequency near the liquid-bcc phase transition. A clear signature would be a jump of the transition frequency (plotted versus He density) that cannot be accounted for by spherical bubble model predictions. A quantitative statement about such an effect is not possible with present knowledge. Even though the pressure dependence of the hyperfine transition frequency in bcc has been explored experimentally [73], it has only been observed under conditions of saturated vapor pressure in the liquid phase [74]. On the other hand one expects the $M$-dependent effects that appear in third order to be suppressed by $\Delta E_{h f s}(n G) / \Delta E_{6 S, n G}$ with respect to Eq. 32, which will be much smaller than the corresponding effect $(10 \mathrm{kHz})$ in hcp ${ }^{4} \mathrm{He}$ (cf Sec. 9.8) because of the smallness of the hyperfine splitting in the $n G$ states and their large excitation energy.

\subsection{Bubbles of non-spherical electronic states}

The shape of the bubble formed around an excited state of an electron in liquid He was analyzed in [75-79]. A simplified bubble model was applied that assumed a sharp bubble interface and neglected the penetration of the electron wavefunction into the helium as well as its polarization energy $V_{\text {pol }}(\mathbf{r}, \mathbf{R})$. The angular dependence of the bubble radius was represented by a superposition of axially symmetric spherical harmonics $Y_{0}^{(l)}$, adjusted such as to minimize the total defect energy. The equilibrium bubbles formed by the excited $1 p$ and $2 p$ states were found to be dumbbell-shaped. With increasing He pressure the diameter of the dumbbell's neck decreases and vanishes almost completely above 10 bar. This result led the author of [75] to speculate whether a fission of the excited electron bubble might occur, yielding an interesting quantum object described by a delocalized split wavefunction, each part of which would be contained in a localized bubble.

This hypothesis was criticized in a number of works [80-83] that insisted on the indivisibility of the electron. As suggested in [82], the relaxation of the excited-state bubble towards the dumbbell-like configuration results in nearly degenerate $1 s$ and $1 p$ states and in the excitation of an anti-symmetric bubble oscillation mode featuring a synchronous shrinking of one half and an expansion of the other half. The adiabatic (Born-Oppenheimer) approximation becomes invalid when the characteristic tunneling time between the two bubbles becomes comparable to the period of the bubble oscillation. Under those conditions the probability of a radiationless $1 p \rightarrow 1 s$ transition becomes very high and the system returns to the ground 
state on a time scale shorter than the fission time. Another line of argumentation [81] considered the split electron as a coherent delocalized superposition state as stated above with equal probabilities to be found in either half of the bubble. In that case the interaction with the vibrating bubble interface leads to a fast decoherence and the superposition state collapses to a localized state. More recently the $1 p$ and $1 d$ electron bubbles were modeled using the density functional method [84] that provides a more realistic description of the bubble interface than the simplified model of [75]. Those calculations show that the waist of the dumbbell-shaped $1 p$ bubble has a finite diameter and that a splitting of the bubble in two does not occur. The $1 s$ state in the deformed excited state bubble is shifted to a higher energy which still lies $290 \mathrm{~cm}^{-1}$ below the $1 p$ state.

The problem of non-spherical atomic bubbles was addressed in several papers $[57,47,54]$ in which fluorescence spectra from excited atomic states with non-spherical orbitals were calculated. For the $6 P_{3 / 2}$ state of $\mathrm{Cs}$ and the $3 P$ state of $\mathrm{Na}$ [47] a strong deformation of the bubble and a large increase of the He density in the regions of reduced electron density of the dopant (two dimples of the apple-shaped $6 P_{3 / 2}$ orbitals and a nodal plane of the dumbbell-shaped $3 P_{z}$ orbital) were obtained. Both effects were interpreted as signatures of $\mathrm{Cs}^{*} \mathrm{He}_{2}$ and $\mathrm{Na}^{*} \mathrm{He}_{4}$ exciplexes that will be discussed in detail in Sec. 5 and 7.4.

The bubble formed around an excited $\mathrm{Ba}$ atom in the ${ }^{1} P_{1}$ state was modeled in [57] assuming an angular dependence of the bubble radius as given by Eq. 17. The best fit to the observed dependence of the fluorescence wavelength on He pressure was achieved with a parameter $\beta=0.35$, corresponding to a strongly deformed bubble shown in Fig. 8 .

For the ${ }^{1} P_{1}$ excited state of $\mathrm{Mg}$ both the $\mathrm{Mg}^{*} \mathrm{He}_{10}$ exciplex [85] and a deformed bubble [54] have been considered. Different bubble shapes were proposed [54] with quadrupolar (Eq. 17, $\beta=0.413)$ and higher order deformations. In that case the total energy of the defect is minimized for a bubble radius given by

$$
R_{0}(\vartheta)=R_{s p}\left[1+\beta\left(1-\frac{315}{128} \cos ^{8} \vartheta\right)\right]
$$

\subsection{Dynamic bubble deformations}

Vibrations of the bubble interface were already considered in the early studies $[86,37,87]$ of electron bubbles in liquid helium, where the electron's energy was modeled by a simple square well potential. Spherical bubbles subjected to breathing and quadrupole distortions were considered as well. More recently $[61,62]$, bubble shape oscillations described by $Y_{0}^{(l)}$ and $Y_{m}^{(l)} \pm Y_{m}^{(l) *}$ type deformations with $l=0,2,3$ were analyzed at different temperatures and He pressures. The calculations show that all types of vibrations oscillate at frequencies on the order of $10^{10} \mathrm{~Hz}$ that increase with helium pressure.

The authors of $[61,62]$ have modeled the $1 s \rightarrow 1 p$ transition lineshapes using the method described in Sec. 3.3. Good agreement with the experimental results of [65] was achieved when breathing and quadrupole oscillation modes were taken into account.

Quadrupolar oscillations lift the degeneracy of the $M=0$ and $M= \pm 1$ levels of the $1 p$ excited state (Jahn-Teller effect) [37]. However, due to the very large spectral width of the $1 s \rightarrow 1 p$ transition such a splitting was never observed experimentally. 
Atomic absorption lines in condensed helium, on the other hand, have been studied with a better spectral resolution and have allowed a comparison between calculated and measured lineshapes. Quadrupolar atomic bubble oscillations were first considered in $[46,47]$ for explaining the characteristic doubly-shaped contour of the $n S_{1 / 2} \rightarrow n P_{3 / 2}$ excitation line of $\mathrm{Rb}$ and $\mathrm{Cs}$ in superfluid He. The splitting of the excited $n P_{3 / 2}$ states is due to the same mechanism as discussed in Sec. 4.4 for excited Cs atom in bubbles with a static quadrupole deformation. In the case of dynamic bubble deformations the perturbation is time-dependent and the effect has to be averaged over the oscillation period (in an adiabatic approximation). We will come back to this point in Sec. 11.2.

A more general approach including monopole (breathing mode), dipole, and quadrupole modes of bubble oscillations was applied in $[58,56]$ for the analysis of the excitation spectra of $\mathrm{Yb}^{+}$ions and $\mathrm{Ca}$ atoms in liquid helium. For $\mathrm{Yb}^{+}[58]$ the situation is very simlar to that of alkali atoms. Both dipole and quadrupole types of vibrations split the $4 f^{14} 6 p$ ${ }^{2} P_{3 / 2}$ state into two components and do not affect the $4 f^{14} 6 p^{2} P_{1 / 2}$ state. The splitting by the quadrupole vibrations is much larger and dominates over the splitting of the $D_{2}$ line absorption spectrum. For Ca, the authors of [56] claim that the $4 s 4 p{ }^{1} P_{1}$ state is split by the dipole oscillation mode into two components and by the quadrupole mode into three components. The latter statement seems to be erroneous, since the quadrupole perturbation can not split the $M_{L}= \pm 1$ sublevels. All other studied excited states of Ca, the metastable $4 s 4 p{ }^{3} P_{0}$ and the $4 s 5 s{ }^{3} S_{1}$ state are spherically symmetric and are therefore not split by the bubble deformations. The splitting of the ${ }^{1} P_{1}$ state of $\mathrm{Ca}$ is much smaller than in $\mathrm{Yb}^{+}$ and in the alkalis and cannot be resolved. The resulting spectral profile of the ${ }^{1} S_{0} \rightarrow{ }^{1} P_{1}$ absorption line is strongly asymmetric, in contrast to that of the ${ }^{3} P_{0} \rightarrow{ }^{3} S_{1}$ transition. For both elements the experimental absorption spectra $[58,56]$ were found to agree well with the calculated ones.

The interaction with the vibrating bubble can also lead to a lifting of selection rules and to an enhancement of forbidden transitions. This effect was studied theoretically for the $1 s \rightarrow 1 d$ transition in the electron bubble in liquid He [88].

\subsection{Molecular bubbles}

Small molecules are also known to form microscopic bubbles in condensed He. Already in early studies [89] of $\mathrm{He}_{2}^{*}$ excimers in liquid $\mathrm{He}$ it was suggested that such molecules reside in bubbles, similar to the bubbles formed by excited $\mathrm{He}^{*}$ atoms.

First calculations of molecular bubble were reported for the hydrogen molecule [90] and used to interpret the observed $e^{3} \Sigma_{u} \rightarrow a^{3} \Sigma_{g}$ fluorescence (see Sec. 7.6). A spherical shape was assumed for bubbles formed by both molecular states. The interaction between the valence electron of the molecule and surrounding helium was modeled by a pseudopotential and the radial Schrödinger equation was solved as discussed in Sec. 3.2. As a result, the spectral shift of the fluorescence could be predicted with sufficient accuracy, although the spectral width was underestimated. Density functional calculations of the bubble formed by $\mathrm{H}_{2}$ in liquid He were presented more recently [91]. The calculations [91] have shown a strongly anisotropic $\mathrm{H}_{2}$-helium interaction that favors exciplex formation in the $e^{3} \Sigma_{u}$ state. Only for a freely rotating molecule the spherically averaged interaction results in the formation of a 

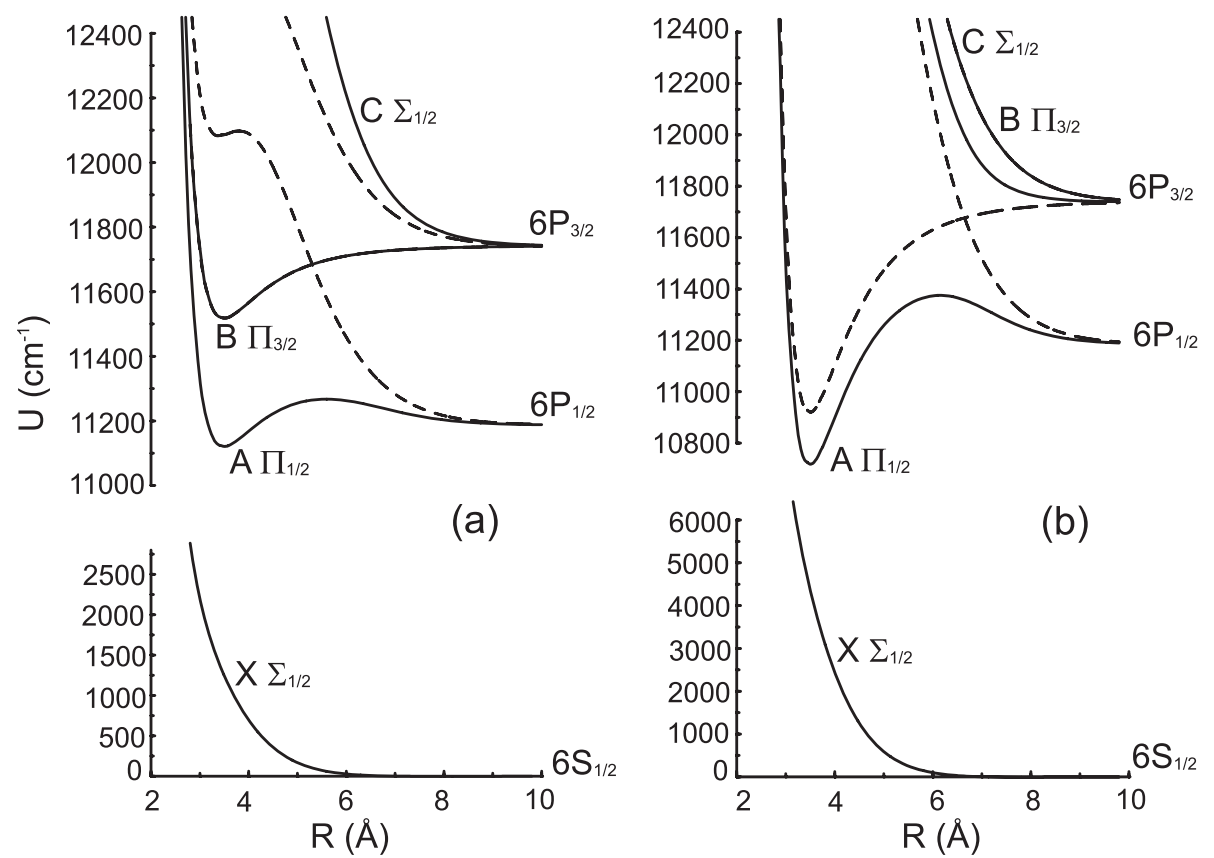

Fig. 10. Potential energy diagrams for $\mathrm{Cs}^{*} \mathrm{He}_{2}$ (a) and $\mathrm{Cs}^{*} \mathrm{He}_{7}$ (b) exciplexes. Solid lines - adiabatic potential curves, dashed lines - diabatic potential curves.

bubble with a radius of $\approx 10 \AA$, in agreement with the model of [90].

Density functional calculations were also reported for triplet states of $\mathrm{He}_{2}^{*}$ excimers in liquid He [92]. For the nearly spherically symmetric metastable $a^{3} \Sigma_{u}$ state a bubble with a radius of $7 \AA$ was obtained. For the $b^{3} \Pi_{g}$ and $c^{3} \Sigma_{g}$ states whose orbitals posses nodal planes a transformation into $\mathrm{He}_{3}^{*}$ or larger exciplexes is predicted. In the case of the $c^{3} \Sigma_{g}$ state this process is suppressed by the rotation of the excimer: the spherically averaged interaction results in a bubble formation. Experimental observations of triplet $\mathrm{He}_{2}^{*}$ absorption and fluorescence (see Sec. 7.5) can be explained by this model.

\section{Alkali-helium exciplexes}

The term "exciplex" stands for excited state complex and refers to molecular complexes which form bound or quasibound states only when one of their constituents is in an excited state. Exciplexes composed of an alkali atom in a $n P$ state and of one or several ground state He atoms were considered for the first time in [51,93] to explain the quenching of the laserinduced fluorescence from light alkalis in liquid and solid helium. It was also shown in [94] that the quenching of $\mathrm{Rb}$ fluorescence in pressurized liquid He is due to exciplex formation, a process which becomes the dominant deexcitation channel for excited $\mathrm{Rb}$ atoms at high He pressures.

First theoretical treatments of alkali-helium exciplexes [94-99] were based on pair potentials calculated by Pascale [50]. Ab initio pair potentials were also used to model $\mathrm{Ag}^{*} \mathrm{He}$ $[100,101]$ and $\mathrm{Ba}^{+*} \mathrm{He}$ excimers $[102,103]$. A number of calculations using modern computational methods of quantum chemistry were applied later to alkali atoms [104-109] and to silver $[110,111]$. 
The theoretical model for describing the optical properties of exciplexes developed in $[112,68]$ is based on adiabatic alkali-helium pair potentials [50], which are strongly anisotropic for the $n P$ states of the alkalis. The alkali-helium interaction at intermediate interatomic distances is dominated by the Pauli repulsion between their valence electrons. When a He atom approaches from a direction along which the electronic density of the alkali orbital is high, it experiences a strong repulsion. However, the $n P$ orbitals possess nodal planes, or nodal axes, along which the electronic density vanishes so that the helium atom can come close enough to experience a van der Waals attraction by the alkali's core. In this case a short-lived weakly bound or quasibound complex can be formed. The ratio of the spin-orbit interaction potential and the (impurity) atom-helium interaction is the relevant parameter that characterizes exciplex formation. For light alkalis the spin-orbit interaction is much weaker than the impurity-He interaction, so that the impurity's excited state orbital is well represented by a dumbbell-shaped $P_{z}$ wavefunction which allows several He atoms to be bound around its waist. For the heavier alkalis, Cs, and to a somewhat lesser extent, Rb, the spin-orbit interaction (which splits the $n P$ state into $n P_{1 / 2}$ and $n P_{3 / 2}$ fine-structure components) becomes comparable to (in the case of $\mathrm{Rb}$ ) or larger than (in the case of $\mathrm{Cs}$ ) the impurity-helium interaction and the symmetry of the state is determined by its total angular momentum $J$.

The $n P_{1 / 2}$ state is spherically symmetric [Fig. 11(d) and (f)] and is hence repulsive for He. The $n P_{3 / 2}$ state, on the other hand, has two distinct orbitals depending on the projection $\left|M_{J}\right|$ of of its angular momentum $J$ on the internuclear axis. The approaching He atom sees either a repulsive dumbbell-shaped orbital oriented along the direction of approach in the $M_{J}= \pm 1 / 2$ configuration [Fig. 11(b)], or an attractive apple-shaped orbital $\left(M_{J}= \pm 3 / 2\right.$ configuration) with two dimples on opposite sides, again along the direction of approach [Fig. 11(a)] [93]. The corresponding potentials are shown in Fig. 10(a), where it is assumed that the two He atoms approach the Cs atom simultaneously along the common axis. A potential well in the potential curve correlating asymptotically to the $6 P_{1 / 2}$ state appears due to the mutual perturbation of the $M_{J}= \pm 1 / 2$ components of the $6 P_{1 / 2}$ and $6 P_{3 / 2}$ states. The atomic orbital of $\mathrm{Cs}$ in this case changes its shape from spherical at large $\mathrm{Cs}-\mathrm{He}$ separations [Fig. 11(d)] to apple-shaped at 3.5 $\AA$ [Fig. 11(c)]. The effect is similar to the one observed in the hcp matrix, in which the atomic bubbles have a quadrupolar deformation (see Sec. 4.3). In both cases the perturbation due to the interaction with He has the same symmetry. The corresponding diabatic potential curves, obtained by neglecting the mixing of the two states are indicated by dashed lines in Fig. 10(a) .

The fine structure mixing becomes even more pronounced in a complex composed of several He atoms arranged on a circle around the alkali atom. If the radius of the He-ring is small enough, the alkali-He interaction becomes comparable to the spin-orbit interaction in the alkali atom and a spin-orbit uncoupling occurs. The corresponding potential energy diagram is shown in Fig. 10(b), where it is assumed that 7 He atoms are uniformly distributed on a ring of radius $\mathrm{R}$ with the $\mathrm{Cs}$ atom in the center. There is an anticrossing between the two potential curves correlating to the $M_{J}= \pm 1 / 2$ components of the $6 P_{1 / 2}$ and $6 P_{3 / 2}$ states. The corresponding diabatic curves (dashed lines in Fig. $10(\mathrm{~b})$ ) cross at $\mathrm{R}=6.6 \AA$. To the left of this point the lower state undergoes a complete shape transformation into a dumbbell-like $P_{z}$ orbital [Fig. 11(e)]. In this case a polyatomic $\mathrm{Cs}^{*} \mathrm{He}_{N}$ exciplex can be formed, analogous 

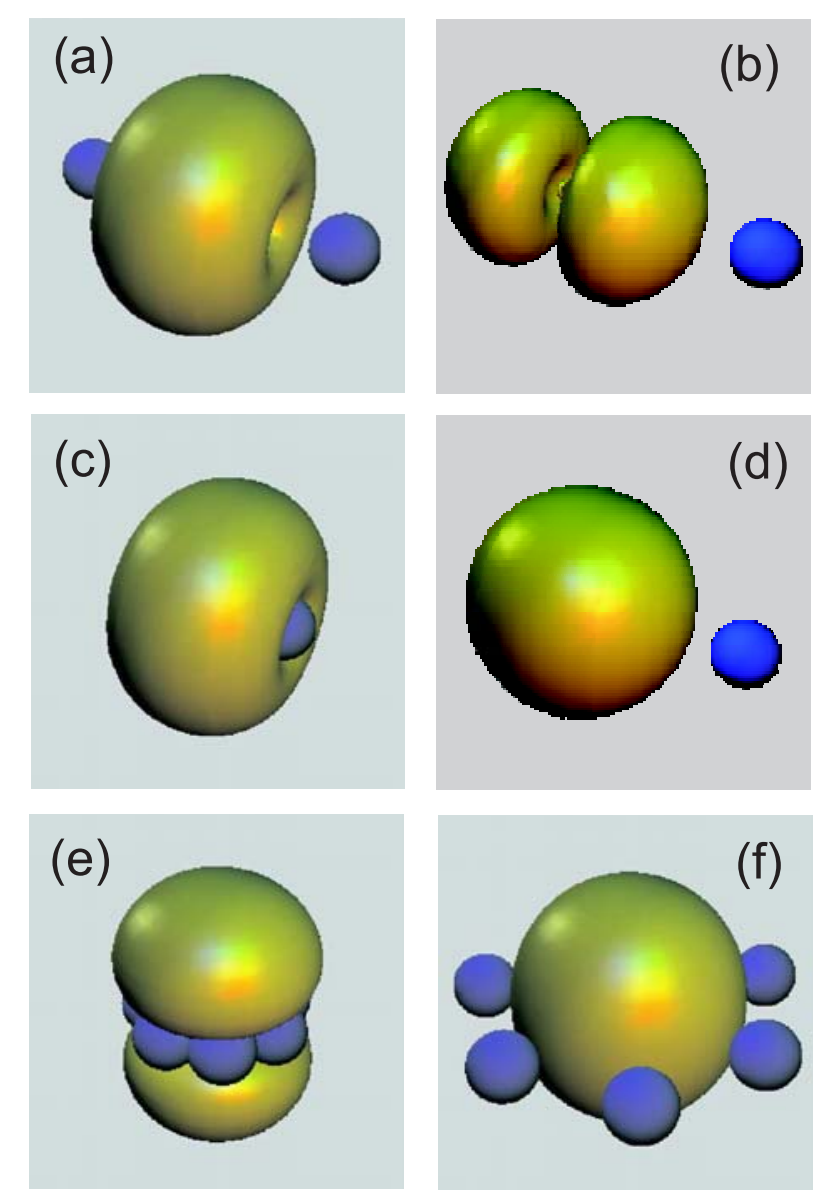

Fig. 11. Structure of $\mathrm{Cs}^{*} \mathrm{He}_{N}$ exciplexes. The valence electron density of Cs atom is shown in yellow and that of He in blue. (a) $\mathrm{B}_{3 / 2}$ state of $\mathrm{Cs}^{*} \mathrm{He}_{2}$; (b) - $\mathrm{C}_{1 / 2}$ state of $\mathrm{Cs}^{*} \mathrm{He}_{2}$; (c), (d) - $\mathrm{A}_{1 / 2}$ state of $\mathrm{Cs}^{*} \mathrm{He}_{2}$; (e), (f) - $\mathrm{A}_{1 / 2}$ state of $\mathrm{Cs}^{*} \mathrm{He}_{7}$. In (a), (b), (d), and (f) Cs-He separation $\mathrm{R}=6.5 \AA$; in (c) and (e) $\mathrm{R}=3.5 \AA$.

to the case of light alkali atoms. The number $N$ of He atoms bound around the waist of the dumbbell-shaped orbital is limited by the repulsive He-He interaction. Based on our model, the maximal value of $N$ can be estimated to be 6 or 7 . The same model applied to the $\mathrm{Rb}^{*} \mathrm{He}_{N}$ exciplexes predicts similar exciplex structures with a maximal number of 6 bound He atoms [113]. Alternative theoretical studies of $\mathrm{Cs}^{*} \mathrm{He}_{N}$ [108], $\mathrm{Rb}^{*} \mathrm{He}_{N}$ [104] and the closely related $\mathrm{K}^{*} \mathrm{He}_{N}$ [106] suggest $N=6$ to be the most probable number of bound He atoms.

\section{Experimental setup}

\subsection{Sample preparation}

Unlike all other rare gases, He does not solidify without external pressure. This makes it impossible to prepare the doped sample by the standard deposition method used in matrix isolation spectroscopy with heavier rare gases, in which a gaseous mixture of the host and impurity atoms/molecules/radicals is condensed onto a cold substrate. Implantation techniques for immersing positive impurity ions into liquid He were reported in [114-116], where 
a dc electric field was used to control the drift of ions produced in a glow discharge right above the surface of liquid He into the working volume. The modification of this technique has allowed the authors of [117] to also implant neutral impurities into liquid He. In that experiment alkaline-earth ions were implanted into liquid helium and then neutralized by the recombination with free electrons introduced into the active volume by field-emission from a negatively charged tip located in the helium bath. This experimental technique was successfully applied to dope liquid He with a large number of different atomic impurities (listed in Table 1). The recombination process populates a variety of excited states in the impurity atoms, whose fluorescence was then detected [118]. The initial vaporization of the dopant was achieved either by heating the sample in a special oven [117], or by laser ablation from the target located above the liquid helium level $[43,119,120,118,53]$. It was shown later [121] that laser ablation from a target immersed directly in the He bath produces a significant amount of neutral impurities which can be observed via their laser-induced fluorescence. An important advantage of the latter method is its applicability to pressurized liquid and even solid He. It has been applied by several groups investigating alkali $[122,123,44,74,45,94]$ and alkaline-earth elements [124,125,57,85,56], as well as Ag [100], Al, Yb, Ga, and In [124]. Since the laser ablation in liquid He produces mostly impurity clusters, [124,121,58,56] a second delayed laser pulse was applied in some experiments in order to dissociate the clusters and to produce individual impurity atoms. In other experiments $[122,123,44,74,45,94]$ the same effect was achieved by increasing the repetition rate of the ablation laser up to $1 \mathrm{kHz}$.

The same laser-ablation technique was used to dope solid He first with barium [67] and later with alkali [126] atoms. In the following we give a detailed description of the experimental setup developed by our group $[67,126]$, which was improved over the years and used by us in our studies of $\mathrm{Cs}$ and $\mathrm{Rb}$ atoms in solid He reviewed in this paper.

The He crystal is grown in a cubic pressure cell made of $1.7 \mathrm{~cm}$ thick copper with inner dimensions of $6 \times 6 \times 6 \mathrm{~cm}^{3}$. The cell has five optical windows, one on each side and one on top of the cell. It is immersed in a liquid helium bath contained in a specially designed cryostat with optical windows that are coaxial with the cell windows (Fig. 12). Before the experiment, the cryostat is filled with 80 liters of liquid He at $4.2 \mathrm{~K}$ and then cooled to $1.5 \mathrm{~K}$ by pumping on the liquid He. The holding time of the cryostat depends on the heat load (absorption of laser radiation, high voltage leakage currents, heat conduction via electrical connections to the cell volume, etc.). It typically allows us to perform continuous measurements during 2-4 days. After filling the bath, helium gas is admitted into the pressure cell via a liquid nitrogen cold trap that removes condensable gases. This transfer goes via a $2 \mathrm{~mm}$ diameter thick steel capillary that minimizes heat exchange. The helium used to grow the matrix comes from a 200 bar storage bottle (purity 99.9999\%) connected to the capillary via a buffer volume, which allows the control of the helium pressure in the cell by a needle valve. The temperature is measured by germanium resistors located in the pressure cell and in the He bath respectively.

After reaching the working temperature of $1.5 \mathrm{~K}$, a He crystal is produced by increasing the pressure in the cell to a value above the solidification pressure, typically to $\approx 29.5$ bar.

Alkali atoms are implanted into the He crystal by laser ablation from the target, a cut glass ampule containing $0.5 \mathrm{~g}$ of the metal under investigation, installed under an inert gas atmosphere at the bottom of the pressure cell in the preparation process. The successive 


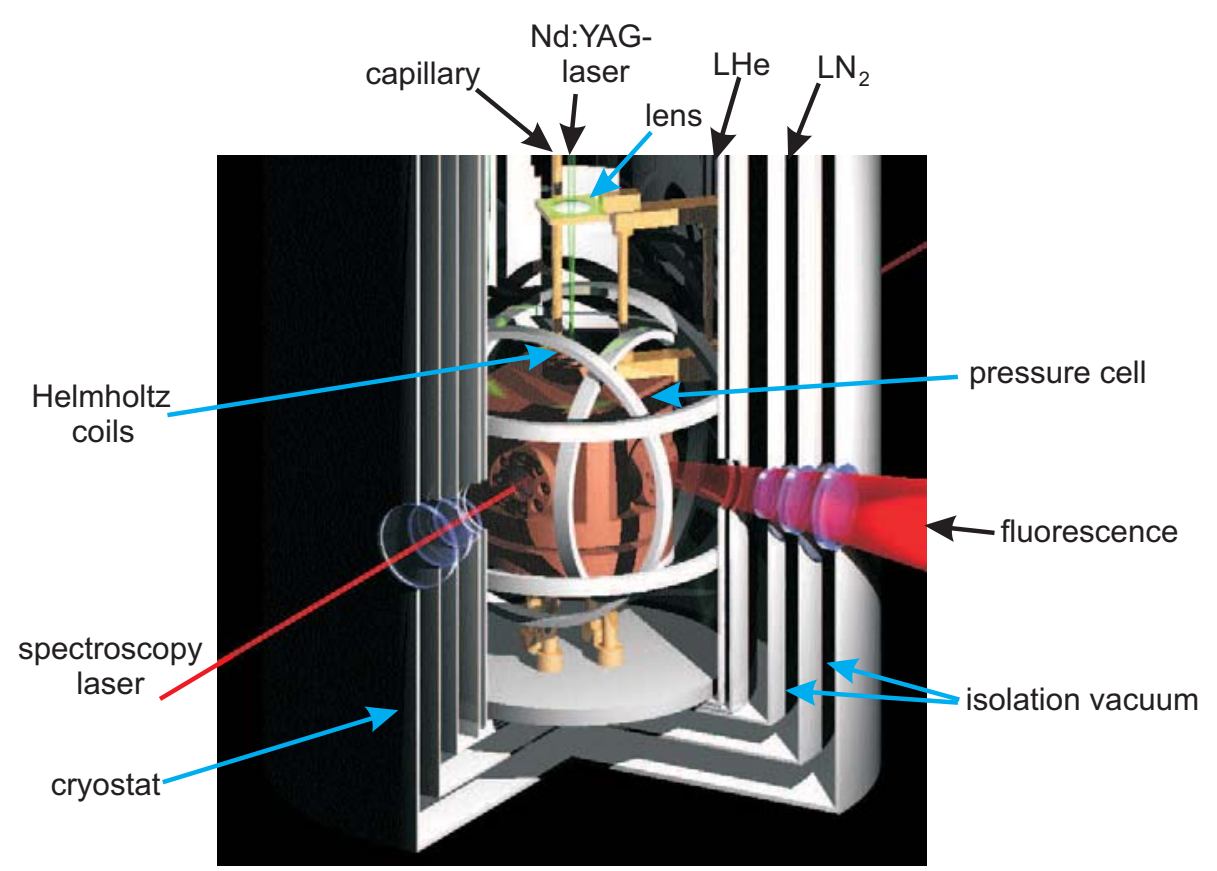

Fig. 12. Vertical cut trough the cryostat, showing the pressure cell and the triaxial system of Helmholz coils inside the helium bath.

stages of the implantation process are shown schematically in the lower panels of Fig. 13. The top row of the figure shows photographs taken through one of the side-windows during the implantation procedure. First, the beam of a frequency doubled Nd:YAG laser $(\lambda=532$ $\mathrm{nm}$, pulse energy $20 \mathrm{~mJ}$, repetition rate $1 \mathrm{~Hz}$ ) is focused by a lens mounted just above the top window of the pressure cell onto the alkali metal target. The heat deposited by each pulse into the sample melts a portion of the crystal just above the target (Fig. 13(a)). Simultaneously with the melting of the crystal, individual atoms, molecules, ions, and clusters are ejected from the target and are distributed throughout the molten helium region by convection (Fig. 13(b)). We displace the focal point of the lens by raising its position above the cell. In this way the molten part of the crystal is moved upwards until the column of liquefied helium reaches the upper end of the window. After reaching the desired column height, the Nd:YAG laser is switched off and the molten He resolidifies, thereby trapping the implanted species in a column-shaped region (Fig. 13(c)). The implanted species are trapped in this region of the matrix and do not escape from the trapping volume for many hours. This represents the main advantage of using solid He as compared to liquid He, where the implanted atoms are quickly washed out of the observation volume by convection.

Depending on implantation conditions (dopant species, He pressure, laser pulse energy, repetition rate and the total number of pulses) the doped part of the crystal has either a greyish or bluish color. This coloring is due to the strong absorption and scattering of light by metallic clusters produced during ablation. The peak of the optical absorption spectrum of this background lies in the near infrared part of the spectrum for Cs and in the red for $\mathrm{Rb}$. The position and shape of the optical extinction spectrum depends on the cluster size distribution. The comparison of measured extinction spectra with calculations based on the Mie theory of light scattering by small particles reveals [127] a typical average cluster size 

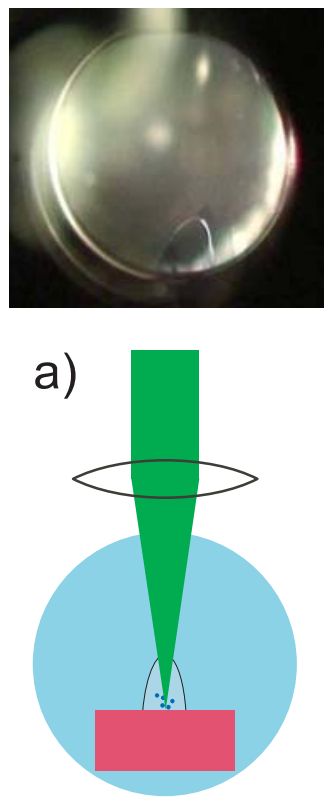

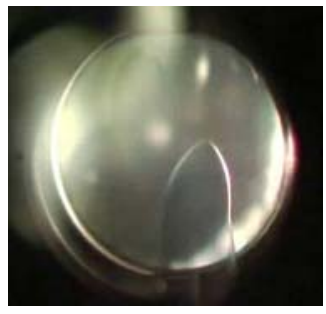

b)

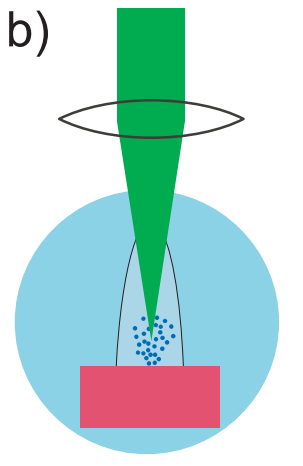

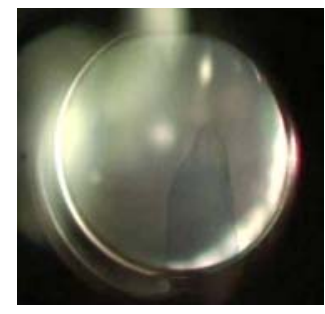

c)

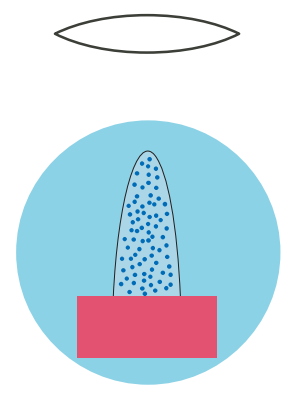

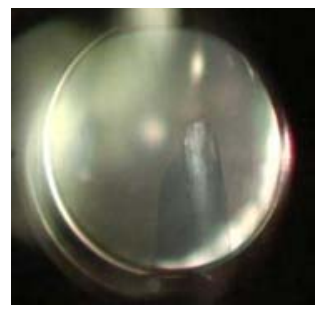

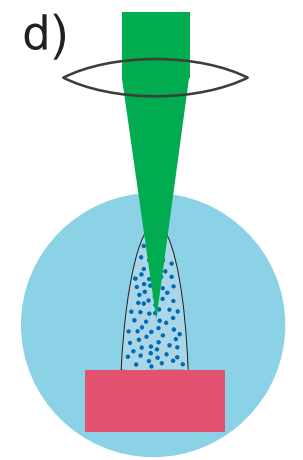

Fig. 13. Different steps of the implantation process shown schematically (lower panels) and as frames of a video recording taken through one of the lateral window of the cryostat and the pressure cell (upper row). In (d) a frame was selected in which fluorescence induced by the Nd:YAG laser can be seen. Details are given in the text.

on the order of $50 \mathrm{~nm}$ and a number density of $\approx 10^{10} \mathrm{~cm}^{-3}$, comparable to or even larger than the density of isolated atoms (typically about $\approx 10^{9} \mathrm{~cm}^{-3}$ ).

The signal of interest for our spectroscopic studies is produced either by atoms or by molecules trapped in the crystal. Because of the softness of the He crystal and because of the large number of defects due to the brute force preparation process, the implanted atoms diffuse within the doped region. When an atom comes near another atom or a cluster, it will be attracted by virtue of the van der Waals force to form a molecule or a larger cluster, thereby decreasing the number of atoms available for the experiments. We found that the number of atoms can be increased again via cluster and molecule dissociation by pulses from the same Nd:YAG laser, focused in the center of the doped region (Fig. 13(d)) with a reduced pulse energy and a lower repetition rate than used for the implantation process (to avoid melting of the crystal).

A different method for introducing impurity particles into superfluid He has been realized in [128], based on the injection of an impurity-helium gas mixture into superfluid He. It was suggested [129] that individual impurity atoms and molecules can be isolated in the helium matrix in this way. However, it was shown later (see Sec. 10.2) that only large impurity clusters are produced and trapped in the matrix in this way. More recently the technique was modified for implanting impurity clusters into solid helium [130,131].

\subsection{Interferometer setup}

In our experiments the refraction index of the sample can be monitored [127] by means of a two-beam interferometer (Mach-Zehnder) shown in Fig. 14, with one arm passing through the sample. The interferometer is illuminated with a green diode laser pointer. The laser beam is expanded to cover the doped and undoped parts of the sample. A small angle is 


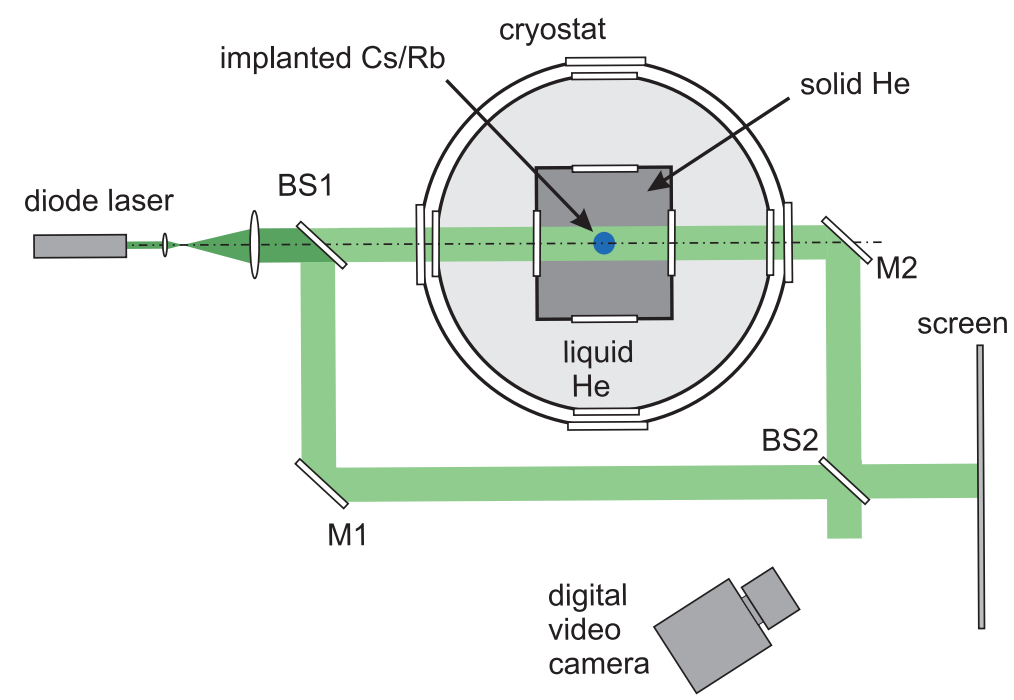

Fig. 14. Horizontal cross section of the cryostat and the pressure cell (not to scale) and top view of the Mach-Zehnder interferometer.
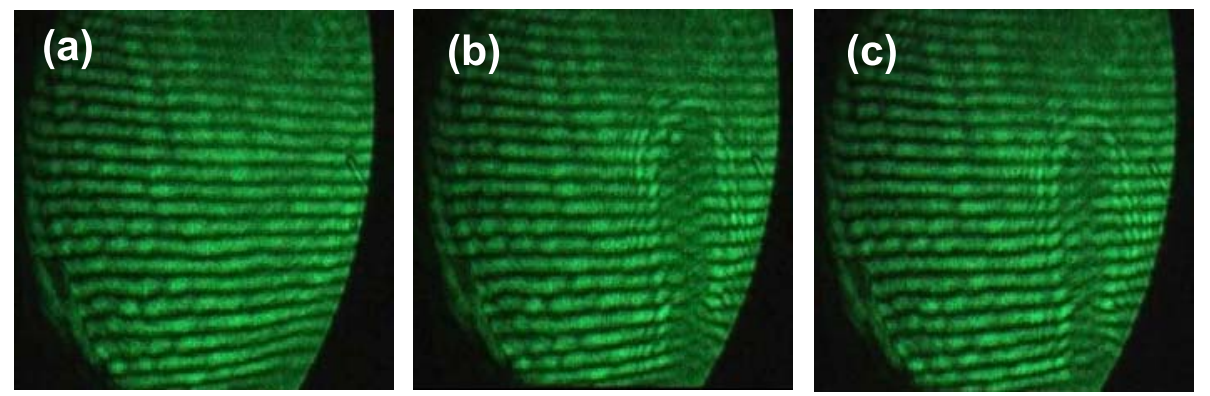

Fig. 15. Fringe pattern obtained with a Mach-Zehnder interferometer during the implantation process. (a) - before the ablation laser pulse; (b) - a few milliseconds after the laser pulse; (c) - about $0.1 \mathrm{~s}$ after the laser pulse.

introduced between the two beams at the combining beamsplitter BS2, in order to obtain a system of horizontal interference fringes with a spacing of about $1 \mathrm{~mm}$. An increase of the helium pressure in the cell results in a uniform shift of the fringe pattern that can be used for very precise calibration of He density changes in the cell. Moreover, any inhomogeneity of the sample results in a distortion of the pattern. As an example we show in Fig. 15 a series of photographs of the fringe pattern taken during the ablation process: before the laser pulse (a), several milliseconds after (b), and about $0.1 \mathrm{~s}$ after the pulse. In Fig. 15(b) and (c) one can clearly see how the He density in the center is perturbed due to the heat deposited by the laser pulse.

\subsection{Setup for optical spectroscopy and time-resolved studies}

A top view of the typical setup for purely optical studies is shown in Fig. 16. The trapped atoms/molecules of interest are excited by a laser beam traversing the doped region of the crystal in a horizontal direction. For cw excitation we use radiation form infrared diode lasers or a tunable $\mathrm{Ti}: \mathrm{Al}_{2} \mathrm{O}_{3}$ laser pumped by a frequency doubled $\mathrm{Nd}: \mathrm{YVO}_{3}$ laser. In recent years we performed most studies with a pulsed optical parametric oscillator (OPO) pumped by the 


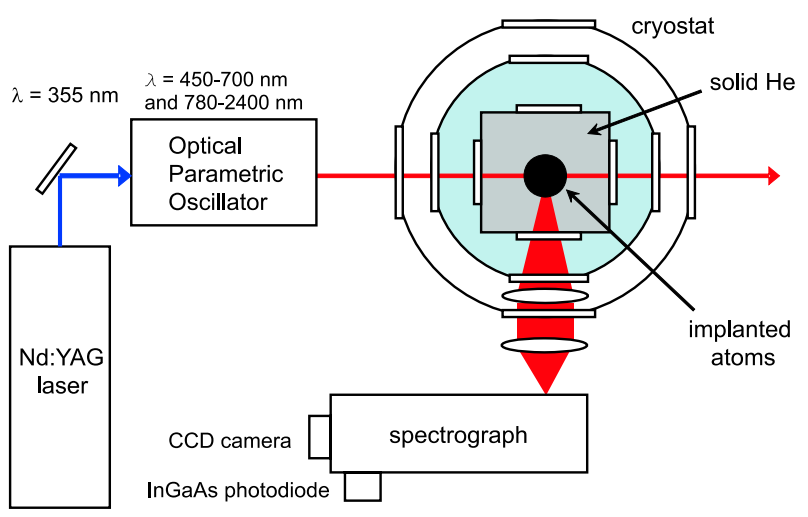

Fig. 16. Horizontal cross section of the cryostat and the pressure cell (not to scale) and top view of the optical spectroscopic setup.

third harmonic $(\lambda=354 \mathrm{~nm})$ of a pulsed Nd:YAG laser. The tuning range of the OPO covers the large spectral intervals of 780-2400 $\mathrm{nm}$ (idler beam) and 450-700 $\mathrm{nm}$ (signal beam). The repetition rate of the laser pulses is $10 \mathrm{~Hz}$ and the average light power delivered to the experiment is controlled by means of a $\lambda / 2$ plate and a polarizer. During the experiments, the OPO power is kept at a level of $1 \mathrm{~mW}$ to prevent melting of the He crystal.

The laser induced fluorescence from the sample volume $\left(\approx 3 \mathrm{~mm}^{3}\right)$ is collimated by a lens mounted inside of the cryostat and is focused into a grating spectrograph with a resolution of $0.2 \mathrm{~nm}$. To access different parts of the spectral domain, two different photodetectors can be mounted on the spectrometer's output port. Visible and near-infrared fluorescence is detected by means of a CCD camera which allows the recording of complete spectra, while for wavelengths above $1 \mu \mathrm{m}$ an InGaAs photodiode is used. In the latter case the grating is rotated by a stepper motor, and the spectra are recorded point by point.

This setup was used in our studies of laser-induced fluorescence of atomic Cs, $\mathrm{Cs}^{*} \mathrm{He}_{N}$ exciplexes [132,68], atomic $\mathrm{Rb}, \mathrm{Rb}^{*} \mathrm{He}_{N}$ exciplexes [113], and $\mathrm{Rb}_{2}$ molecules [133].

For time-resolved measurements two modifications of the same setup were used. The lifetime of the atomic $6 P_{1 / 2}$ state of Cs was studied [134] using a time-resolved single photon counting technique. Radiation from a pulsed diode laser at $850 \mathrm{~nm}$ (10 kHz repetition rate) excited the implanted atoms and a single-photon counting photomultiplier (PM) detected the light analyzed by the spectrograph. The excitation laser is triggered by a pulse generator, a delayed pulse of which is used as a start signal for a time-to-amplitude converter (TAC). The PM pulses produced by the fluorescence light are amplified and discriminated by a constant fraction discriminator (CFD), whose logic output serves as the stop signal for the TAC. A histogram of the amplitudes of the TAC pulses is recorded in 0.4 ns wide time bins using a multiscaling card in a personal computer. This histogram directly reflects the time dependence of the fluorescence intensity. In order to avoid pile-up effects, the light intensity is attenuated such that on average much less than one photon is detected per excitation pulse.

For wavelengths above $900 \mathrm{~nm}$, where the spectral sensitivity of the photomultiplier is too low, another method is used. The implanted species are excited by the much more powerful pulses produced by the OPO. The resulting fluorescence pulses are then recorded by a photodiode mounted at the output slit of the spectrograph and stored in a digital 

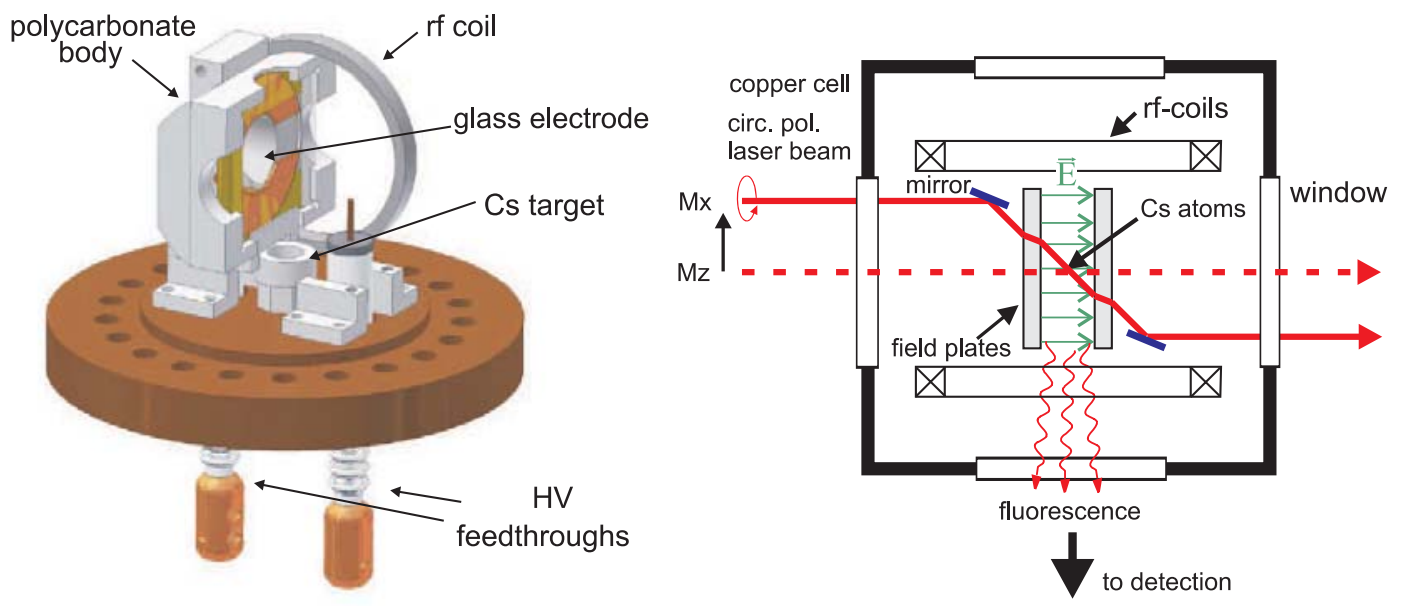

Fig. 17. (left) the bottom flange of the pressure cell of Fig. 12 with one of the rf-coils and one of the HV electrodes shown; (right) top view of the set-up for magnetic resonance experiments with electric fields.

oscilloscope. Lifetimes of $\mathrm{Cs}^{*} \mathrm{He}_{N}$ exciplexes and of excited dimer molecules $\mathrm{Rb}_{2}, \mathrm{RbCs}$ can be measured with this technique.

\subsection{Magnetic resonance spectroscopy}

Optically detected magnetic resonance (ODMR) spectroscopy relies on driving magnetic dipole transitions between Zeeman split levels in combination with optical preparation and detection. This technique which involves a static and an oscillating magnetic field will be discussed in detail in Sec. 9.1. Some of the main elements of the corresponding experimental setup are shown in Fig. 12. The static magnetic field is produced by three pairs of superconducting Helmholtz coils mounted around the pressure cell inside of the He bath. All coil pairs are mutually orthogonal. A highly stable field is produced by operating the coils in a persistent current mode [21]. The recording of ultra-narrow magnetic resonance lines requires special care in the choice of low-temperature (and in some cases high-voltage) compatible non-magnetic materials and in the design of the pressure cell. For example, indium, a most commonly used material for over-/under-pressure seals at low temperatures is superconductive at our experimental conditions. The magnetic flux through the seals becomes frozen during their cooling below the critical temperature, thus producing a strongly inhomogeneous magnetic field that severely perturbs all high-resolution magnetic resonance experiments. In order to avoid this problem, all sealing rings in the pressure cell are made of specially shaped and annealed pure aluminum. Laboratory fields and gradients are suppressed by a cylindrical three-layer magnetic shield surrounding the whole cryostat.

For spin polarizing the sample via optical pumping the latter is irradiated by a circularly polarized cw laser beam resonant with the $D_{1}$ transition of the implanted atoms. For Cs a near infrared diode laser $(1 \mathrm{~mW})$ is used, whereas for $\mathrm{Rb}$ we use $1 \mathrm{~mW}$ of radiation from the Ti:Sa laser. Laser-induced fluorescence from the implanted atoms is collected in the same way as in the optical spectroscopic experiments and detected either by a photomultiplier or by an avalanche photodiode. In these experiments we do not use the spectrograph but rather a narrow-band interference filter to suppress scattered laser light. As discussed in Sec. 7, the 
fluorescence of $\mathrm{Rb}$ atoms in solid $\mathrm{He}$ is so weak that it has escaped observation for a long time. For this reason the first magnetic resonances in $\mathrm{Rb}$ were detected by monitoring the transmitted intensity of the pumping laser.

The magnetic resonance transitions are induced by an oscillating magnetic field produced by a second system of (smaller) Helmholtz coils mounted inside the pressure cell (not shown in Fig. 12). The current to those coils is supplied by a waveform generator through an electric feedthrough at the bottom of the cell. The ODMR technique was also used to study the microwave transition at $9.2 \mathrm{GHz}$ between the two hyperfine levels of the ground state of Cs in solid He. In that case, the oscillating magnetic field was produced by a microwave frequency synthesizer and irradiated through one of the fused silica optical windows of the pressure cell.

A separate subsection of this paper (Sec. 9.7) is devoted to our very recent studies of the Stark effect on the magnetic resonance transitions in Cs. For those studies, the ODMR setup described above was modified so that a large static electric field could be applied to the Cs-doped He crystal. A bipolar high voltage produced by two identical power supplies of opposite polarities was delivered to the sample via HV cables traversing the top flange of the cryostat, then the helium bath, inside of which the high voltage entered the pressure cell via two specially designed (nonmagnetic) feedthroughs mounted on the bottom of the cell (Fig. 17). Inside of the cell the feedthroughs are connected to a pair of electrodes (size $4 \times 4 \mathrm{~cm}$, spacing $6.0 \mathrm{~mm}$ ), cut from floatglass and coated on one side with a transparent conductive tin oxide layer. Pure liquid and solid $\mathrm{He}$ are excellent electric insulators. In practice, the magnitude of the applied electric field strength is limited by the leakage current through the doped He crystal, which melts the crystal and produces an electric breakdown in the cell for field strengths above $50 \mathrm{kV} / \mathrm{cm}$.

\section{Optical spectroscopy}

\subsection{Absorption by electron bubbles}

A first observation of light absorption by electron bubbles in liquid helium was reported in 1967 [135]. In that experiment transitions from the $1 s$ ground state towards the continuum were studied by monitoring changes in the mobility of electrons under illumination by infrared and visible radiation. Spectroscopic studies of the transitions between bound states were reported for liquid $[136,65,137,64]$ and solid $[66,138,139]{ }^{4} \mathrm{He}$. In those experiments the $1 s \rightarrow 1 p$ transition was studied by monitoring either the photocurrent [136], or the transmission of infrared radiation through the sample $[65,137,64,66,138,139]$. The transition wavelength decreases with pressure from $12.4 \mu$ in liquid He at saturated vapor pressure to $3.4 \mu$ in solid He at 100 bar (see Fig. 18), in agreement with theoretical predictions [3638,33 . The lineshape of the transition was obtained either by scanning the wavelength of the light source (a halogen lamp and a monochromator) $[136,65,66,138,139]$ or by changing the helium pressure $[137,64]$. The absorption lineshapes were modeled $[61,62]$ and good agreement with the experimental results of [65] was achieved (see Fig. 18) when breathing and quadrupole oscillation modes of the bubble were taken into account.

The transition $1 s \rightarrow 2 p$ was observed at $2.5 \mu$ in an unpublished study [140], cited in [136]. 


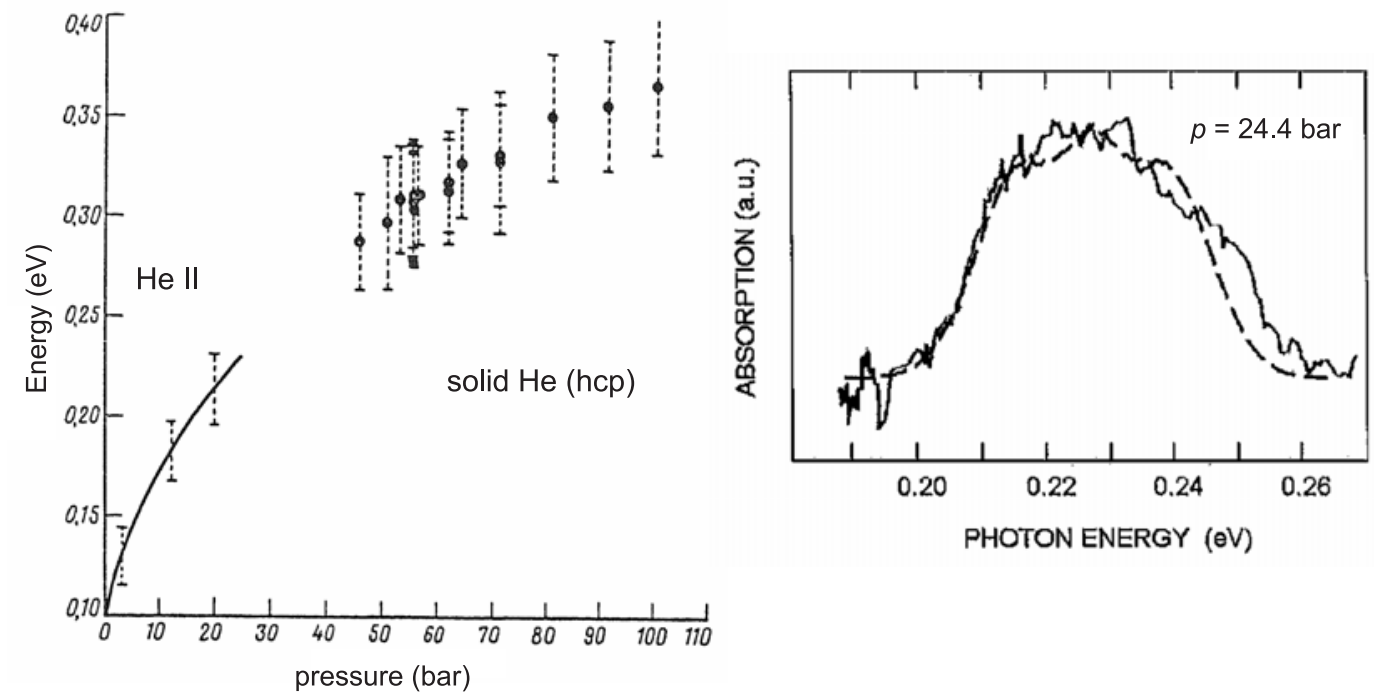

Fig. 18. Left: pressure dependence of the $1 s \rightarrow 1 p$ transition energy of electron bubbles in liquid and solid ${ }^{4}$ He from [66]. Vertical dashed lines represent the resonance linewidths, the solid line is the transition energy in liquid He measured in [65]. Right: The calculated (dashed line) and measured (solid line) lineshape of the $1 s \rightarrow 1 p$ transition in liquid He from [62]. The experimental data are from [65]. Right figure reproduced from [62] with kind permission of Springer Science and Business Media.

The calculation of the transition energies for different pressures of liquid He was performed in $[36-38,33]$.

The effect of light absorption on the $1 s \rightarrow 1 p$ transition in liquid helium was also observed in recent experiments [141-143] using the phenomenon of bubble cavitation at negative helium pressures. During the negative pressure swing (at a helium pressure of -1.85 bar, at $T$ $=1.4 \mathrm{~K})$ in the focus of an acoustic wave, the ground-state electron bubble explodes, a phenomenon that was studied experimentally in $[144,145]$. The excited $1 p$ state is populated by illuminating the sample with powerful $\mathrm{CO}_{2}$ laser radiation, whose wavelength $(\lambda=10.6 \mu \mathrm{m})$ is within the absorption line profile. The excited-state bubble explodes at a smaller negative pressure of about -1.5 bar [143]. By measuring the relative populations of the excited and ground states at different laser powers the authors of [143] have determined the lifetime of the excited state to be $\tau=50 \pm 4$ ns. This experimental lifetime is three orders of magnitude shorter than the radiative lifetime calculated in [77] $(\tau=44 \mu \mathrm{s})$ and [84] $(\tau=60 \mu \mathrm{s})$. The observations thus confirm the prediction of [82] concerning a radiationless decay channel of the excited electron bubble (see Sec. 4.4).

\subsection{Atomic fluorescence in liquid He}

First atomic spectra in liquid He were obtained by bombarding pure liquid He with energetic electrons [146-150]. In those experiments a large number of excited states of the He atom and of $\mathrm{He}_{2}$ excimers were populated and the fluorescence at the $3^{3} S \rightarrow 2^{3} P$, $2^{3} P \rightarrow 2^{3} S, 3^{1} S \rightarrow 2^{1} P$ transitions was observed. A significant population accumulates in the metastable $2^{3} S$ state of He allowing for possible absorption studies of the $2^{3} S \rightarrow 2^{3} P$ transition. In agreement with the predictions [41] of the bubble model both absorption and emission lines shift to shorter wavelengths and are broadened when increasing the helium pressure. 
Table 1

\begin{tabular}{|c|c|}
\hline element & reference \\
\hline $\mathrm{Rb}$ & {$[44-46,94]$} \\
$\mathrm{Cs}$ & {$[44-46]$} \\
$\mathrm{Be}$ & {$[155]$} \\
$\mathrm{Mg}$ & {$[124,125,120,155,53,153,85,54]$} \\
$\mathrm{Ca}$ & {$[124,117,42,55,121,56]$} \\
$\mathrm{Sr}$ & {$[124,117,42]$} \\
$\mathrm{Ba}$ & {$[124,117,42,155,121,119,57,51,1,55]$} \\
$\mathrm{Cu}$ & {$[119,155]$} \\
$\mathrm{Ag}$ & {$[125,155,151,152]$} \\
$\mathrm{Au}$ & {$[119,155]$} \\
$\mathrm{Zn}$ & {$[155]$} \\
$\mathrm{Cd}$ & {$[155]$} \\
$\mathrm{Hg}$ & {$[155]$} \\
$\mathrm{Al}$ & {$[125,155,154]$} \\
$\mathrm{Ga}$ & {$[125]$} \\
$\mathrm{In}$ & {$[125,155]$} \\
$\mathrm{Tl}$ & {$[155]$} \\
$\mathrm{Sn}$ & {$[155]$} \\
$\mathrm{Pb}$ & {$[155]$} \\
$\mathrm{Yb}$ & {$[124,125]$} \\
$\mathrm{Ba}+$ & {$[116]$} \\
$\mathrm{Yb}+$ & {$[58]$} \\
\hline & \\
\hline &
\end{tabular}

Atomic species in liquid He that have been studied by spectroscopic methods.

Spectroscopic studies of impurity atoms in liquid He were reported for a large number of metal atoms. Those experiments were reviewed in $[4,2,5]$. In Table 1 we list the elements studied up to date with the corresponding references. Also included in the table are the experiments by [151], [152] and [153,154] on He nano-droplets doped with $\mathrm{Ag}, \mathrm{Mg}$ and $\mathrm{Al}$ atoms, since those atoms reside inside of the droplet and their observed spectra are very similar to those obtained in bulk liquid helium.

A detailed comparison of experimental and theoretical absorption lineshapes was performed for $\mathrm{Cs}$ and $\mathrm{Rb}[45,46], \mathrm{Ag}[111,110], \mathrm{Yb}^{+}$[58], $\mathrm{Ba}[57,51]$ and $\mathrm{Ca}$ [56]. The splitting of the $n^{2} S_{1 / 2}-n^{2} P_{3 / 2}\left(D_{2}\right)$ transitions in $\mathrm{Cs}, \mathrm{Rb}$, and $\mathrm{Yb}^{+}$by quadrupolar bubble oscillations (see Sec. 4.5) was confirmed experimentally [46,58], while the $n^{2} S_{1 / 2}-\mathrm{n}^{2} P_{1 / 2}\left(D_{1}\right)$ transitions show no splitting. A similar behavior was observed in the earlier work [116] on $\mathrm{Ba}^{+}$ions. The $n^{2} S_{1 / 2}-n^{2} P_{1 / 2,3 / 2}$ transitions of silver in bulk liquid helium [125] and in He nanodroplets [151,152] also show very similar spectral profiles of the absorption lines. The $D_{2}$ transition is split in two partially resolved components separated by approximately 500 $\mathrm{cm}^{-1}$. The structure of He clusters doped with a $\mathrm{Ag}$ atom and the absorption lineshapes were modelled using the quantum Monte Carlo [110] method and path integral molecular dynamics [111] simulations. Both calculations successfully reproduce the main features of the observed lineshapes, but slightly overestimate the blueshifts and the spectral widths of 
the lines compared to those in the free atom.

The effect of bubble deformations on the ${ }^{1} S_{0}-{ }^{1} P_{1}$ transitions of alkali-earth atoms is much less pronounced and results merely in asymmetric lineshapes, as observed for $\mathrm{Mg}$ $[85,153,54]$, Ca $[117,56], \mathrm{Sr}[42]$, and $\mathrm{Ba}[57,51]$. A calculation of lineshapes taking only the breathing mode $[43,57,51]$ of bubble oscillations into account underestimates the asymmetry. A calculation for $\mathrm{Ca}$ [56] that took breathing, dipolar and quadrupolar bubble vibrations into account yielded a better agreement with experimental data. The results of a Monte Carlo simulation of the trapping site of $\mathrm{Mg}$ in a He cluster [156] predict an asymmetric lineshape, in agreement with experimental spectra.

In all systems described above the ground state of the metal atom in the bubble is spherically symmetric. The $3 P$ ground state of $\mathrm{Al}$ atom represents a special case. In the free atom this state is split into $3^{2} P_{1 / 2}$ and $3^{2} P_{3 / 2}$ fine structure components, separated by $112 \mathrm{~cm}^{-1}$. Depending on wether the spin-orbit coupling is destroyed or preserved by the interaction with the surrounding helium, the ground state orbital and the corresponding atomic bubble are either dumbbell-like (spin-orbit uncoupled $P_{z}$ orbital), or spherical $\left({ }^{2} P_{1 / 2}\right.$ orbital). In the former case, the excited $3 D$ state splits into 3 components with $M_{L}=0, \pm 1, \pm 2$ (the fine structure splitting of the $3 D$ state is only $1.3 \mathrm{~cm}^{-1}$ and can be neglected) due to the static bubble deformation. In the latter case, a splitting of the excited state may occur due to bubble oscillations. The experimental absorption spectrum of the $3 P-3 D$ transition [154] reveals an asymmetric lineshape with two partially resolved components, separated by approximately $500 \mathrm{~cm}^{-1}$. The authors of [154] suggest that an uncoupling of the spin-orbit interaction takes place and that a ring of $8 \mathrm{He}$ atoms is formed around the waist of the dumbbell-like $P_{z}$ orbital - a structure similar to that of alkali-He exciplexes discussed below in Sec. 5. However, the results of a recent path integral Monte Carlo simulation [59] show that the spin-orbit coupling plays an important role and that the deformation of the ground state bubble is rather small. With the fine structure of the $3 D$ state taken into account, this state splits into five $M_{J}$ components. As a consequence the absorption line profile calculated in [59] is composed of five overlapping components and has a spectral shift and a total width close to those of the experimental lineshape from [154]. The authors of [59] also reported a calculation of the absorption spectrum of the $3 P-4 S$ transition that strongly underestimates the blueshift observed in experiment [125].

\subsection{Atomic fluorescence in solid $\mathrm{He}$}

First systematic spectroscopic studies of atoms in solid He were carried out in experiments with $\mathrm{Ba}$, for which a good agreement with the predictions of the bubble model was obtained $[67,57,51]$. Later those studies were extended to alkali elements. Here we discuss the absorption and fluorescence of cesium atoms in solid He studied in detail in $[51,68,21,48,157]$. Cesium is the only alkali element that emits a strong atomic fluorescence in a solid He environment. The light alkalis $\mathrm{Li}, \mathrm{Na}$, and $\mathrm{K}$ do not emit any fluorescence neither in solid nor in liquid He. Rb represents an interesting intermediate case: it emits fluorescence in liquid He, but the fluorescence is strongly quenched with increased He pressure [94]. Only recently, a very faint fluorescence of atomic Rb could be observed in solid (hcp) He following excitation of the $D_{1}$ and $D_{2}$ transitions [113]. The quenching of the atomic fluorescence is due to the 

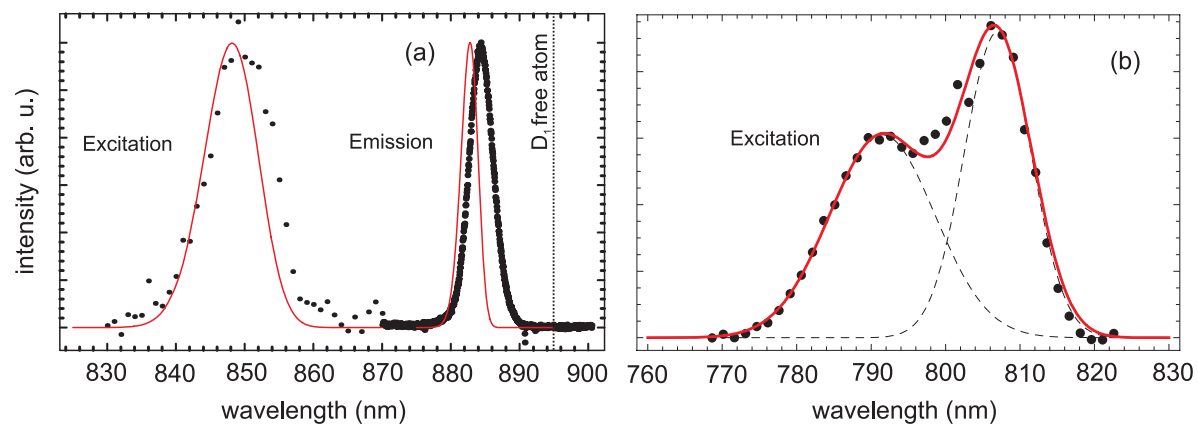

Fig. 19. Atomic absorption and fluorescence spectra of Cs in solid He. (a) Absorption (left) and emission (right) spectra of the $6 S_{1 / 2}-6 P_{1 / 2}\left(D_{1}\right)$ transition in the bcc phase of solid ${ }^{4} \mathrm{He}$ [48]. Dots - experiment, solid lines - theory. (b) the absorption spectrum of the $6 S_{1 / 2}-6 P_{3 / 2}\left(D_{2}\right)$ transition obtained by monitoring the $D_{1}$ fluorescence in hcp phase of solid He [68]. Dots - experiment, solid and dashed lines - fitted Gaussians.

formation of molecular complexes by excited $n P$ states of alkali atoms and the surrounding helium atoms, so-called exciplexes [51,93]. As discussed in Sec. 5 and 7.4, the exciplex formation becomes possible when the alkali-helium interaction exceeds the spin-orbit coupling in the alkali atom. The probability of exciplex formation is thus much larger for lighter alkalis, in which the spin-orbit coupling is weak, and it increases with the density of He, i.e., with rising He pressure, or when going from the liquid to solid phase.

Typical absorption and fluorescence spectra of Cs in solid He are shown in Fig. 19. Only fluorescence on the $D_{1}$ transition can be observed (Fig. 19(a), right), since atoms excited to the $6 P_{3 / 2}$ state are quenched by the interaction with the matrix to form either atoms in the excited $6 P_{1 / 2}$ state or to form $\mathrm{Cs}^{*} \mathrm{He}_{N}$ exciplexes. An absorption spectrum recorded by scanning the frequency of the excitation laser while monitoring the intensity of the $D_{1}$ fluorescence, is shown in the same figure, where both the $D_{1}$ (Fig. 19(a), left) and the $D_{2}$ (Fig. 19(b)) absorption lines are detected. The calculated lineshapes of the $D_{1}$ transition in absorption and emission [48] are also shown in Fig. 19(a) and demonstrate a remarkable agreement with the experimental data. The position of the $D_{1}$ line center measured in absorption and emission is plotted in Fig. 20 as a function of He pressure and density.

As can be seen in Figs. 19 and 20, both the absorption and the emission lines in solid $\mathrm{He}$ are strongly broadened and blueshifted with respect to the free Cs atom. The shift and the broadening are more pronounced in the absorption spectra than in the emission spectra. Very similar spectra were obtained in pressurized liquid He [45], and the corresponding data points are also shown in Fig. 20. The blueshift of the spectral lines can be traced back to changes of the bubble size that occur during the optical absorption-emission cycle, as discussed in detail in Sec. 3.3. The blueshift is due to the fact that the electronic wavefunction of the excited $6 P$ state of Cs spreads over a larger volume than that of the ground state. The overlap of the atomic wavefunction with the He bulk shifts both levels towards higher energies, an effect which is more pronounced for the excited state than for the ground state, because of its larger overlap with the surrounding helium: As a consequence the transition wavelength shifts to the blue. The absorption takes place in a smaller bubble, whose equilibrium size is determined by the Cs ground state and the blue shift is particularly large. The emission occurs in a larger bubble that reflects the size of the excited state and the interaction-induced broadening and shift of the emission lines are smaller. An additional lineshift arises due to a so-called cavity effect, i.e. the interaction of the excited atom with its own radiation reflected at the bubble 

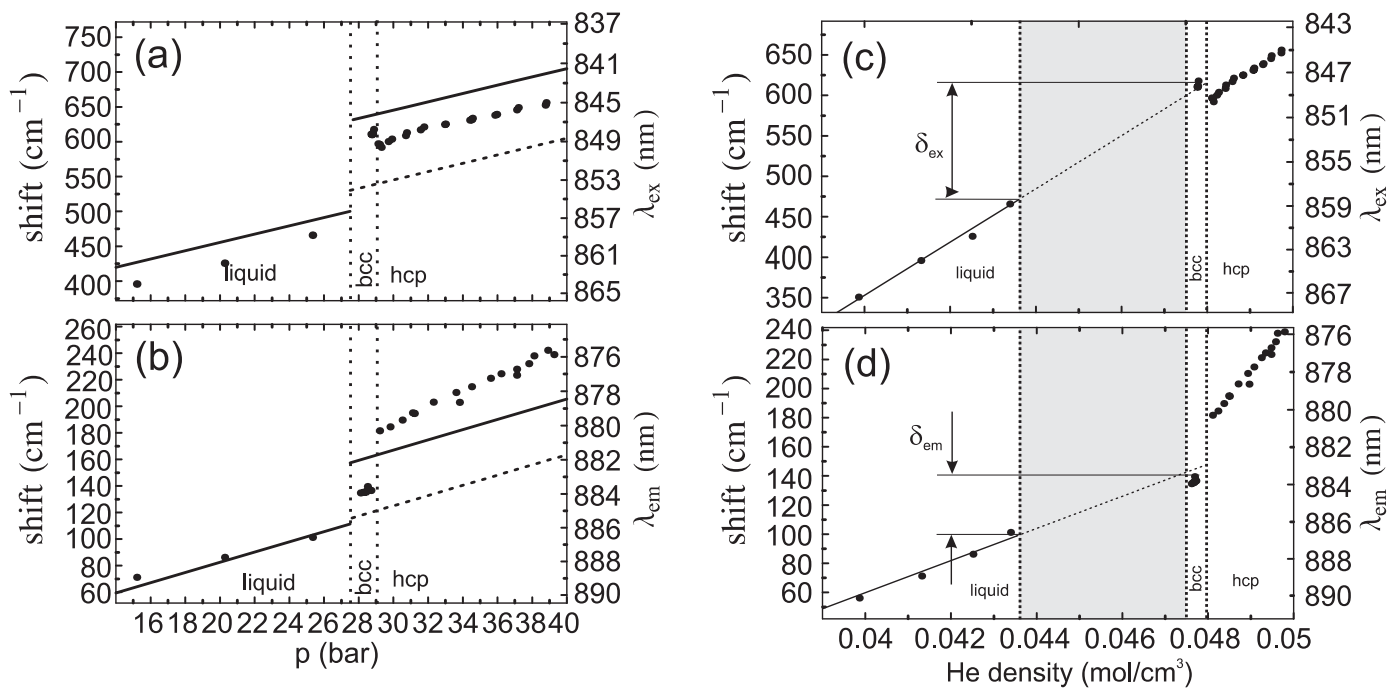

Fig. 20. Dependence of the $D_{1}$ absorption (a), (c) and emission (b), (d) lines of Cs in solid He on He pressure (a), (b) and on He density (c), (d). The experimental data are shown as dots, the solid lines are resonance positions calculated [48] using the spherical bubble model, and the dashed lines show the calculation without taking into account the elastic deformation of the solid. The symbols $\delta_{e x}$ and $\delta_{e m}$ denote the shifts of the resonance energies/wavelengths due to the discontinuity of the He density at the phase boundary. The data points in the liquid phase are taken from [45]. The $D_{1}$ transition in the free Cs atom is at $894 \mathrm{~nm}\left(11186 \mathrm{~cm}^{-1}\right)$.

interface. The effect is rather small: $44 \mathrm{~cm}^{-1}$ in emission and $83 \mathrm{~cm}^{-1}$ in absorption and has an opposite sign (redshift) partially compensates the main effect discussed above.

The bubble model also explains the observed increase of the spectral width and the rate at which the lines shift with He pressure, as shown in Fig. 20(a, b). The abrupt changes of the absorption/emission wavelengths at the liquid-bcc phase transition result from a sudden change of the bubble size at the solidification point discussed in Sec. 3.4. When the same data are plotted as a function of He density (Fig. 20(c, d)), the points obtained in liquid and bcc solid He lie on the same straight line. Calculations [48] assuming isotropic elastic properties of the matrix demonstrate a very good agreement with the experimental results obtained in liquid and in bcc solid He. As discussed in Sec. 4.3, the anisotropy of the elastic properties of the bcc matrix perturbs the $6 P_{1 / 2}$ and $6 S_{1 / 2}$ states only in second order and does not produce any significant effect on the scale of Fig. 20.

The situation is different when one considers the absorption and emission in the hep phase. As discussed in Sec. 4.3.2, the $6 P_{3 / 2}$ state shows already a first order perturbation that results in a splitting of the $D_{2}$ absorption line, that is nicely visible in Fig. 19 (b). The second order perturbation of the $6 P_{1 / 2}$ state is particularly large due to the small energy difference between this state and the $6 P_{3 / 2}$ state. It results in an abrupt shift of the absorption and emission wavelengths of the $D_{1}$ transition at the bcc-hcp phase boundary (Fig. 20). As one can see in Fig. 20(c, d), these shifts are not related to the change of He density at the phase transition.

The only atoms from which optical absorption and emission spectra in solid helium were studied are ${ }^{85} \mathrm{Rb},{ }^{87} \mathrm{Rb},{ }^{133} \mathrm{Cs},{ }^{137} \mathrm{Ba}$, and ${ }^{169} \mathrm{Tm}$. While the (optical) valence electron of alkali atoms interacts with the helium matrix with an energy comparable to the spin-orbit interaction, the optical electron of the lanthanide atom ${ }^{169} \mathrm{Tm}$ behaves quite differently. Its optical transition occurs between two unfilled shells (4f and $5 \mathrm{~d}$ ) which are shielded from the matrix by outer electrons which do not participate in the optical transition. Ishikawa et al. 
[158] have measured absorption and emission lines from Tm in superfluid and solid helium as well as excited state lifetimes. Because of the shielding from matrix effects the authors of [158] could observe widths of optical resonance lines on the order of $0.1 \mathrm{~nm}$ (limited by the resolution of their spectrometer). To our knowledge there exist only two experiments $[158,157]$ besides our own which have reported an optical study of atoms in solid helium.

\subsection{Alkali-helium exciplexes}

The first experimental observation of alkali-helium exciplexes in liquid He was obtained by a group in Kyoto in a series of experiments that covered all alkali elements in liquid He and cold He gas $[99,104,105]$. Such complexes were also observed and extensively studied in experiments with alkali doped superfluid helium nano-droplets [95-98,159-161]. We also mention the related experimental studies of $\mathrm{Ag}^{*} \mathrm{He}_{2}$ [100] and $\mathrm{Mg}^{*} \mathrm{He}_{N}$ [85,54] exciplexes in liquid $\mathrm{He}$ and a study of $\mathrm{Ba}^{+*} \mathrm{He}[102,103]$ in cold He gas. In solid helium, the formation of $\mathrm{Cs}^{*} \mathrm{He}_{N}$ exciplexes was reported by our group for the first time in [132] and described with more details in $[112,68]$. More recently we have extended those studies to $\mathrm{Rb}^{*} \mathrm{He}_{N}$ in solid He [113].

The exciplexes are typically detected via their laser-induced fluorescence which can be excited at the wavelengths of the resonant atomic transitions. The characteristic feature of exciplex emission, and the reason why it has escaped observation for many years, is its strong red shift with respect to the corresponding absorption lines. For instance $\mathrm{Cs}^{*} \mathrm{He}_{N}$ can be formed after excitation at $800 \mathrm{~nm}$, while it fluoresces at $1400 \mathrm{~nm}$. Typical spectra of $\mathrm{Cs}^{*} \mathrm{He}_{N}$ and $\mathrm{Rb}^{*} \mathrm{He}_{N}$ in the hcp phase of solid He excited at the $D_{2}$ atomic transitions of Cs and Rb, respectively, are shown in Figs. 21(a) and 21(b). In agreement with the discussion of Sec. 5, we have identified the diatomic and triatomic exciplexes $\operatorname{Cs}\left(6 P_{3 / 2}\right) \mathrm{He}_{2}, \mathrm{Rb}\left(5 P_{3 / 2}\right) \mathrm{He}_{1}$, and $\mathrm{Rb}\left(5 P_{3 / 2}\right) \mathrm{He}_{2}$ via their emission lines at 952,806 , and $847 \mathrm{~nm}$, respectively (Fig. 21). The two strongest and most redshifted emission bands originate from ring-shaped complexes. In liquid and gaseous He such exciplexes were observed for all alkali elements excluding Cs, for which only linear diatomic and triatomic complexes were found.

For $\mathrm{Rb}$ in liquid $\mathrm{He}$ at saturated vapor pressure [104], and for $\mathrm{Rb}$ on He nano-droplets [98] exciplex formation was observed only following excitation of the $\mathrm{Rb}$ atom to the $5 P_{3 / 2}$ state ( $D_{2}$ excitation). When the $5 P_{1 / 2}$ state is excited ( $D_{1}$ excitation), exciplex formation is possible only via a tunneling transition through the potential barrier in the $A \Pi_{1 / 2}$ state (analogous to that shown in Fig. 10). As calculated in [97], the exciplex formation time in that case is much larger than the radiative lifetime of the excited $\mathrm{Rb}$ atom, thus preventing exciplex formation. However, in gaseous He, there is a sufficient number of energetic He atoms that are able to pass the barrier, so that exciplexes can be formed (and observed) in that environment [104]. In solid $\mathrm{He}$, on the other hand, $\mathrm{Rb}^{*} \mathrm{He}_{6}$ exciplexes have been observed [113] after laser excitation of either the $6 P_{3 / 2}$ or the $6 P_{1 / 2}$ state. According to the bubble model, the closest neighboring He atom is separated from the $\mathrm{Rb}$ atom by a distance given by the bubble radius $R_{b}$, which is smaller in solid He than in liquid He. Our calculations show that for $\mathrm{Rb}$ the potential barrier in the $A \Pi_{1 / 2}$ state is located outside the bubble interface, so that no tunneling is involved in the exciplex formation. Any static or dynamic bubble deformation with an axial symmetry should result in a complete spin-orbit decoupling and 


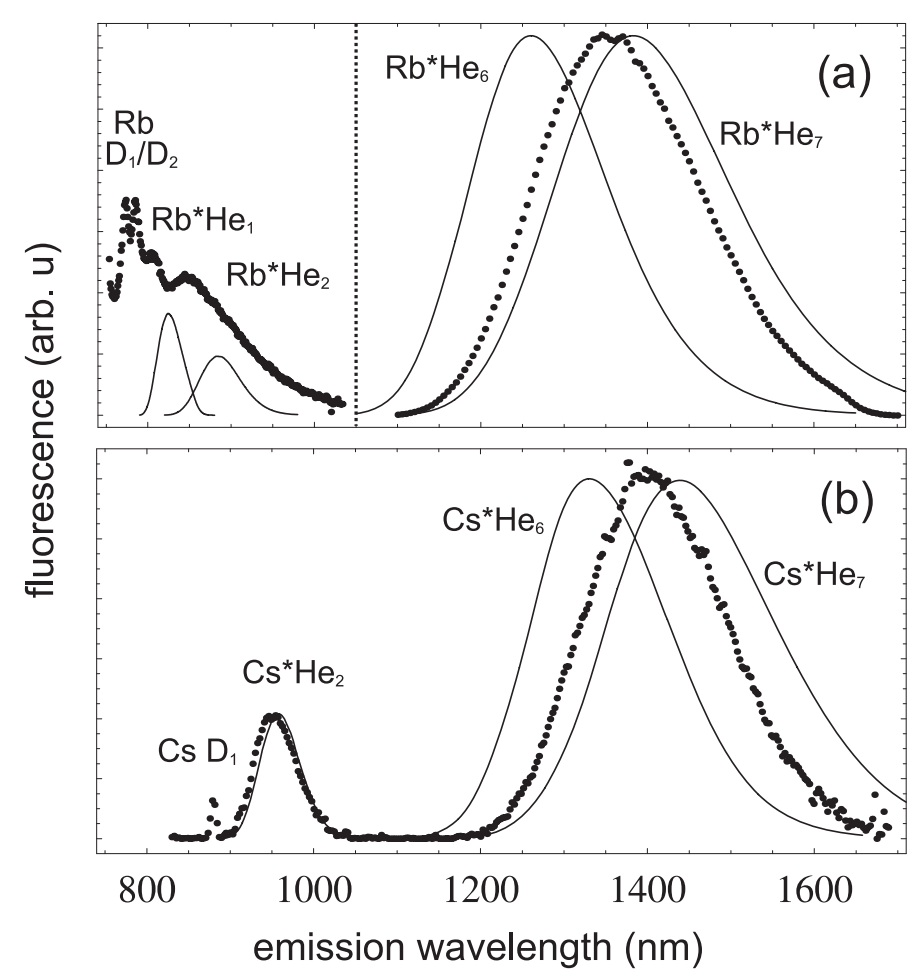

Fig. 21. Experimental emission spectra of $\mathrm{Rb}^{*} \mathrm{He}_{N}$ (a) and $\mathrm{Cs}^{*} \mathrm{He}_{N}$ (b) exciplexes in hcp solid He excited at the $D_{2}$ transitions of $\mathrm{Rb}$ and $\mathrm{Cs}$, respectively. The atomic $D_{1}$ emission line of Cs and the $D_{1} / D_{2}$ doublet of Rb are visible on the blue side of the exciplex spectra. The vertical dotted line in (a) separates two spectral regions with different vertical scales. The emission of atomic $\mathrm{Rb}$ and $\mathrm{Rb}^{*} \mathrm{He}_{1,2}$ exciplexes on the left part of the figure is several orders of magnitude weaker than any other spectral feature.

the formation of a ring of He atoms around the waist of the perturbed $5 P_{1 / 2}$ orbital. This process is so efficient that no intermediate products $\mathrm{Rb}^{*} \mathrm{He}_{n}(n<6)$ are observed [113], except for a very faint atomic fluorescence at the $D_{1}$ line. The onset of this new exciplex formation channel occurs most likely in liquid He, when the He pressure is increased above 15 bar, as evidenced by the corresponding quenching of the atomic fluorescence in that pressure range [94].

For Cs atoms the potential barrier in the $A \Pi_{1 / 2}$ state (see Fig. 10) is higher than for $\mathrm{Rb}$ and the corresponding bubble radius is larger. A tunneling transition is required for exciplex formation via $D_{1}$ excitation and the subsequent fluorescence is very weak [68] even in solid He. On the other hand, the formation channel via $D_{2}$ excitation followed by a fine-structure relaxation is very efficient. It is interesting to note that the static quadrupolar deformation of the bubble in the hcp phase induces a partial decoupling of the spin-orbit interaction even in the absence of bubble shape oscillations which results in an enhanced efficiency for the exciplex formation. The effect manifests itself in the atomic/exciplex fluorescence ratios in bcc and hcp phases.

Owing to the very high He density and the compact bubble structure in solid helium, solid helium matrix isolation spectroscopy has proven - in comparison to other experimental techniques, such as doped helium nano-droplets and cold helium gas - to be particularly well suited for the formation and investigation of exciplexes with a maximally allowed number of bound He atoms. Due to the very high collision rate with surrounding helium atoms, 
the attachment of He atoms in solid He proceeds at a much faster rate than in any other environment and stops only when all vacancies are occupied, i. e., when the two dimples of the $n P_{3 / 2}$ orbital or of the ring-shaped belt around the waist of the perturbed $n P_{1 / 2}$ orbital are filled. All intermediate complexes occur only as short-lived transients which have no time to fluoresce and which thus do not contribute to the emission spectrum. A similar behavior was observed in liquid He $[99,104,105]$, with the difference that in this case the perturbation of the $6 P_{1 / 2}$ state of $\mathrm{Cs}$ is not strong enough to allow the formation of the ringshaped complex. The largest complex seen in superfluid helium is the diatomic $\mathrm{Cs}\left(6 P_{3 / 2}\right) \mathrm{He}_{2}$ exciplex [99].

\section{5. $H e_{2}^{*}$ excimers}

Fluorescence of $\mathrm{He}_{2}^{*}$ excimers had been observed already in the first spectroscopic studies of liquid He (for a review see [150,162]). In those experiments [89,163-165] liquid He was bombarded by energetic electrons which excited a number of bound states of the $\mathrm{He}_{2}^{*}$ quasimolecule. The bound-free transition between the lowest singlet excited state $A^{1} \Sigma_{u}$ and the repulsive ground $X^{1} \Sigma_{g}$ state lies in the VUV range at $80 \mathrm{~nm}$ [163-165]. Besides that a number of transitions between excited states were observed [89]. More recently, He 2 fluorescence was observed in liquid He excited by nonresonant intense ultrashort laser pulses [166] and in He droplets excited by synchrotron radiation [167].

The lowest triplet state of $\mathrm{He}_{2}, a^{3} \Sigma_{u}$ is metastable and has a lifetime of $0.1 \mathrm{~s}$ in liquid He [168]. During the electronic bombardment it accumulates a significant population. Absorption spectra of the $a^{3} \Sigma_{u} \rightarrow b^{3} \Pi_{g}$ and $a^{3} \Sigma_{u} \rightarrow c^{3} \Sigma_{g}$ transitions were studied in $[146,168,147,149,169-171]$. Transitions with absorption from the $a^{3} \Sigma_{u}$ state were used in [172] to monitor the dynamics of the He bubble at picosecond time scales (see Sec. 11.4).

All observed emission bands are blueshifted by several $\mathrm{cm}^{-1}$ and have a spectral width of approximately $2 \mathrm{~nm}$ and the vibrational structure of most triplet and singlet bands could be resolved. In addition, the rotational structure of the infrared $a^{3} \Sigma_{u} \rightarrow b^{3} \Pi_{g}$ band was resolved in absorption studies. The shifts and linewidths of the absorption spectra are larger than of the fluorescence spectra. Under increasing He pressure [149] all fluorescence lines shift towards shorter wavelengths at the same rate of $3.2 \AA$ /bar. In absorption the pressure dependence is different: the $a^{3} \Sigma_{u} \rightarrow c^{3} \Sigma_{g}$ band shifts at a rate of $4 \AA /$ bar, while the $a^{3} \Sigma_{u} \rightarrow$ $b^{3} \Pi_{g}$ does not shift at all. The same behavior of the absorption lines was observed in solid He [173]. The wavelengths of the $a^{3} \Sigma_{u} \rightarrow c^{3} \Sigma_{g}$ transition in absorption and of the $d^{3} \Sigma_{u} \rightarrow b^{3} \Pi_{g}$ emission band show a linear dependence on the helium density without any discontinuity at the liquid-solid phase transition.

\subsection{Hydrogen and alkali dimers}

Fluorescence spectra of the $e^{3} \Sigma_{u} \rightarrow a^{3} \Sigma_{g}$ band of the hydrogen molecule in liquid helium were obtained in [90]. The experiment was carried out in normal fluid helium at $4 \mathrm{~K}$. Both vibrational and rotational structures of the band could be resolved. The lines are blueshifted by a few $\mathrm{cm}^{-1}$ and have a spectral width of about $30 \mathrm{~cm}^{-1}$.

Absorption and emission spectra of molecules (dimers) were extensively studied in experiments with helium nano-droplets [174-178,161,179], where all homonuclear and some 


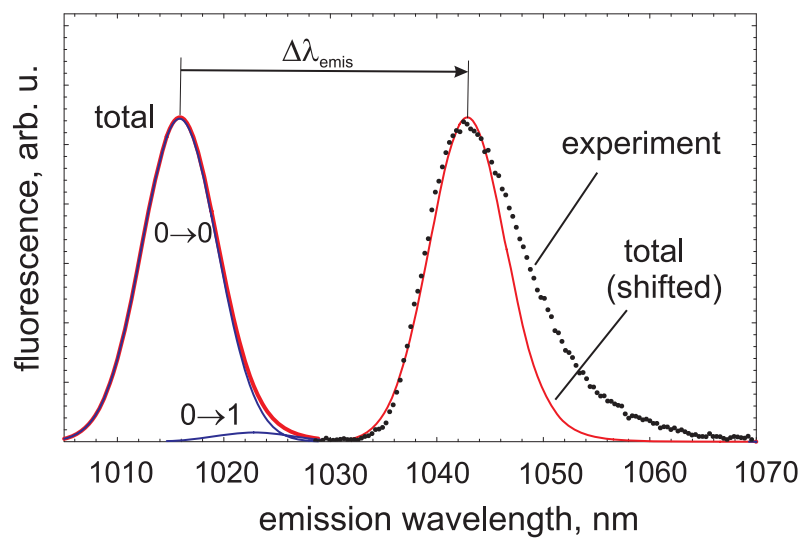

Fig. 22. Experimental (points) and calculated (solid curves) emission spectra of the $(1)^{3} \Pi_{u} \rightarrow X^{1} \Sigma_{g}$ transition in Rb $b_{2}$ The theoretical spectrum (red curve) was obtained as the sum of two vibronic components (blue curves).

heteronuclear alkali dimers were formed and investigated. However, until recently, there have only been very few investigations of metal dimers in bulk condensed helium. In superfluid He only the $X^{1} \Sigma_{g}-A^{1} \Sigma_{u}$ band of $\mathrm{Ca}_{2}$, the $X^{1} \Sigma_{g}-B^{1} \Sigma_{u}$ band of $\mathrm{Cu}_{2}$ [180], and some (unassigned) bands of $\mathrm{Na}_{2}$ and $\mathrm{Li}_{2}$ [122] have been reported.

In our recent experiments [133] we observed for the first time alkali molecules in solid He. In those experiments we studied the fluorescence spectrum of Rb-doped solid He under laserexcitation in the broad spectral range from 450 to $1000 \mathrm{~nm}$ and found a quite remarkable result. Besides atomic and exciplex emission, a single additional spectral feature was observed at $1042 \mathrm{~nm}$ (Fig. 22). We have assigned this emission band to the forbidden $X^{1} \Sigma_{g} \leftarrow(1)^{3} \Pi_{u}$ transition of $\mathrm{Rb}_{2}$. We base this assignment on the spectral structure of the free dimer and on the observation of a long lifetime of the fluorescing state which points to its metastable character (see Sec. 8.3). The calculated spectral profile shown in Fig. 22 is obtained as the sum of all transitions from the lowest vibration state, the only one that is populated at the temperature of the experiment. Each component is represented by a Gaussian spectral profile with a width of $7 \mathrm{~nm}$, typical for atomic fluorescence lines in solid He (see Sec. 7.3), and weighted with the corresponding Franck-Condon factor. As one can see in the figure, only the $0 \rightarrow 0$ and $0 \rightarrow 1$ components contribute significantly to the spectrum, which makes the band rather narrow and a little asymmetric. The spectral shift $\Delta \lambda=20 \mathrm{~nm}$ is attributed to the interaction with the matrix.

Our most striking observation is the fact that this (single) emission band can be excited on 9 distinct absorption bands of $\mathrm{Rb}_{2}$ in the range of 450 to $900 \mathrm{~nm}$ (Fig. 23), including transitions originating from the $X^{1} \Sigma_{g}$ ground state and from the lowest triplet state $(1)^{3} \Sigma_{u}$. The measured and calculated spectral positions of these bands are given in Table 2 . The calculation of the theoretical band positions assumes that only the lowest vibrational state of the initial electronic level is populated, but does not take the interaction with the He matrix into account. We attribute the (small) discrepancies between the calculated and measured band positions to this fact.

Very similar results were also obtained for the heteronucleous dimer RbCs. In that study the sample was doped with a 50:50 mixture of $\mathrm{Rb}$ and $\mathrm{Cs}$. The observed fluorescence is centered at $1160 \mathrm{~nm}$, redshifted by $18 \mathrm{~nm}$ with respect to the forbidden $X^{1} \Sigma \leftarrow(1)^{3} \Pi$ transition in the free molecule. The spectral shift, the lineshape and the decay time of this 


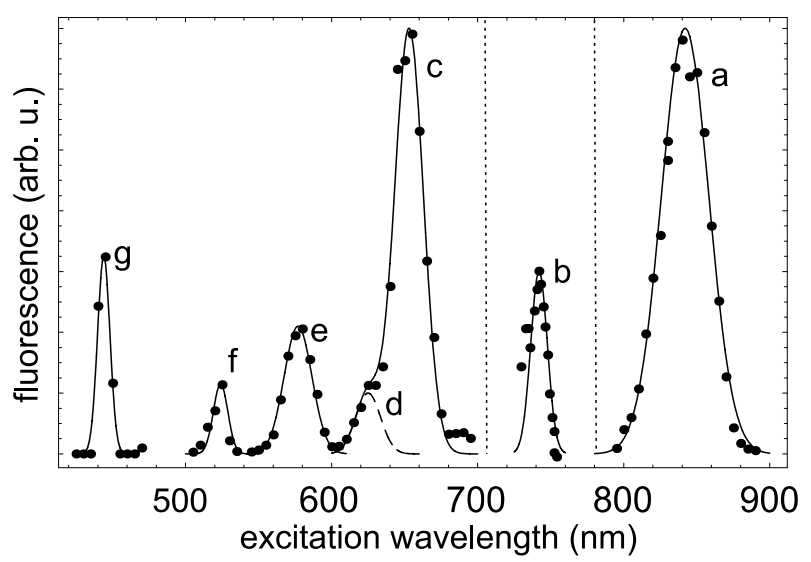

Fig. 23. Measured excitation spectrum of the $\mathrm{Rb}_{2}$ fluorescence at $1042 \mathrm{~nm}$. Experimental data are shown as points, and the solid lines are fitted Gaussians. The assignment of all peaks is given in Table 2. The dotted vertical lines mark the spectral regions covered by the three excitation sources described in the text. The vertical scales differ for the different intervals. Note that the excitation at the bands "b", "d", and "e" also branches to photodissociation channels.

\begin{tabular}{|c|c|c|c|}
\hline band & label & $\lambda_{\text {theor }}$ & $\lambda_{\text {exper }}$ \\
\hline \hline$X^{1} \Sigma_{g} \rightarrow A^{1} \Sigma_{u}$ & $\mathrm{a}$ & 878 & 842 \\
\hline$(1)^{3} \Sigma_{u} \rightarrow(1)^{3} \Pi_{g}$ & $\mathrm{~b}$ & 735 & 742 \\
\hline$X^{1} \Sigma_{g} \rightarrow B^{1} \Pi_{u}$ & $\mathrm{c}$ & 664 & 653 \\
\hline$(1)^{3} \Sigma_{u} \rightarrow(3)^{1} \Sigma_{g}$ & $\mathrm{~d}$ & 623 & 622 \\
\hline$(1)^{3} \Sigma_{u} \rightarrow(2)^{3} \Sigma_{g}$ & $\mathrm{e}$ & 590 & 580 \\
\hline$(1)^{3} \Sigma_{u} \rightarrow(2)^{3} \Pi_{g}$ & $\mathrm{e}$ & 586 & 580 \\
\hline$(1)^{3} \Sigma_{u} \rightarrow(3)^{3} \Sigma_{g}$ & $\mathrm{f}$ & 507 & 524 \\
\hline$X^{1} \Sigma_{g} \rightarrow(2)^{1} \Sigma_{u}$ & $\mathrm{~g}$ & 477 & 445 \\
\hline$X^{1} \Sigma_{g} \rightarrow(2)^{1} \Pi_{u}$ & $\mathrm{~g}$ & 464 & 445 \\
\hline
\end{tabular}

Table 2

Calculated and measured wavelengths of $\mathrm{Rb}_{2}$ absorption bands (in $\mathrm{nm}$ ) in solid He. The labels refer to the corresponding peaks in Fig. 23.

fluorescence band are similar to those of the $X^{1} \Sigma_{g} \leftarrow(1)^{3} \Pi_{u} \mathrm{Rb}_{2}$ transition. Its excitation spectrum contains 5 bands, which can be assigned to known allowed transitions of RbCs. Finally, very recently one more spectral feature was observed in a Cs-doped sample, that can be assigned to the $X^{1} \Sigma_{g} \leftarrow(1)^{3} \Pi_{u}$ transition of $\mathrm{Cs}_{2}$. This fluorescence is much weaker than the corresponding bands of $\mathrm{Rb}_{2}$ and RbCs. Moreover, its spectrum, centered at 1272 $\mathrm{nm}$, overlaps with the strong broadband emission of the $\mathrm{Cs}^{*} \mathrm{He}_{N}$ exciplex, that makes the quantitative study very difficult. The preliminary results show that its spectral width and the lifetime have the same order of magnitude as those of $\mathrm{Rb}_{2}$ and $\mathrm{RbCs}$.

All these experimental observations show that in solid He, due to the interaction with the matrix, all laser-excited molecular states are quenched. The quenching populates of the metastable $(1)^{3} \Pi_{u}$ state which is the lowest excited state in all alkali dimer molecules. The perturbation of the molecule by the surrounding helium partly lifts the selection rule that forbids the radiative transition from the triplet state to the singlet ground state so that the transition $X^{1} \Sigma_{g} \leftarrow(1)^{3} \Pi_{u}$ can be observed.

We have also observed photodissociation of the $\mathrm{Rb}_{2}$ molecule into two $\mathrm{Rb}$ atoms, one in the 


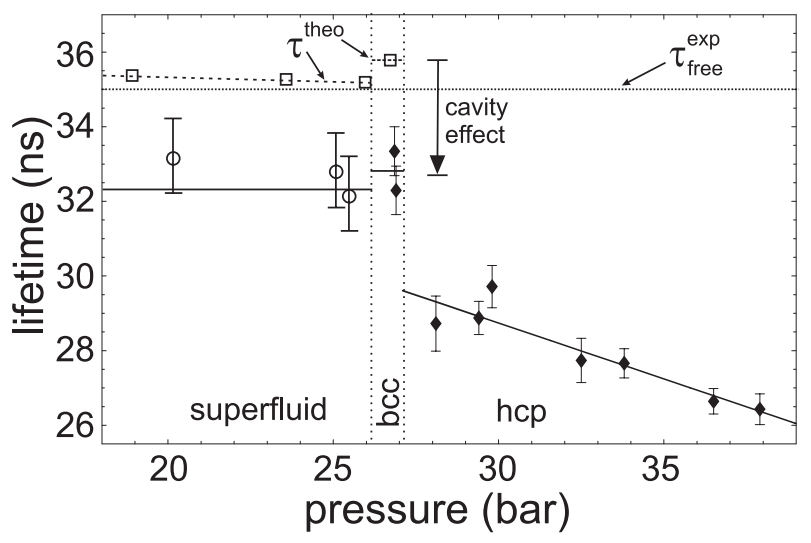

Fig. 24. Pressure dependence of the Cs $6 P_{1 / 2}$ lifetime in solid and liquid He. The open circles are experimental values from [94] and the horizontal dashed line through these points represents their average value including points down to zero pressure not shown in the graph. The vertical dotted lines mark different phase boundaries of condensed He. The horizontal dotted line at $34.8 \mathrm{~ns}$ indicates the lifetime of the free Cs atom. The open squares are theoretical lifetimes.

ground state and the other in the excited $5 P_{1 / 2}$ or $5 P_{3 / 2}$ state. The latter emits fluorescence at the same wavelength as observed from individual $\mathrm{Rb}$ atoms excited either at the $D_{1}$, or the $D_{2}$ transition. The photodissociation spectrum recorded by scanning the excitation wavelength and detecting the atomic fluorescence contains the forbidden molecular bands $(1)^{3} \Sigma_{u} \rightarrow(2)^{3} \Sigma_{u}(650 \mathrm{~nm})$ and $X^{1} \Sigma_{g} \rightarrow(3)^{1} \Sigma_{g}(490 \mathrm{~nm})$ in addition to the bands "b", "d", and "e" shown in Fig. 23 and Table 2.

\section{Time-resolved studies}

\subsection{Lifetime of Cs $6 P_{1 / 2}$ state}

Time-resolved fluorescence of atoms embedded in liquid He was reported in $[125,120,94]$ for bulk liquid and in $[153,154]$ for He nano-droplets. The Cs $6 P_{1 / 2}$ lifetime was found [94] to be pressure independent up to the solidification point, with a value slightly below the free atomic lifetime, while the lifetime of the corresponding $5 P_{1 / 2}$ state of $\mathrm{Rb}$ decreasd with increasing pressure, leading to a complete quenching of the fluorescence. The authors of [94] interpreted their observations as being due to the formation of exciplexes, a deexcitation channel which is open only for $\mathrm{Rb}$ since the corresponding potential barrier for exciplex formation in $\mathrm{Rb}$ is lower than in $\mathrm{Cs}$ (see discussion in Sec. 7.4).

An experimental study of the Cs $6 P_{1 / 2}$ lifetime in solid He has been presented in [134]. In that study a time-correlated photon counting technique was used and the data were taken both in bcc and hcp solid He matrices. The resulting pressure-dependence of the lifetime $\tau$ is shown in Fig. 24 together with the data obtained in [94] in liquid He. The value of $\tau$ in bcc coincides with that in liquid He. It suddenly decreases at the phase transition to the hcp crystal and further decreases when increasing the He pressure.

The results of a theoretical calculation based on the spherical bubble model developed in [48] (described in Sec. 3.4) are also shown in Fig. 24. The constant value of the lifetime over the broad range of He pressures is a consequence of the cancelation of two effects. On one hand, the transition dipole moment $d=\left|\left\langle 6 S_{1 / 2}|e r| 6 P_{1 / 2}\right\rangle\right|$ calculated for the electronic wavefunctions perturbed by the He bubble, decreases with He pressure. On the other hand, 
the transition frequency $\omega_{0}$ increases with the pressure, as discussed in Sec. 7.3. The radiative lifetime is given by the expression

$$
\frac{1}{\tau}=\frac{\omega_{0}^{3} e^{2} d^{2}}{3 \pi \epsilon_{0} \hbar c^{3}\left(2 J_{e}+1\right)},
$$

where $J_{e}=1 / 2$ is the total electronic angular momentum of the excited state.

The difference between the lifetime of the free Cs atom and a Cs atom embedded in a $\mathrm{He}$ matrix is mostly due to the so-called cavity effect $[181,134]$. The electromagnetic radiation of the atomic dipole in the bubble is partially reflected at the bubble interface and interacts with the dipole. This effect results in a redshift of the transition frequency discussed in Sec. 7.3 and also reduces the lifetime by approximately 3 ns. The observed decrease of $\tau$ with He pressure in the hcp phase is attributed to the enhancement of $\mathrm{Cs}^{*} \mathrm{He}_{N}$ exciplex formation due to the decrease of the bubble radius.

The redshift of the transition energy and the cavity effect were also considered by the authors of [153] to be responsible for the observed $20 \%$ increase of the lifetime of the $3^{1} P_{1}$ excited state of $\mathrm{Mg}$ atom embedded in He droplet.

\subsection{Lifetimes of $\mathrm{Cs}^{*} \mathrm{He}_{N}$ exciplexes}

First measurements of the lifetime of alkali-helium exciplexes were performed for $\mathrm{Na}^{*} \mathrm{He}$ formed on the surface of He nano-droplets [95] and later for $\mathrm{K}^{*} \mathrm{He}$ [97]. In both cases it was shown that the exciplex lifetime is longer than that of a corresponding excited state of a free alkali atom. The effect could be explained through Eq. 34, assuming that the transition dipole moment $d$ is not affected by the presence of the He atom, and using the emission wavelength $\omega_{0}$ determined from spectroscopic observations.

In solid He the measurements were performed for $\operatorname{Cs}\left(6 P_{1 / 2}\right) \mathrm{He}_{N}$ and $\operatorname{Cs}\left(6 P_{3 / 2}\right) \mathrm{He}_{2}$ [182] by observing the time-resolved fluorescence following a pulsed laser excitation. Due to the extremely large redshift of the fluorescence emitted by $\mathrm{Cs}\left(6 P_{1 / 2}\right) \mathrm{He}_{N}$, the corresponding lifetime obtained using Eq. 34 is $\approx 130$ ns. Indeed, in the experiment we have measured a value of $\tau=95 \mathrm{~ns}$, much larger than that of the $6 P_{1 / 2}$ state in the free Cs atom $\tau_{\text {free }}=$ 34.8 ns. However, this value is lower than expected. The discrepancy is attributed to the modification of the transition dipole moment. The interaction with the ring of He atoms results in a strong mixing of the $6 S$ and $5 D$ states. The effect has the same mechanism as the $S-D$ mixing by the deformed bubble discussed in Sec. 4.3, but is much stronger due to the small radius of the ring. Since the transition dipole moment between the $6 P$ and $5 D$ states is even larger than that of the $6 P-6 S$ transition, the admixture of the $5 D$ state to the ground state results in the enhancement of the latter transition.

The time-resolved fluorescence of $\operatorname{Cs}\left(6 P_{3 / 2}\right) \mathrm{He}_{2}$ yields a pulse shape that can not be distinguished from the exciting laser pulse with our current time-resolution. The corresponding lifetime must be around $1 \mathrm{~ns}$, or smaller. The origin of this quenching effect is not yet clear. The most probable explanation is that $\mathrm{Cs}\left(6 P_{3 / 2}\right) \mathrm{He}_{2}$ is a transient complex, quickly evolving into the terminal $\mathrm{Cs}\left(6 P_{1 / 2}\right) \mathrm{He}_{N}$ exciplex. A dissociation into an excited $\mathrm{Cs}\left(6 P_{1 / 2}\right)$ or Cs $\left(6 P_{3 / 2}\right)$ atom and two He atoms can be excluded due to the complete absence of Cs $D_{2}$ fluorescence and the relatively low intensity of the $D_{1}$ fluorescence. 


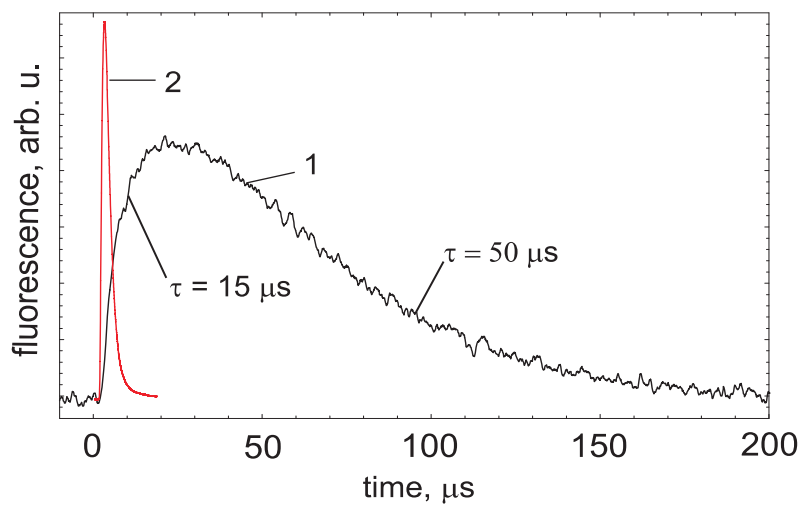

Fig. 25. Time resolved fluorescence of the $\mathrm{Rb}_{2}$ molecule in solid He (curve 1). Curve 2 shows the excitation laser pulse (actual pulseshape is not resolved.)

\subsection{Lifetime of the metastable $(1)^{3} \Pi$ states of $R b_{2}$ and $R b C s$}

The time-resolved molecular fluorescence of $\mathrm{Rb}_{2}$, excited by $\mathrm{OPO}$ pulses at $580 \mathrm{~nm}$ is shown in Fig. 25 [133]. Similar results were obtained for the RbCs and $\mathrm{Cs}_{2}$ molecules. We have found that the fluorescence pulse has a decay time of about $50 \mu \mathrm{s}$, much longer than the characteristic lifetime of any allowed electronic transition. This led us to assign the observed fluorescence to the forbidden triplet-singlet transition (as discussed in Sec. 7.6). The pulse shape of the excitation laser is also shown in Fig. 25. The fluorescence pulse has a finite rise time of $\tau \simeq 15 \mu$ s that is significantly longer than that of the laser.

The finite rise and decay times point to the formation of a state that does not exist in the free dimer. We assign [133] this behavior to the formation of a molecular exciplex state, from which the observed fluorescence emanates.

\section{Magnetic resonance experiments}

Most of today's knowledge about the properties of solid He matrix-isolated atoms comes from pure optical (absorption and fluorescence) studies. However, magnetic resonance experiments can yield valuable complementary information on the structure and dynamics of the atomic trapping sites. At first glance this looks surprising since ${ }^{4} \mathrm{He}$ has neither an electronic, nor an orbital nor a nuclear magnetic moment and does thus not couple to the spin of paramagnetic atoms in lowest order. Moreover, the successful description of optical spectra by the spherical bubble model in bcc ${ }^{4} \mathrm{He}$ matrices is a confirmation that spherically symmetric atoms, such as the alkalis, impose their spherical symmetry on the local trapping site. As a consequence one would expect that spin-dependent properties of the dopants should not be affected by spherical bubbles, so that such matrices should be an ideal environment for experiments that call for a long-lived spin polarization, such as high resolution magnetic resonance studies.

The search for a permanent electric dipole moment (EDM) of paramagnetic atom is such an application, and 15 years ago we proposed $[119,183]$ that solid helium might be an ideal matrix to search for an EDM in heavy paramagnetic atoms. With such an experiment in mind we started to develop the field of solid helium matrix isolation spectroscopy in the early 
1990's. We could demonstrate that, as expected, Cs and Rb atoms have indeed exceptionally long longitudinal and transverse spin relaxation times on the order of 1 second. This paved the way to a number of high resolution magnetic resonance experiments which we shall review in this section.

In contrast to conventional (ESR) magnetic resonance studies on atomic valence electrons which use magnetic fields of a few tenths of Tesla our experiments are carried out in fields of a few $\mu T$, a fraction of the earth magnetic field. In all magnetic resonance experiments we use the powerful technique of optically detected magnetic resonance (ODMR), in which resonant laser radiation is used both to polarize the sample and to detect alterations of the spin polarization. Such alterations may be induced by the magnetic resonance process proper, by external electric or magnetic fields and gradients thereof, or by matrix perturbations.

The effective transverse spin relaxation time of $\mathrm{Cs}$ in other inert-gas matrices is below 1 $\mu s$, five orders of magnitude shorter than the $T_{2}$ times obtained in solid ${ }^{4} \mathrm{He}$. Because of the long transverse spin relaxation times of $\mathrm{Cs}$ and $\mathrm{Rb}$ in bcc ${ }^{4} \mathrm{He}$ magnetic resonance lines in that matrix are exceptionally narrow.

\subsection{The technique of optically detected magnetic resonance (ODMR)}

Magnetic resonance experiments require the sample to be spin polarized and the magnetic resonance signal is proportional to the degree of longitudinal spin polarization $P_{z}$. In conventional ESR experiments (Sec. 10.2.5, 11.5) the atoms are polarized via the Boltzmann factor $e^{-\frac{\mu B}{k T}}$ that makes use of a strong external magnetic field and the low temperature of the sample.

The technique of optical pumping, developed in the 1950's for polarizing dilute atomic vapors can be applied to alkali atoms in condensed ${ }^{4} \mathrm{He}$ as shown first in [123] for superfluid matrices and later by us [184] for solid He matrices. When irradiating the sample exposed to a moderate magnetic field of a few $\mu T$ with circularly polarized resonance light angular momentum is transferred from the light beam to the atoms. This leads to a build-up of a vector spin polarization (orientation) $P_{z}=\left\langle J_{z}\right\rangle \propto\left\langle F_{z}\right\rangle_{F=4}-\left\langle F_{z}\right\rangle_{F=3}$ by the redistribution of populations among the ground state Zeeman sublevels [185]. This creation of polarization is counteracted by longitudinal relaxation. Because of the long $T_{1}$ times of alkalis in solid He the process is particularly efficient and can be achieved with moderate laser powers. Typically a few $\mathrm{mW}$ of an unfocussed laser beam of $1 \mathrm{~mm}$ diameter are sufficient to create a significant degree of spin polarization in $\mathrm{Cs}$ in bcc He. We note that the required pumping intensity in helium is approximately 4 orders of magnitude larger than the intensity needed to pump an atomic vapor, the reason being the large homogeneous width of the optical transition (a few $\mathrm{THz}$ ) which reduces the peak absorption cross section and hence the pumping efficiency with respect to atomic gases with a Doppler-broadened absorption line of a few $100 \mathrm{MHz}$. Since absorption on the $D_{2}$ line $\left(n S_{1 / 2} \rightarrow n P_{3 / 2}\right.$ transition $)$ in Cs produces almost no fluorescence (see Sec. 7.3) all pumping experiments to date were performed using excitation on the $D_{1}$ transition $\left(n S_{1 / 2} \rightarrow n P_{1 / 2}\right)$ with solid state diode lasers or a $\mathrm{Ti}: \mathrm{Al}_{2} \mathrm{O}_{3}$ laser.

Because of the large optical linewidth one cannot resolve the hyperfine structure of the ground and excited states in the excitation spectrum. In that case all hyperfine components $F \rightarrow F^{\prime}$ ( $F$ being the total atomic angular momentum resulting from the coupling 
of the electronic spin, $S$, and the nuclear spin, $I$ ) are excited simultaneously according to the selection rule $\Delta F=0, \pm 1$, similarly to the pumping of an atomic vapor by a spectrally broad light source, such as discharge lamp. Nevertheless, populations will be redistributed between Zeeman and hyperfine levels by the pumping process. It is well known that atomic vapors pumped on an isolated hyperfine transition lead to the creation of an atomic alignment (quadrupole tensor polarization) $A_{z z} \propto\left\langle 3 F_{z}^{2}-F^{\prime} 2\right\rangle$ [186]. Because of the unresolved hyperfine structure of the optical transition in solid He, however, it is not possible to create a ground state alignment by optical pumping with linearly polarized light.

The optical absorption coefficient of an atomic medium depends on the orientation of its spin polarization $\vec{P}$ with respect to the light polarization $\widehat{\varepsilon}$. Pumping atoms on the $D_{1}$ transition with $\varepsilon_{ \pm}$circularly polarized light ultimately puts all atoms in the state $\mid F=I+J, M=$ $\pm(I+J)\rangle$. This state cannot be excited by radiation of the same polarization as the one that has produced it. The state is therefore referred to as dark state and pumping to this state is characterized by a strong reduction of the fluorescence intensity. Any effect which changes the magnitude or the orientation of the polarization, such as a magnetic resonance transition, leads to a variation of the absorbed and scattered light intensities. The powerful technique which combines the creation of spin polarization by optical pumping, magnetic resonance, and the optical detection of the latter is known as optically detected magnetic resonance (ODMR). Although the three processes (preparation, magnetic resonance, detection) occur simultaneously they can often be described theoretically as three time-sequential processes [185].

Alkali-doped He crystals are optically thin with extinction ratios below $10^{-4}$. Except for a single experiment in which we have recorded absorption using a special technique (described in section 9.2 and [187]) all magnetic resonance experiments relied on fluorescence detection.

\subsection{Optical pumping and magnetic resonance experiments}

The first experimental demonstration of optical pumping of alkali atoms in condensed helium was performed with ${ }^{85} \mathrm{Rb},{ }^{87} \mathrm{Rb}$, and ${ }^{133} \mathrm{Cs}$ in superfluid helium [123]. In those experiments, spin polarization was created by optical pumping of rubidium or cesium atoms implanted by laser sputtering into liquid helium.

In 1995 we showed that efficient optical pumping is also possible with alkali atoms embedded in the cubic phase of solid ${ }^{4} \mathrm{He}$ [184] and, less efficiently, in the anisotropic hexagonal phase of the matrix $[188,70]$. Those experiments proved our original assumption that the spin polarization of alkali atoms in solid helium may be very long-lived. A subsequent detailed study of the optical pumping process in the bcc and in the hcp phase [187] revealed that optical pumping in Cs is of the repopulation type, in which the spin polarization in the excited $P_{1 / 2}$ state - populated for a few 10 ns during the pumping cycle - is not affected by the interaction with the helium matrix, while it is known that high pressure He gas completely quenches the excited state polarization. The collision rate of the bubble interface atoms with the Cs atom is so high that the spin-orbit interaction following a collision-induced local deformation of the Cs atom has no time to efficiently disorient the Cs spin before the next collision (from another direction) occurs. In Rb, on the other hand, exciplex formation is the main deexcitation channel of the $\mathrm{P}_{1 / 2}$ state (cf section 7.4), so that optical pumping of the 

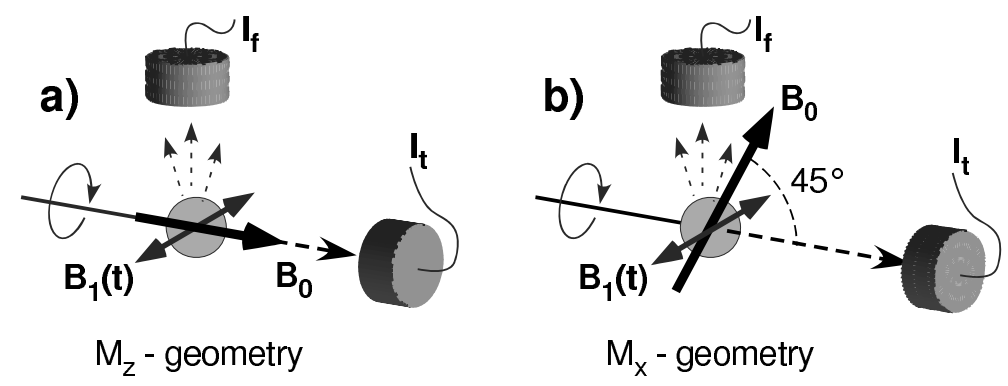

Fig. 26. Optically detected magnetic resonance (ODMR) spectroscopy can be performed in 2 different geometries which both use a static $\left(B_{0}\right)$ and an oscillating ( $B_{1}$, rf or microwave) magnetic field: a) In the $M_{z}$ geometry the magnetic field is oriented along the light propagation and the fluorescence (or transmitted) intensity shows a resonant DC change when the Larmor frequency coincides with the oscillating field frequency; b) in the $\mathrm{M}_{x}$ geometry the the magnetic field is oriented at $45^{\circ}$ with respect to the light propagation and the fluorescence (or transmitted) intensity is modulated at the frequency of the oscillating field.

$\mathrm{Rb}$ isotopes is of the depopulation type. As shown in [187] depopulation and repopulation pumping lead to specific redistributions of the sublevel populations in the two hyperfine ground state levels, an effect, which can be used for the experimental distinction of the two mechanisms.

Optical pumping of cesium in the anisotropic hcp phase of helium is much less efficient than in the isotropic bcc phase. We could show that the degree of spin polarization achievable in hcp ${ }^{4} \mathrm{He}$ depends strongly on the value of the magnetic holding field $\mathrm{B}_{0}$, while in bcc it was found to be independent of $\mathrm{B}_{0}$ [187]. This effect is one of the multiple manifestions of bubble deformations discussed in Sec. 9.8.

As mentioned above, Kinoshita et al. [123] were the first to observe magnetic resonance in alkalis implanted in superfluid helium using the $M_{z}$ variant (Fig. 26.a) of ODMR (Sec. 9.1 . The longer relaxation time obtained in the cubic phase of solid helium have allowed us to observe magnetic resonance lines in such matrices with a width (HWHM) of only $10 \mathrm{~Hz}$ [189]. We took advantage of these narrow lines to build an optically pumped magnetometer in the $M_{x}$ geometry [189] and were able to demonstrate a magnetometric sensitivity of $2.6 \mathrm{pT}$ for an integration time of 1 second.

Because of exciplex formation the fluorescence intensity of the $\mathrm{Rb} D_{1}$ line in superfluid helium is strongly quenched when the helium pressure is increased and it was long believed that $\mathrm{Rb}$ would not fluoresce at all in solid helium. Recently we could observe a faint fluorescence from $\mathrm{Rb}$ in solid helium [113]. Kinoshita et al. [123] were able to observe ODMR signals of $\mathrm{Rb}$ in superfluid He using fluorescence detection, because of the relatively strong fluorescence in that phase. In the days when it was believed that Rb would not fluoresce in solid He we were nevertheless able to detect magnetic resonance using absorption spectroscopy. The typical resonant optical absorption of alkali-doped solid helium samples is on the order of $10^{-5}-10^{-4}$, which makes its detection difficult. Because of the limited tuning range of our laser sources we were not able to isolate the atomic absorption signal from the background by a simple scan of the absorption line. Our successful detection of magnetic resonance in Rb relied on the $M_{x}$ variant (Fig. 26) of ODMR [190], in which the magnetic resonance process induces a modulation of the transmitted intensity at the rf frequency. The use of lock-in detection yielded a signal/noise ratio large enough to identify magnetic resonance signals from $\mathrm{Rb}$ in bcc ${ }^{4} \mathrm{He}$ in the transmitted laser intensity [191]. In those ex- 

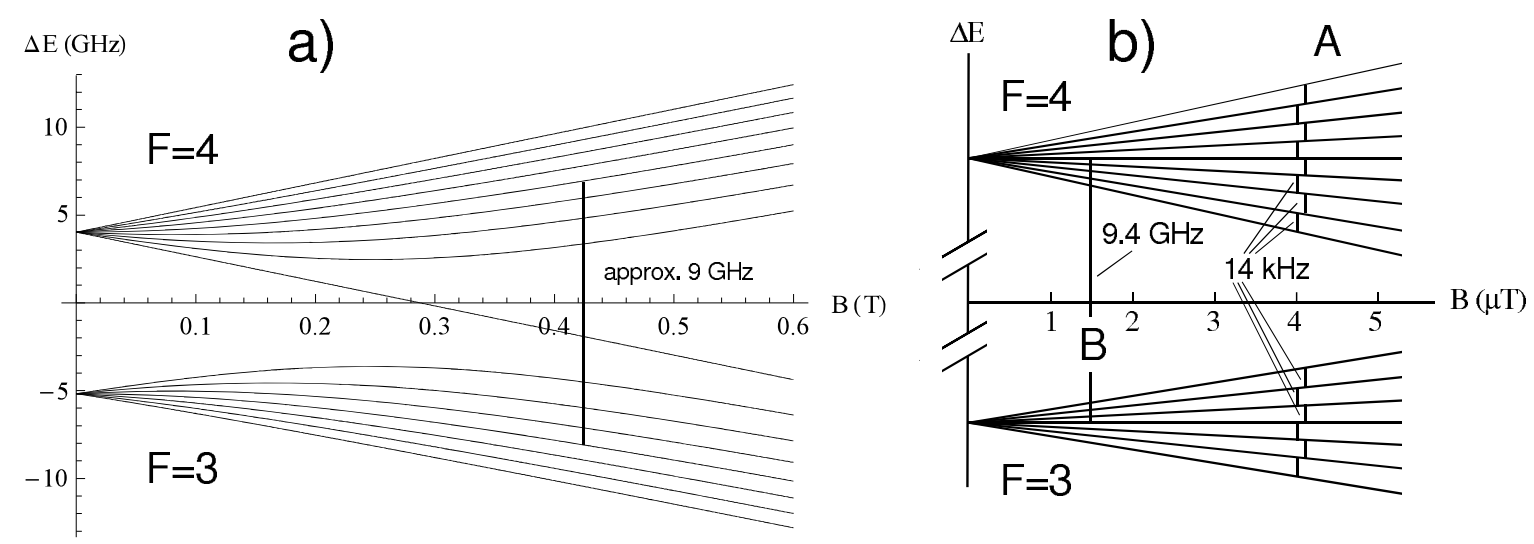

Fig. 27. Hyperfine-Zeeman structure of the Cs atom. a) Conventional ESR experiments are performed in magnetic fields of 0.1 to several T. b) Magnetic fields in ODMR studies are typically performed in $\mu T$ fields. The arrows mark typical frequencies of intra- and inter-multiplet transitions explored with Cs in solid ${ }^{4} \mathrm{He}$.

periments we have studied the Landé $g$-factors of ${ }^{85} \mathrm{Rb},{ }^{87} \mathrm{Rb}$, and ${ }^{133} \mathrm{Cs}$ and found that the helium matrix does not affect the electronic $g_{J}$-factors at a level of $2 \cdot 10^{-4}$, and that the nuclear $g_{I}$ factors coincide at least at a level of $10 \%$ with those of the free atoms [191]. That study also revealed that, because of exciplex formation the optical pumping process in $\mathrm{Rb}$ is of the depopulation type and it should therefore in principle be possible to monitor the ground state spin polarization via exciplex fluorescence.

\subsection{Zeeman structure}

Conventional electron spin resonance (ESR) experiments use strong magnetic fields in the region of $100 \mathrm{mT}$ to several Tesla with electron spin flip transition frequencies around 10 GHz. Fig. 27(a) shows the typical hyperfine Zeeman pattern (Breit-Rabi diagram) of Cs in that field region. In sections 10.2 .5 and 11.5 below we will review some ESR experiments carried out in impurity-stabilized He, and on matrix-isolated paramagnetic atoms, respectively.

All magnetic resonance studies involving optical detection (ODMR) on alkalis in condensed helium were performed in magnetic fields of a few $\mu \mathrm{T}$, lower than the earth magnetic field, for which the coupling between the electronic and nuclear spins is much stronger than their hyperfine interaction with the magnetic field. The hyperfine-Zeeman structure of the Cs ground state is shown in Fig. 27(b) in which two types of magnetic dipole transitions are indicated : intramultiplet transitions between the Zeeman sublevels of the same hyperfine level $F$, and intermultiplet (hyperfine) transitions between $M_{F}$ levels of different hyperfine $F$ levels.

\subsection{Magnetic resonance spectra and multi-photon transitions}

Magnetic resonance transitions are magnetic dipole transitions which obey the selection rule $\Delta M= \pm 1$ reflecting the absorption of one $\sigma_{ \pm}$circularly polarized rf photon in a transition between adjacent Zeeman sublevels (Fig. 29.a). In small magnetic fields (linear Zeeman 

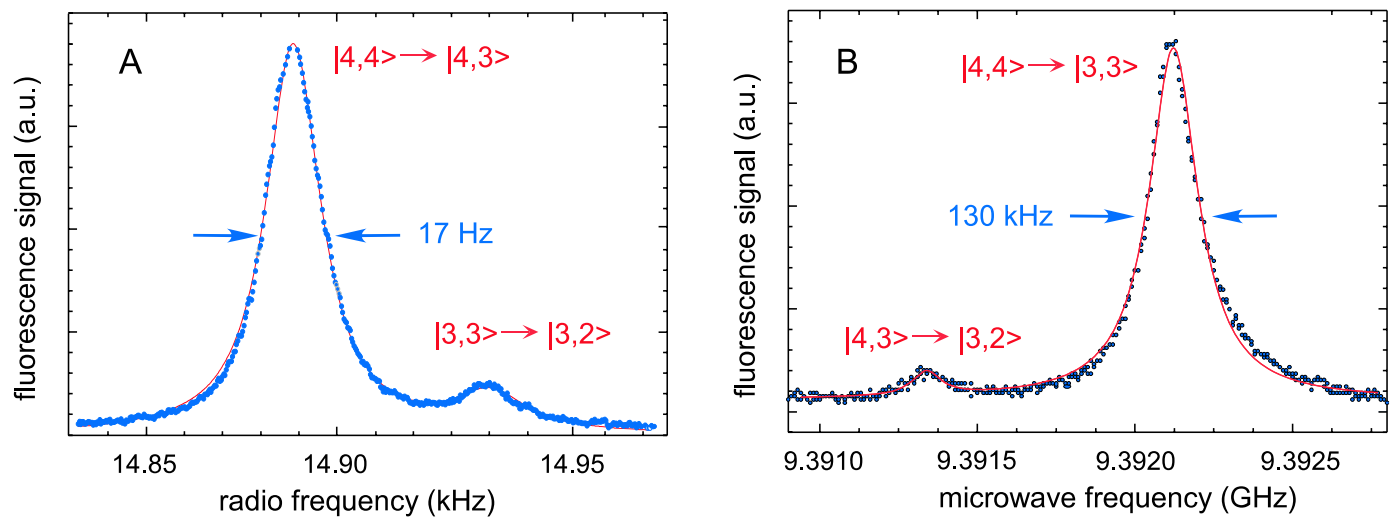

Fig. 28. Intramultiplet (A) and intermultiplet (B) magnetic resonance spectra of Cs in bcc ${ }^{4} \mathrm{He}$. A and B refer to the transitions shown in Fig. 27. Note the difference of the linewidths in the two cases.

regime) the level spacings are all equal to the Larmor frequency $\omega_{L} \propto g_{F} B$, where $g_{F}$ is the Landé $g$-factor. The resonance frequencies are all degenerate (independent of $M$ ) and the magnetic resonance spectrum of the intra-multiplet transitions $\left|n S_{1 / 2}, F, M\right\rangle \rightarrow$ $\left|n S_{1 / 2}, F, M \pm 1\right\rangle$ consists of a single line. Because of the nuclear magnetism, however, the moduli $\left|g_{F}\right|$ of the effective Landé-factors of the two ground state hyperfine levels $F_{ \pm}=$ $I \pm J=I \pm 1 / 2$

$$
\begin{aligned}
& g_{F_{+}}=g_{F=I+1 / 2}=+\frac{g_{J}}{2 I+1}-\frac{2 I}{2 I+1} g_{I} \\
& g_{F_{-}}=g_{F=I-1 / 2}=-\frac{g_{J}}{2 I+1}-\frac{2 I+2}{2 I+1} g_{I}
\end{aligned}
$$

differ by $\Delta g_{F}=\left|g_{F_{-}}\right|-\left|g_{F_{+}}\right|=2 g_{I}$, leading to a relative splitting

$$
\frac{\Delta \nu_{r f}}{\left\langle\nu_{r f}\right\rangle}=(4 I+2) \frac{g_{I}}{g_{J}} \approx 3.2 \cdot 10^{-3}
$$

of the magnetic resonance in the ${ }^{133} \mathrm{Cs}(\mathrm{I}=7 / 2)$ ground state.

Because of the (optically) unresolved hyperfine structure the laser interacts simultaneously with both hyperfine states so that the two transitions can be observed in a single scan of the rf frequency (Fig.28.A). When the intensity of the radiofrequency radiation driving the resonances is increased the lines broaden and saturate. With sufficient rf intensity multiphoton transitions will then become possible. A first class of such resonances are transitions between states differing by $\Delta M=2$, in which two photons (of the same helicity) are absorbed simultaneously (Fig. 28.b). In the level $F$ there are $2 F$ distinct one-photon transitions and $2 F-1$ distinct two-photon transitions. As the rf intensity is further increased higher order transitions involving the simultaneous absorption of $n$ photons between states obeying $\Delta M=n$ become possible, until finally a single transition $(n=2 F+1)$-photon transition coupling the states $\left|n S_{1 / 2}, F, M=+F\right\rangle$ and $\left|n S_{1 / 2}, F, M=-F\right\rangle$ appears. In the linear Zeeman regime all those transitions are degenerate and cannot be distinguished in the spectrum. In fact, saturation and power broadening of the isolated magnetic resonance line may be interpreted in a perturbative approach as being the superposition of all those lines. A simple way to resolve all the individual multiphoton processes is the use of the quadratic Zeeman shift of 


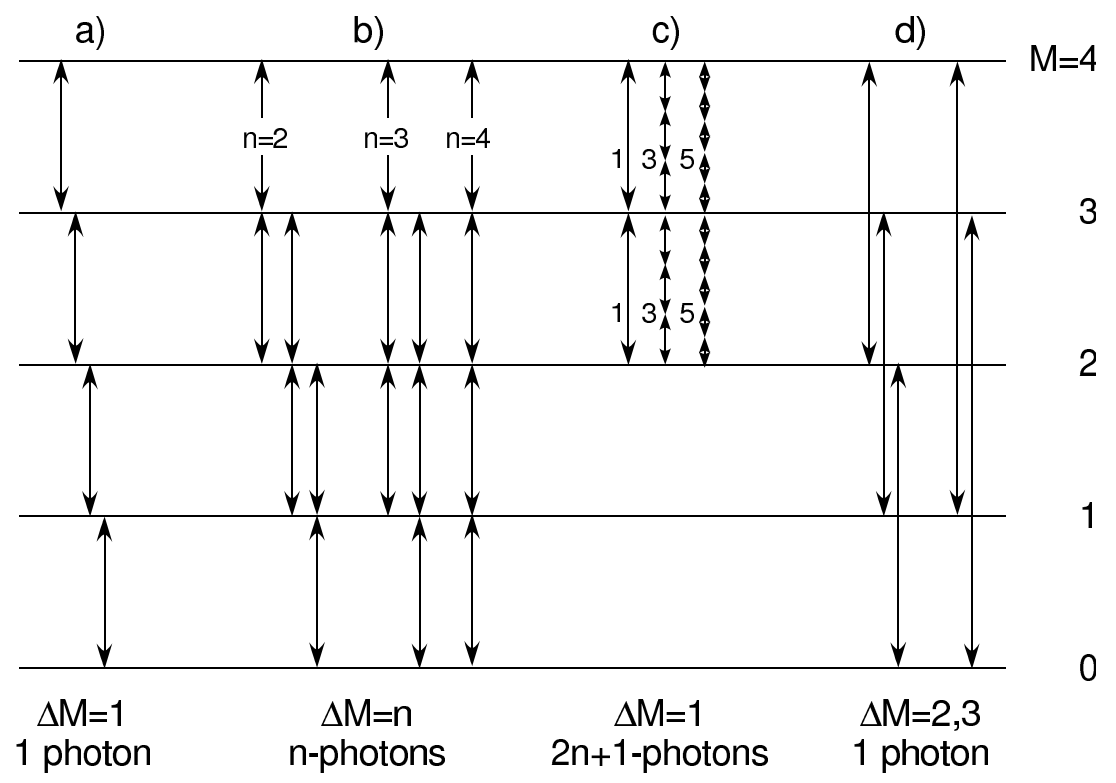

Fig. 29. (a) Possible transitions involving absorption of $n \operatorname{rf}$ photons between the Zeeman sublevels of the $F=4$ ground state of the Cs atom, in a magnetic field which produces a linear and quadratic Zeeman shift. (b) Magnetic resonance spectra showing the multiphoton transitions of (a). The rf power increases from the bottom to the top.

the sublevel energies that appears in the Breit-Rabi diagram (Fig. 27) when increasing the magnetic field strength beyond the linear Zeeman regime. The long spin relaxation times and the narrow magnetic resonance lines of cesium in bcc solid helium make this sample well suited for the study of such processes. In an experimental study [192] we have indeed observed all multi-photon processes in the $F=4$ hyperfine ground state up to the process of simultaneous absorption of $8 \mathrm{rf}$ photons. In a subsequent detailed theoretical analysis we have investigated the influence of different relaxation mechanisms on the shape of those multi-photon spectra [193].

The magnetic dipole selection rules also allow $\Delta M=1$ transitions between adjacent sublevels through the absorption of $2 n+1$ photons (Fig. 29.c). The angular momentum selection rule $\Delta M=1$ is fulfilled if the ensemble of photons is composed of $n+1$ photons of polarization $\sigma_{ \pm}$and $n$ photons of polarization $\sigma_{\mp}$, respectively. Fig. 30 shows an example of such a multiphoton spectrum, in which up to $n=9$ photons are absorbed simultaneously.

The selection rule $\Delta M=1$ is a consequence of the symmetry imposed by the external magnetic field. It no longer holds if this cylindrical symmetry is broken by an additional non spherical perturbation, such as the Stark interaction in a transverse electric field, or the interaction with a (tilted) non-spherical atomic bubble, in which case $\Delta M=2,3, \ldots$ transitions become allowed. Fig. 34 shows an example of such "forbidden" lines.

\subsection{Hyperfine transitions}

The optical pumping process produces not only population imbalances between the Zeeman sublevels in a given hyperfine state $F$, but also a population imbalance between the two hyperfine levels $F_{ \pm}$. Besides driving $M$-changing intramultiplet (Zeeman) transitions within a given hyperfine multiplet (transitions A in Fig.27.b), one can therefore also also 


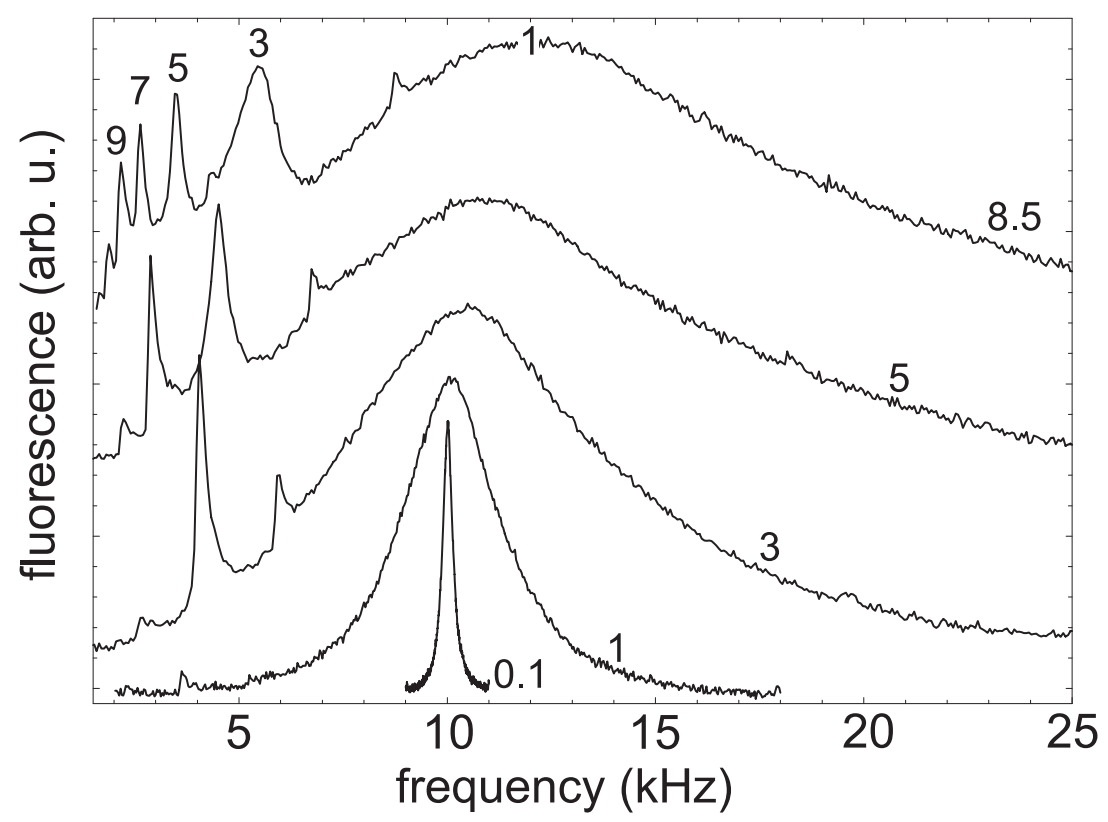

Fig. 30. Magnetic resonance spectra showing multiphoton transitions between adjacent Zeeman levels. Numbers on the right side of each curve indicate the relative rf power in arbitrary units, numbers on top of the figure show the number of absorbed rf photons.

drive $F$-changing intermultiplet (hyperfine) transitions (transitions B in Fig.27.b). In low magnetic fields the corresponding resonance frequency (clock transition frequency) is on the order of $9 \mathrm{GHz}$. We have studied the hyperfine transition in cesium atoms implanted in bcc and hcp ${ }^{4} \mathrm{He}$ [73]. In the bcc phase the transition frequency is blue shifted by approximately $200 \mathrm{MHz}$ with respect to the transition in the free atom (see Fig. 33C). The He matrix compresses the wave function of the valence electron, so that $\left|\Psi_{6 S_{1 / 2}}(0)\right|^{2}$ and thus the hyperfine coupling constant of the Fermi contact interaction increase, hence the matrix-induced blue shift of the transition frequency. We also found that the shift increases with He pressure at a rate of $\sim 1.4 \mathrm{MHz} /$ bar [73]. The quantitative value of the shift and its pressure dependence can be predicted in a satisfactory way by the bubble model $[1,21,48]$. Surprisingly, the linewidth $(100 \mathrm{kHz})$ of the hyperfine transition in bcc ${ }^{4} \mathrm{He}$ is approximately 4 orders of magnitude larger than the width (10-20 Hz) of the intramultiplet Zeeman transitions. We believe that dynamic bubble deformations (quadrupole oscillations) are responsible for the large linewidth of the hyperfine transition (cf. Sec.9.8).

Since the He density is larger in the hcp phase one would expect a corresponding increase of the blue shift of the hyperfine resonance by $2 \mathrm{MHz}$. Experiments (Fig. 33C) show, however, that the clock transition shifts to the red by $\approx 7 \mathrm{MHz}$ when going from bcc to hcp [70]. The red shift may be explained qualitatively in terms of (static) bubble deformations as discussed in Sec. 4.3.

\subsection{Relaxation times}

Alkali atoms in condensed ${ }^{4} \mathrm{He}$ were found to have very long longitudinal $\left(\mathrm{T}_{1}\right)$ and transverse $\left(\mathrm{T}_{2}\right)$ relaxation times. The longitudinal relaxation time $T_{1}$ characterizes the rate at 
which the longitudinal polarization $\mathrm{P}_{z}$, i.e., the polarization component along the magnetic field relaxes. The original optical pumping experiments [123] on Rb and $\mathrm{Cs}$ in superfluid helium did not determine the corresponding $\mathrm{T}_{1}$ time. It was only recently that a careful determination of Cs in HeII was obtained by Furukawa et al. [194] who determined a lower bound of $T_{1}=2.24(19) \mathrm{s}$ in a field of $500 \mu \mathrm{T}$. This relaxation time is approximately two times longer than the $\mathrm{T}_{1}$ time in solid ${ }^{4} \mathrm{He}$ which we have determined to be $\mathrm{T}_{1} \approx 1 \mathrm{~s}$ in external magnetic fields ranging from $10 \mathrm{nT}$ to $100 \mu \mathrm{T}$ [187]. These relaxation times are longer than the $T_{1}$ times observed in atomic vapor cells in which special surface coatings or the addition of inert buffer gases are used to prevent depolarizing collisions with the cell walls. Hatakeyama et al. [195] have measured a $\mathrm{T}_{1}$ time of $96 \pm 15 \mathrm{~s}$ in Cs atoms injected into cryogenic He gas contained in a cell whose walls were covered with a superfluid He film.

The widths of magnetic resonance and level crossing signals are determined by the transverse spin relaxation time $T_{2}$, i.e., by the rate at which the polarization components $\mathrm{P}_{x, y}$ perpendicular to the magnetic field relax. There is no a priori reason why the $\mathrm{T}_{2}$ times of alkali atoms in condensed helium should differ significantly from the $T_{1}$ times. In principle the transverse relaxation time can be inferred from the withs of magnetic resonance lines, which may, however, be substantially affected by light and/or rf power broadening and magnetic field gradients. The first experiments that reported magnetic resonance on $\mathrm{Rb}$ and $\mathrm{Cs}$ atoms in superfluid helium [123] had to use an excessively large intensity of the rf field in order to overcome signal loss due to thermal convection in the liquid matrix. The observed magnetic resonance linewidths were on the order of $50 \mu \mathrm{T}$ which corresponds to a coherence relaxation time on the order of $1 \mu \mathrm{s}$. Solid helium matrices avoid this complication since the atoms are immobilized and the magnetic resonance lines are significantly narrower in such matrices.

In our 1995 recordings of longitudinal/transverse level crossing signals (ground state Hanle effect) and of magnetic resonance lines [184] we observed linewidths on the order of $300 \mathrm{nT}$, corresponding to effective $T_{2}$ times of $150 \mu \mathrm{s}$. Contributions from optical power broadening were subsequently eliminated by using the technique of free induction decay in the dark, which yielded a lower limit $T_{2}>$ of 108(3) ms [189], one order of magnitude below the $T_{1}$ time. Under the experimental conditions of that experiment a magnetic field inhomogeneity of $\approx 10^{-7}$ would yield a comparable $\mathrm{T}_{2}$ time and is probably responsible for $\mathrm{T}_{2}$ being smaller than $\mathrm{T}_{1}$. Unpublished spin echo experiments indicate that $\mathrm{T}_{2}$ is indeed larger than $100 \mathrm{~ms}$.

\subsection{The Stark effect of $\mathrm{Cs}$ in $b c c{ }^{4} \mathrm{He}$}

The measurement of the tensor polarizability of Cs atoms isolated in solid He is another application that makes use of the narrow linewidths found in intramultiplet magnetic resonance transitions. In an external electric field the energy of a magnetic sublevel $\left|6 S_{1 / 2}, F, M\right\rangle$ in an alkali atom shifts according to

$$
\Delta E\left(6 S_{1 / 2}, F, M\right)=-\frac{1}{2} \alpha\left(6 S_{1 / 2}, F, M\right) \mathbb{E}^{2}
$$

where the polarizability $\alpha\left(6 S_{1 / 2}, F, M\right)$ is given by 

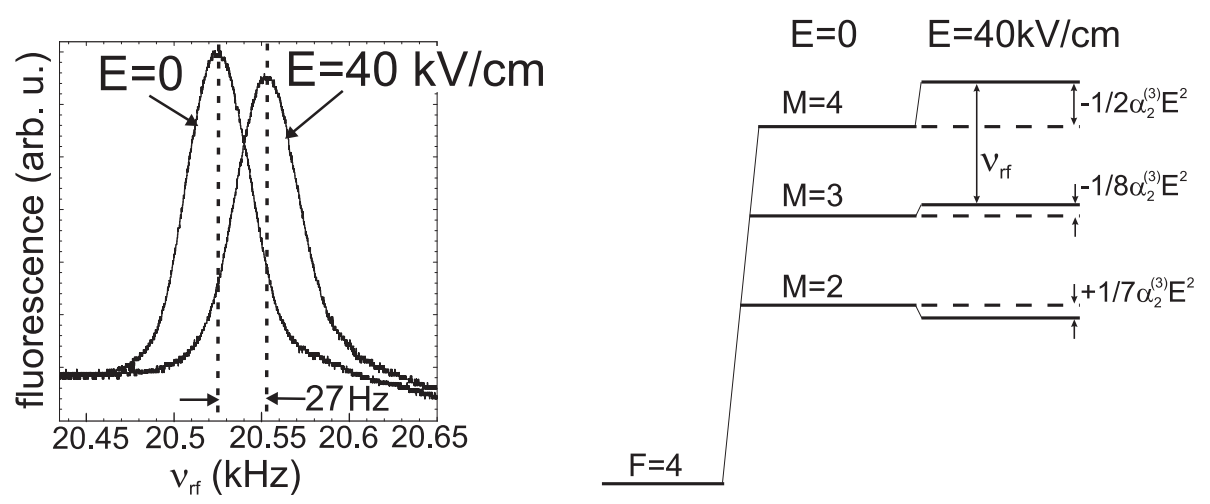

Fig. 31. Left: quadratic Stark shift of the magnetic resonance frequency in a magnetic field of $6 \mu \mathrm{T}$ recorded with and without electric field. Right: energies of Zeeman sublevels without and with electric field.

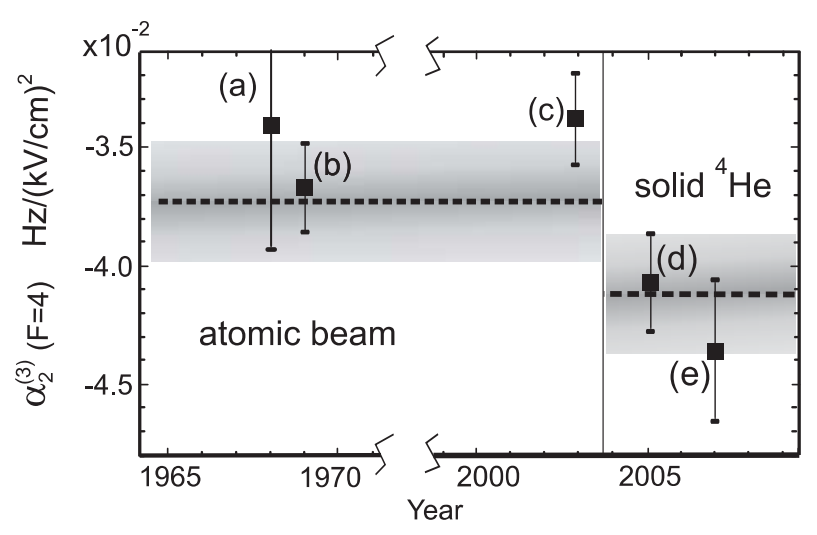

Fig. 32. Comparison of experimental values (dots) and theoretical predictions (dotted line) of the forbidden tensor polarizability of free Cs atoms (left) and of Cs atoms embedded in $\mathrm{bcc}^{4} \mathrm{He}$. The shaded bands mark the uncertainty of the theoretical results. Experimental results of (a) Carrico et al. [196], (b) Gould et al. [197], (c) Ospelkaus et al. [198], and (d, e) Ulzega et al. [199].

$$
\alpha\left(6 S_{1 / 2}, F, M\right)=\alpha_{0}^{(2)}+\alpha_{0}^{(3)}(F)+\alpha_{2}^{(3)}(F) \frac{3 M^{2}-F(F+1)}{I(2 I+1)}
$$

In the last equation the superscripts refers to the order of perturbation theory in which the polarizabilities appear, while the subscripts describe their rotational symmetry ( 0 for spherical and 2 for second rank tensor). The second order scalar polarizability, $\alpha_{0}^{(2)}$, is the leading term, followed by the $F$-dependent third order scalar polarizability, $\alpha_{0}^{(3)}$, and the third order tensor polarizability $\alpha_{0}^{(3)}$ which induces $F$ - and $M$-dependent shifts. It is to be noted that the last term induces shifts which are 7 orders of magnitude smaller than the leading term. Such shifts may, however, be isolated du to their explicit $M$-dependence. Since the energies of the adjacent states $|F, M\rangle$ and $|F, M \pm 1\rangle$ experience different Stark shifts the magnetic resonance frequency will be shifted by an amount which is proportional to $\alpha_{2}^{(3)}(F) \mathbb{E}^{2}$. Fig. 31 shows an experimental example of such an electric field-induced shift which has allowed us to infer the tensor polarizability of Cs atoms in bcc ${ }^{4} \mathrm{He}[200,199]$. As a result we find $\alpha_{2}^{(3)}(F=4)=-4.11 \cdot 10^{-2} \mathrm{~Hz} /(\mathrm{kV} / \mathrm{cm})^{2}$. The modulus of the tensor polarizability is found to be $8-10 \%$ larger than the tensor polarizability of the free Cs atom.

We have also revised the theoretical third order calculation of the tensor polarizability and have identified off-diagonal hyperfine mixing terms which were not taken into account 
in earlier calculations [200,72]. This has allowed us to finally settle a 40 year old discrepancy between experimental and theoretical values of $\alpha_{2}^{(3)}$. Recently we have extended those calculations to include the effect of spherical bubbles in He [201] and also find a good agreement with experiment. The experimental values for the free Cs atom and Cs in bcc ${ }^{4} \mathrm{He}$ are shown in Fig. 32. We have also performed a direct measurement of the difference between the tensor polarizabilities in the $F=3$ and $F=4$ hyperfine states [72]. Here again we find good agreement with our predictions, with disagree by a sign error from results obtained earlier (see discussion in [72]).

\subsection{Matrix effects in ODMR spectra}

The bubble model described in Sec. 3 is quite successful for describing optical spectra in spherical bubbles. The model assumes that the helium matrix is a continuous medium and that the bubble interface has a spherical shape. In Sec. 4 we argued that the spherical symmetry of the bubble is broken in (bcc and hcp) solid helium due to the anisotropic elastic properties of the corresponding crystalline structures. One therefore expects that specific effects associated with such deformations should appear in the optical and magnetic resonance spectra as one crosses the liquid-bcc and bcc-hcp phase boundaries. However, such phase transitions are also accompanied by a discontinuous change of the He density and it is often not possible to extract the pure deformation effect (if any) in an unambiguous way. Moreover, optical transitions involve two atomic states with different orbital symmetries and spatial extensions and deformed bubble effects appear only in a differential way. Magnetic resonance transitions, on the other hand occur within a state of given orbital momentum which simplifies the theoretical analysis of bubble deformation effects.

Deformed bubbles are characterized by a specific system of principal deformation axes. Since we have good reasons to believe that our matrices are poly-crystalline (a consequence of the brute force implantation technique) these axes have randomly distributed orientations and the spin of each trapped atom will couple to the deformation axis of its local anisotropic bubble. As shown in $[70,3]$ this coupling affects the longitudinal spin relaxation time $T_{1}$ since only the projection of the spin along the bubble axis is a conserved quantity. In case of large deformations the spin-bubble coupling competes with the spin-magnetic field coupling and one expects the $T_{1}$ time to become magnetic field dependent. In bcc we found that $T_{1}$ of the Cs ground state is independent of the magnitude of an externally applied magnetic field (varied in the range of $10 \mathrm{nT}-100 \mu \mathrm{T}$ ), while in hcp we observed a pronounced magnetic field dependence. From this one concludes that the deformations in the cubic phase are much less pronounced than in the hexagonal phase. In the following we will describe several other observations which can be traced back to bubble deformation, and from which a preliminary analysis has allowed us to infer the degree of bubble deformation.

\subsubsection{Zeeman transitions}

Intramultiplet magnetic resonance experiments on $\mathrm{Cs}$ and $\mathrm{Rb}$ in the bcc phase of ${ }^{4} \mathrm{He}$ yield sharp resonance lines with widths on the order of 10-20 Hz (Fig. 28.A) which show no asymmetries or line splitting that would be a signature of a static bubble deformation. One can thus conclude that the $L=4$ type of bubble deformation that one expects in the 
bcc phase (section 4.3) do not yield a differential level shift at the level of a few Hz. This situation changes dramatically when passing from the bcc to the hcp phase. In Fig. 33.A we compare magnetic resonance spectra recorded in both phases under similar conditions. The very narrow (single) magnetic resonance line observed with Cs in the bcc phase transforms into an extremely broad spectrum upon the bcc-hcp phase transition. The hcp spectrum is more than three orders of magnitude larger than the bcc spectrum. It consists of three equally spaced main components with a rich substructure that is probably due to a combination of forbidden transitions becoming allowed because of the broken symmetry in hep and the deformed matrix induce $M^{2}$ dependent level shifts. A detailed analysis of this particular spectrum requires a diagonalization of the combined Zeeman-deformed bubble Hamiltonian that takes the random orientation of the deformation axes (polycrystalline sample) with respect to the external magnetic field into account. Such an analysis has not been carried out to date.

\subsubsection{Zero field magnetic resonance spectrum}

In section 4.3 we have shown that the assumption of a quadrupolar bubble deformation in hcp leads to magnetic sublevel shift proportional to $M^{2}$. As a result one expects that the Zeeman degeneracy should be lifted even when no external magnetic field is applied. This effect was demonstrated in a convincing way by our observation [70] of a magnetic resonance spectrum in zero magnetic field (ZFS=zero field spectrum) as shown in Fig. 33.B. The main features of the spectrum can be understood as resulting from a quadrupolar $M^{2}$ dependent shift of the level energies, as shown in the insert. According to this interpretation the largest frequency line near $10 \mathrm{kHz}$ can be assigned to the $\left|6 S_{1 / 2}, 4,4\right\rangle \rightarrow\left|6 S_{1 / 2}, 4,3\right\rangle$ transition. This typical coupling strength of of $10 \mathrm{kHz}$ also manifests itself as a typical depolarization frequency in the magnetic field dependence of the longitudinal spin relaxation time $T_{1}$ on the magnitude of an externally applied magnetic field [3]. Using Eq. 29 one can obtain the $S-D$ mixing coefficient from the zero field frequency of the $\Delta \nu_{4 \rightarrow 3}^{\text {hcp }}$ transition:

$$
\varepsilon_{6 S, 5 D}=\sqrt{\frac{100}{21} \frac{\Delta \nu_{4 \rightarrow 3}^{\mathrm{hcp}}}{A_{\mathrm{hf}}\left(5 D_{3 / 2}\right)-A_{\mathrm{hf}}\left(5 D_{5 / 2}\right)}},
$$

where we have assumed that only the lowest lying $5 D_{J}$ states contribute to the sum in (40). We have calculated the hyperfine coupling constants of the $5 D_{J}$ states using Schrödinger wave functions of $\mathrm{Cs}$ in a spherical bubble (Sec. 3.4) and find $A_{\mathrm{hf}}\left(5 D_{3 / 2}\right)=-48.4 \mathrm{MHz}$ and $A_{\mathrm{hf}}\left(5 D_{5 / 2}\right)=17.8 \mathrm{MHz}$, to be compared to the experimental values in the free atom $A_{\mathrm{hf}}\left(5 D_{3 / 2,5 / 2}\right)=-41.9$ and $16.5 \mathrm{MHz}$, respectively. This yields a mixing coefficient $\varepsilon_{6 S, 5 D}=$ $2.7 \%$.

Zero-field magnetic resonance spectra are well known from atoms with a nuclear electric quadrupole moment implanted in uniaxial crystals, where the coupling of the quadrupole moment to local electric field gradients leads to a similar level shift [202]. The coupling frequencies encountered in those cases are usually in the $\mathrm{MHz}$ range, which illustrates the extreme weakness $\left(\approx 3 \cdot 10^{-7} \mathrm{~cm}^{-1}\right)$ of the anisotropy-induced coupling discussed here. 

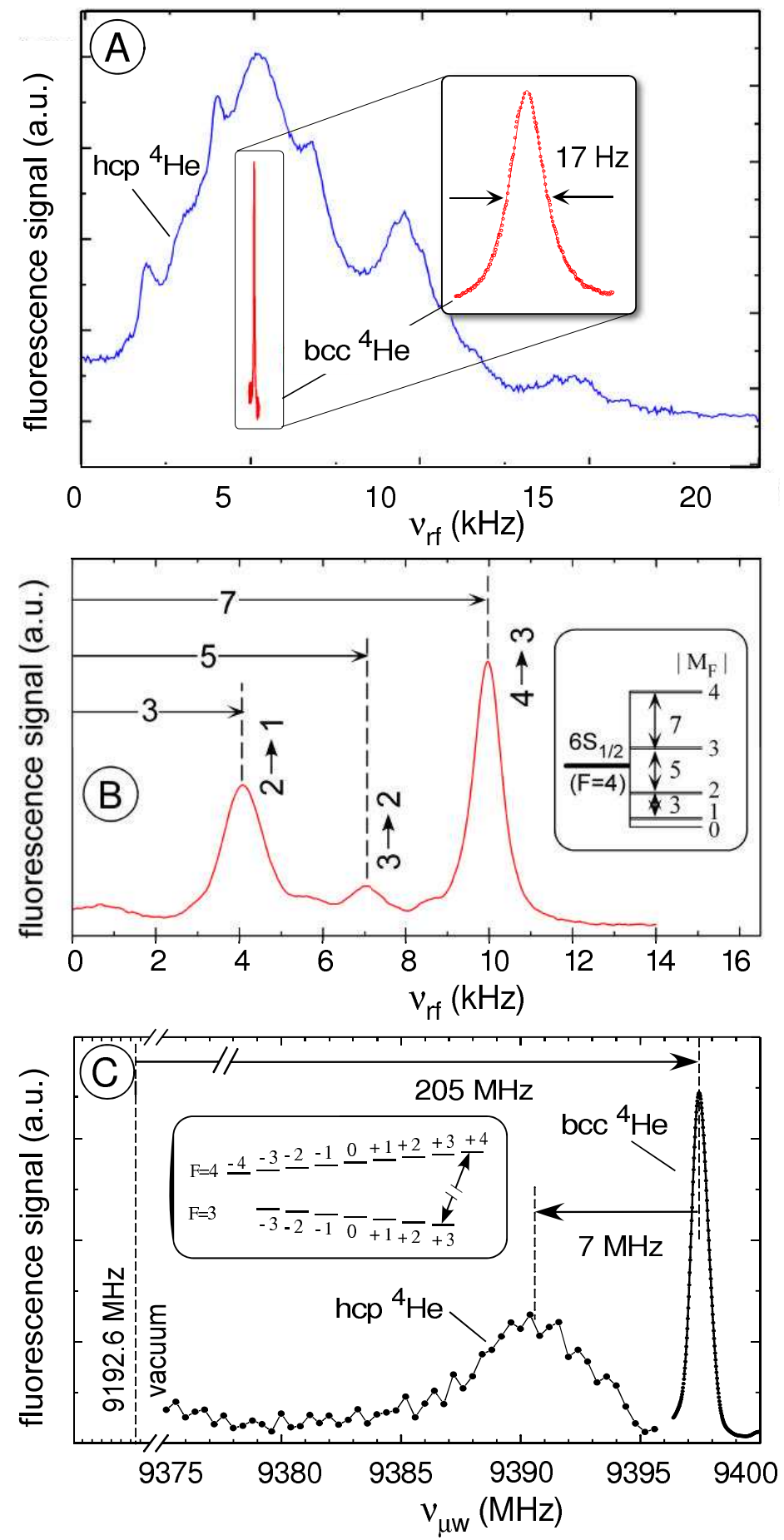

Fig. 33. Matrix effects in ground state magnetic resonance spectra. A) Comparison of intramultiplet (Zeeman) magnetic resonance spectrum of cesium in bcc and hcp ${ }^{4} \mathrm{He}$ in the same magnetic field of $\approx 1.4 \mu \mathrm{T}$. B) Zero field magnetic resonance spectrum in the $F=4$ ground state of cesium trapped in hcp ${ }^{4}$ He. The insert shows the level structure expected from a third order perturbation by quadrupolar bubble deformations. The lines are slightly rf power broadened and the weaker lines at the midpoints between the main lines represent $\Delta M=2$ two photon transitions. The magnetic resonance spectrum shown in $\mathrm{A}$ ) can be understood as the Zeeman splitting of this spectrum. C) Comparison of intermultiplet (hyperfine) magnetic resonance spectrum of cesium in bcc and hcp ${ }^{4} \mathrm{He}$. Both spectra in C) were recorded in a single scan in which the phase transition was induced by a pressure change during the scan between the two resonances. The shifts are discussed in the text. 


\subsubsection{Hyperfine transition}

The $S-D$ mixing coefficient inferred above from the zero field spectrum may also be estimated from the experimentally observed shift of the Cs clock transition frequency at the bcc-hcp phase transition. Fig. 33.C shows the $F=3 \rightarrow F=4$ hyperfine transition in Cs recorded in the bcc and in the hcp phases of solid He. In the bcc phase the resonance frequency shows a blue shift of approximately $200 \mathrm{MHz}(\approx 2 \%)$ with respect to the transition in the free atom. Since the hyperfine coupling constant representing the Fermi contact interaction is proportional to $\left|\Psi_{6 S}(r=0)\right|^{2}$, this shift can be explained by the matrix-induced compression of the valence electron's wavefunction onto the nucleus [73,48]. At the bcc-hcp phase transition the He density increases and one can estimate that this should imply a further blue shift by approximately $2 \mathrm{MHz}$. This is in contradiction to the experimental observation of a $7 \mathrm{MHz}$ shift to lower frequencies. Compared to the estimated pressure shift the line is thus redshifted by $9 \mathrm{MHz}$ by the bubble deformation. In [70] we have speculated that this shift is a consequence of a quadrupolar bubble deformation, and we have inferred the corresponding $S-D$ mixing coefficient to be on the order of $3 \%$, based on a normalization argument. An equivalent way to infer the mixing coefficient starts from the third order energy shift induced by the deformed bubble (Eq. 27). If in that equation we assume that only the lowest lying $5 D$ state contributes to the hyperfine shift we find a mixing coefficient of

$$
\varepsilon_{6 S, 5 D}=\sqrt{\frac{5 \Delta E_{h f}^{\text {def.bub. }\left(6 S_{1 / 2}\right)}}{A_{\mathrm{hf}}\left(6 S_{1 / 2}\right)}},
$$

which evaluates to $\varepsilon_{6 S, 5 D}=6.9 \%$ when using $A_{\mathrm{hf}}\left(6 S_{1 / 2}\right)=2348 \mathrm{MHz}$, the hyperfine coupling constant observed in bcc ${ }^{4} \mathrm{He}$. Note that the latter value for the mixing coefficient is compatible with the $3 \%$ value inferred by us earlier [70] since here we define $\varepsilon_{6 S, n D}$ in terms of a reduced matrix element $\left\langle n D\left\|V_{\text {bub }}^{\text {hcp }}\right\| 6 S\right\rangle$, while in [70] we used the normal matrix element $\left\langle n D\left|V_{\mathrm{bub}}^{\mathrm{hcp}}\right| 6 S\right\rangle$, the two definitions differing by $\sqrt{5}$.

\subsubsection{The $S$-D mixing matrix element}

In the previous paragraphs we have estimated the S-D-mixing coefficient $\varepsilon_{6 S, 5 D}$ by a simplified analysis which took only deformed bubble-induced perturbations by the lowest lying state into account and have obtained two values which are of the same order of magnitude, but which differ by a factor of 2.5. There are two possible reasons for this discrepancy. First, one may blame our simplified analysis which has considered only a single state, while all bound (and continuum) states contribute. Moreover, excited states contribute with different weighting factors to the hyperfine shift and the zero field splitting according to Eqs. 27 and 28, 29. A second reason for the discrepancy may be due to the following fact. Experiments measure the change of an atomic property (in the case of the hf shift) or the appearance of a novel property (zero field splitting) when passing the bcc-hcp phase boundary. Our theoretical model, on the other hand compares effects in bubbles with a quadrupolar deformation with those in a spherical bubble with the same He bulk density, and thus ignores any possible effect of the deformation that was already induced when passing from liquid (spherical bubble) to bcc (bubble with hexadecupole deformation). In section 9.8.2 we have argued that the passage from liquid to bcc gives no measurable zero-field line splitting, so 
that the effect seen on the bcc-hcp transition is equivalent to the liquid-hcp transition. This situation is different with respect to the hyperfine shift. A deformation-induced shift on the liquid-bcc transition has never been addressed experimentally.

In any case our analysis permits us to state that the $6 \mathrm{~S}-5 \mathrm{D}$ mixing coefficient lies somewhere between $3 \%$ and $7 \%$. From this we can obtain the following estimate for the $6 S-n D$ matrix element

$$
\left\langle 5 D\left\|V_{\mathrm{bub}}^{\mathrm{hcp}}\right\| 6 S\right\rangle=\varepsilon_{6 S, 5 D}[E(5 D)-E(6 S)] \approx 400 \ldots 1000 \mathrm{~cm}^{-1},
$$

where we have used that the energy of the $6 \mathrm{~S}-5 \mathrm{D}$ transition is $\approx 15000 \mathrm{~cm}^{-1}$ for Cs in solid He. We note that this matrix element is 2 to 5 times larger than the spin-orbit coupling constant $A_{L S}(5 D)$ in solid He and many orders of magnitude larger than the hyperfine coupling constants of the 5D state. In our theoretical third order perturbation calculation (section 4.3.1) we have assumed a perturbation operator consisting of the sum of the hyperfine interaction $H_{\mathrm{hfs}}$ and the deformed bubble operator $V_{\mathrm{bub}}^{\mathrm{hcp}}$. That operator was applied to spin-orbit eigenstates. In a strict sense one should perform a degenerate state perturbation treatment using the operator $V=V_{\mathrm{bub}}^{\mathrm{hcp}}+H_{\mathrm{hfs}}+V_{L S}$, where $V_{L S}$ is the spin-orbit operator. However, we have verified by an explicit calculation that a third order treatment with the operators $V_{\text {bub }}^{\text {hcp }}$ and $V_{L S}$ yields neither $F$ - nor $M$-dependent shifts of the ground state energy.

\subsubsection{Deformation-induced forbidden transitions}

Forbidden $\Delta M=2,3$ transitions have been observed in ODMR experiments in hcp solid He $[188,70]$ in an external magnetic field. This is another manifestation of the quadrupolar perturbation of Cs atoms in the hcp phase. As shown in Sec.4.3.1, the perturbation induces an admixture of $M_{F}=2,3$ states to the $M_{F}=4$ state (the only one populated by optical pumping), hence dipole magnetic transitions towards $M_{F}=1,2$ become allowed. A typical spectrum containing the allowed $\Delta M=1$ and the two forbidden $(\Delta M=2,3)$ lines is shown in Fig. 34. In those experiments the frequency of the oscillating magnetic field was kept fixed and the spectra were recorded by scanning the static magnetic field.

\subsubsection{Dynamic bubble deformation-induced effects}

Although the $T_{1}$ times of spin-polarized alkali atoms in condensed helium are quite long (1-2 seconds in the case of Cs), it is not clear at present why they are not much longer, since there is no direct coupling of the (electronic or nuclear) spins to the He atoms in a spherical bubble. In section 9.8.2 we have shown that the total atomic angular momentum has a weak coupling to the axis of a bubble with a quadrupolar deformation. Atomic bubbles are not static objects, but undergo zero-point shape oscillations that can be interpreted as collective motions resulting from a multipole decomposition of individual oscillations of the ensemble of He atoms forming the bubble interface. It is thus reasonable to assume that the finite $T_{1}$ times result from a depolarization induced by a coupling of the spin to dynamic quadrupolar (and higher order) oscillations. Since the bubble oscillation frequency is many orders of magnitude larger than the characteristic coupling frequency of the spin to the bubble axis one has the theory of motional narrowing [203] to infer the effective lifetime of the spin polarization. A quantitative analysis along those lines has not been performed so far. 


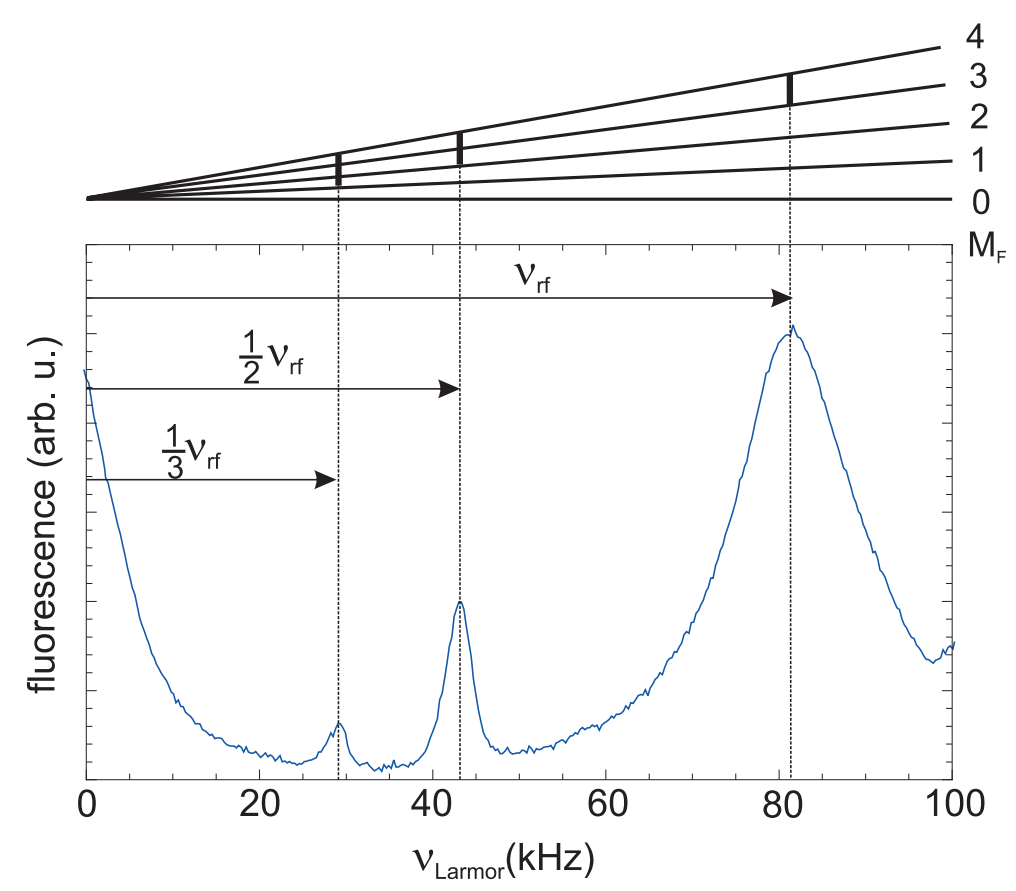

Fig. 34. Allowed $\Delta M_{F}=1$ and forbidden $\Delta M_{F}=2,3$ transitions in hcp phase in a strong rf field. The spectrum was recorded by scanning the static magnetic field, with the frequency of the oscillating magnetic field $\nu_{r f}$ fixed.

\section{Impurity-helium solids}

In this section we discuss two classes of impurity-helium systems in which a presence of atomic or molecular impurities affects properties of a helium matrix and results in a formation of a macroscopic solid objects bound by the interaction between the impurity particles and helium atoms.

\subsection{Solid helium stabilized by impurity ions}

\subsubsection{Positive ions in condensed helium}

In contrast to neutral alkali atoms, the positively charged alkali metal ions in condensed He reside in a very tight trapping sites in which the surrounding He density is significantly higher than in bulk He. Since the ions have closed shells there is no Pauli repulsion and the helium atoms can approach much closer to the alkali core than in the case of a neutral atom. Because of the absence of repulsive forces no bubble is formed. However, the He atoms are polarized and attracted by the strongly inhomogeneous electric field near the ion, an effect known as electrostriction. Already in 1959 it was suggested [204] that due to electrostriction positive $\mathrm{He}^{+}$ions in liquid He would attract and bind a number of neutral He atoms. The electrostrictive forces are so strong that they compress the nearest neighbor He atoms to form a solid crust around the ionic core to form a complex which has been given the name of snowball. The snowball model can explain the relatively low mobility of positive ions in liquid He that was observed in many experiments [34,35,205]. Mobilities of impurity ions in liquid He, including the positive ions of alkali and alkaline-earth elements were studied experimentally by $[206,207,114,115,208-210]$ and a generalization of Atkins' 
model was presented in [211,212]. For the alkali-metal ions both measurements [114] and the snowball model [211] yield snowball radii of about $8.4 \AA$, slightly larger than for the $\mathrm{He}^{+}$ ion.

In the case of the singly charged alkaline-earth ions, the remaining valence electron interacts repulsively with the surrounding He atoms. The authors of [211,212] conclude that those ions in liquid He form bubbles rather than snowballs, and the bubble radius was estimated to be $R_{b} \approx 4.7 \AA$, considerably smaller than that of alkali atoms. Still, electrostriction produces a layer with increased He density outside the bubble. The absorption lines of alkali ions lie in the deep UV part of the spectrum and have never been studied in condensed helium. Spectroscopic studies of $\mathrm{Ba}^{+}$ions were reported $[213,116]$, and the observed spectral line shifts and broadenings were rather small supporting the assumption of the bubble-type trapping site.

Numerical Monte Carlo simulations of the snowballs formed by impurity ions in liquid He were recently reported in [214-217]. They confirmed the presence of solid He structures surrounding the alkali ions. The He atoms are strongly localized, in particular for $\mathrm{Na}^{+}$and form two well-defined solid shells. The total number of bound He atoms ranges from 22 for $\mathrm{Na}^{+}$to 10 for $\mathrm{Cs}^{+}$. For the alkaline-earth ion $\mathrm{Mg}^{+}$also a snowball structure was obtained. The He atoms in that case are much less localized and the number of bound atoms is only 6.

Experimentally the effect of increased He pressure on the snowball structure was mainly investigated in mobility studies, and the mobility was found to decrease with He pressure. It was found that even at a He pressure approaching the solidification point, no dramatic increase of the snowball radius occurs $[34,35,218]$. The analysis of $[219,218]$ shows that the liquid-solid surface tension at the boundary of the snowball stabilizes its growth even at pressures slightly above the solidification point. In the absence of other nucleation centers this would result in a metastable supercooled liquid. Since in experiments the crystallization easily starts at the container walls, the authors of [219] conclude that it should be very difficult to observe crystal nucleation on positive ions, although the nucleation at an electrically charged metallic tip is a well known effect [220].

The motion of positive ions in solid $\mathrm{He}$ was studied only for $\mathrm{He}^{+}$ions produced via the ionization of He atoms by energetic electrons emitted by a radioactive $\beta$-emitting source [221-225]. In solid He, close to the melting point the mobility is several $(3-6)$ orders of magnitude lower than in liquid and decreases further with increasing pressure. The effect of crystal anisotropy on the mobility of positive ion was observed experimentally [224] on the melting curve of the hcp phase. It was found that the motion of $\mathrm{He}^{+}$along the $c$-axis of the crystal is about 200 times faster than in the orthogonal direction.

\subsubsection{Visual observations of "iceberg" structures}

Since our early studies of Cs-doped solid He we have observed an interesting phenomenon that takes place during the melting of the doped sample. The observations are illustrated in Fig. 35. As described in Sec. 6.1, the $\mathrm{Cs}(\mathrm{Rb})$-doped part of the solid He matrix has the shape of a column, approximately $3 \mathrm{~mm}$ in diameter and up to $2 \mathrm{~cm}$ in height. It can be distinguished from the surrounding transparent pure solid helium by its grey(blue)color (Fig. (a)), which is due to $\operatorname{Cs}(\mathrm{Rb})$ clusters [127]. When the helium pressure is released below 26.6 

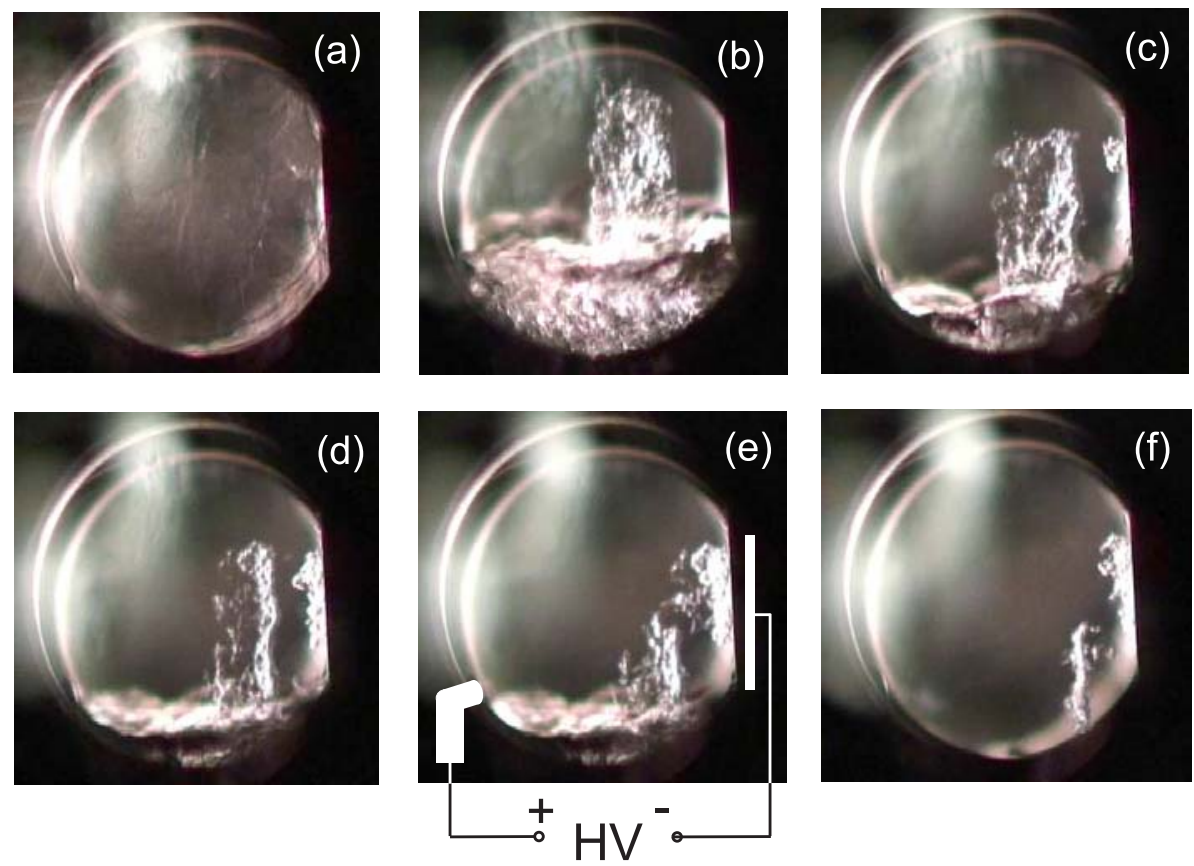

Fig. 35. Photographs taken trough a cryostat window (diameter $2 \mathrm{~cm}$ ) during the melting of a Cs-doped He crystal with applied electric field. (a) - before melting; (b) - liquid-solid interface moves down, in front of the window; (c) - "iceberg" in liquid He; (d)-(f) fragments of the "iceberg" are attracted to the negative electrode.

bar (at $1.5 \mathrm{~K}$ ), the crystal starts to melt, and the melting proceeds from top to bottom of the pressure cell, as shown in Fig. 35(b). During the melting the doped part of the crystal remains solid and keeps its column-like shape. Finally, as shown in Fig. 35(c), the doped column is surrounded by liquid He only, a structure that we refer to as iceberg. Under constant temperature and He pressure the iceberg survives for as long as several hours, gradually loosing its mechanical strength and finally breaking up into several fragments that float to the bottom of the cell, escaping observation.

The lower row of photographs in Fig. 35 shows the evolution of the iceberg in external electric field created between two electrodes mounted inside the pressure cell (shown in Fig. 35(e)) that produce a field strength of about $10 \mathrm{kV} / \mathrm{cm}$ at the location of the iceberg. As one can see in Fig. 35(d)-(f), the fragments of the iceberg in the electric field, rather than falling down, move towards the electrodes and stick on the latter. We could observe fragments sticking to the positively or to the negatively charged electrode, suggesting that charges of both signs with a nonuniform spatial distribution are present in the iceberg.

\subsubsection{Interferometric study}

Recently we reported a first quantitative study of the iceberg [127], using an interferometric technique (described in Sec. 6.2) to measure the index of refraction of the doped solid He column surrounded either by pure solid or liquid helium. The corresponding interferograms are shown in Fig. 36(a)-(c). In solid He the interferogram (Fig. 36(a)) displays no structure that can be associated with the doped part of the sample. From this observation we conclude that the additional refraction introduced by the dopants is negligible. On the other hand, the interferograms obtained when the iceberg is surrounded by liquid He clearly exhibit 

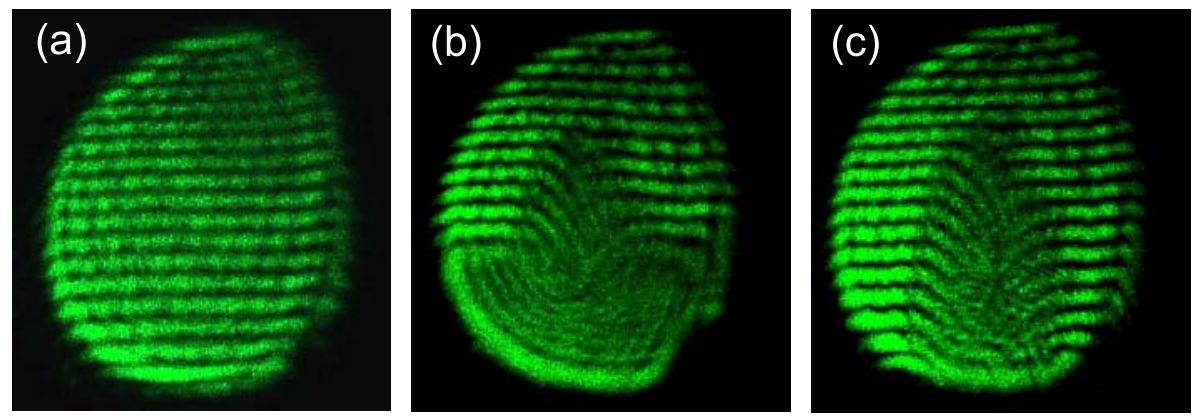

Fig. 36. Interferograms taken during the melting of a Cs-doped He crystal. (a) - before melting; (b) - liquid-solid interface moves down, in front of the window; (c) - "iceberg" in liquid He.

such a structure (Fig. 36(b) and (c)). Within the iceberg the interference fringes shift in the direction of increasing refraction index. The magnitude of the shift is proportional to the thickness of the iceberg and reaches a maximum on its axis, which has allowed us to infer the difference of the refractive indices between the iceberg proper and pure liquid He. Since the extra refraction is not due to the impurities, we conclude that it is a consequence of the increased density of the iceberg which is $\approx 3 \%$ larger than that of liquid He, while being below the density of pure solid He which, at the solidification point at $1.5 \mathrm{~K}$ is $7 \%$ larger than that of liquid He under the same conditions.

Our interpretation of these results takes into account the fact that the number density of neutral impurity particles, including atoms, dimers, and clusters is does not exceed $10^{10}$ $\mathrm{cm}^{-3}$. Moreover, since all neutral impurities strongly repel He atoms due to the Pauli principle the binding of He atoms in the iceberg structure is most likely due to positively charged alkali ions, which attract He atoms by electrostriction. The charges are probably produced during the laser ablation from the bulk metal target, a process which is known to produce large amounts of atomic and molecular ions. Those charged impurities are also responsible for the attraction of the iceberg fragments by charged electrodes.

\subsection{Impurity-helium solids bound by van der Waals forces}

\subsubsection{Structure}

A method for isolating atomic and molecular impurities in superfluid He has been suggested by E. B. Gordon and coworkers in 1974 [128]. In their experiments a gaseous jet of helium doped with impurity atoms/molecules was directed onto the surface of a superfluid He bath, where it was found to condense into a highly porous aggregation of solid impurity clusters surrounded by a shell of He, a structure which the authors refer to as impurity-helium solid. This type of implantation technique is discussed in detail in [226,227]. It was successfully applied to the rare gas atoms $\mathrm{Ne}, \mathrm{Ar}, \mathrm{Kr}$, and $\mathrm{Xe}$, as well as to nitrogen, hydrogen and deuterium. With the three latter species the dominant part of the dopants is implanted in molecular form, although a significant density of impurity atoms was also detected.

All those dopants posses a relatively compact closed-shell electronic configuration and the van der Waals attraction between the impurity particle and surrounding He atoms dominates their interaction, while the Pauli repulsion acts only at very small interatomic 
separations. A theoretical model developed in [129,228,226,13] assumes that each impurity atom or molecule is surrounded by a shell of 12 closely packed He atoms - a configuration equivalent to a substitution site in a He crystal - and shows that such a structure is stable under the experimental conditions. However, later experimental studies of the impurityhelium solid structure using X-ray diffraction, ultrasound propagation and electron-spin resonance (ESR) techniques, have shown that the typical impurity-helium solid consists of rather large impurity clusters. The cluster formation mechanism was discussed in [229,131], with the conclusion that the clusters are already formed in the gas jet before hitting the liquid He surface. A solid shell of He is then formed around each cluster due to the van der Waals attraction and prevents their further coalescence into larger particles. During the solvation process these impurity-helium clusters stick together and form the porous structure, whose properties have been extensively studied.

The impurity-helium solid samples decompose when heated, with a melting temperature that depends on the sample composition and that is larger than the evaporation temperature of pure liquid helium $(4.2 \mathrm{~K})$. The impurity-helium solid survives the complete evaporation of liquid He. However, the structure of the solid is affected by the warming process: small impurity clusters merge to form larger ones.

Recently, the implantation technique was modified in order to implant impurities into solid He $[130,131]$. Rather than condensing a gas jet into liquid He, the impurity-helium mixture is pressurized and brought into contact with the upper surface of a He crystal. The mixture solidifies while the pure He crystal is slowly pushed out of the pressure cell through a capillary at its bottom, until all of the initial helium is replaced by the doped solid. This method was applied to dope solid $\mathrm{He}$ with $\mathrm{N}_{2}$ and $\mathrm{O}_{2}$ molecules [131]. From the composition of the gas mixture the density in the solid is estimated to be $\approx 3 \times 10^{19} \mathrm{~cm}^{-3}$. The method was later applied to dope deuterium into solid He and to study isolated deuterium clusters [230].

Impurity-helium samples have been extensively investigated for different dopants (listed in Table 3) using optical spectroscopy, X-ray scattering, ultrasound propagation and electronspin resonance (ESR) methods. Below we give a brief description of the obtained results which were recently reviewed in [10-12] and which are complementary to our own studies of alkali-doped solid He.

\subsubsection{Optical spectroscopy}

A systematic spectroscopic study was reported for neon and nitrogen-helium solids [231,245]. The gas jet used for the immersion of the impurities was derived from a gas discharge of the He-impurity gas mixture, that produced a number of excited atoms and molecules. The most striking feature of this experiment is an extremely long-lived green luminescence emitted by the metastable ${ }^{2} D$ state of nitrogen atoms that could be observed for hours following the implantation. In addition to this, molecular bands of $\mathrm{N}_{2}$ dimers were observed. The luminescence spectrum taken during the thermal destruction of the sample also contains bands of the NO molecule and of atomic oxygen, due to an oxygen contamination of the He gas used for the deposition [245]. The Ne-He solid was investigated [231] by studying laser-induced fluorescence at electronic transitions of Ne atoms starting from the metastable lower state that is populated in the electric gas discharge before deposition. 


\begin{tabular}{|c|c|c|}
\hline dopant & reference & remark \\
\hline $\mathrm{Ne}$ & {$[231]$} & optical spectroscopy \\
& {$[232-234]$} & X-ray diffraction \\
& {$[233,235,236]$} & ultrasound propagation \\
\hline $\mathrm{Ar}$ & {$[226]$} & \\
$\mathrm{Kr}$ & {$[232,233,237]$} & X-ray diffraction \\
& {$[233,235,236]$} & ultrasound propagation \\
\hline $\mathrm{Xe}$ & {$[226]$} & diffraction \\
\hline $\mathrm{H}_{2}$ & {$[238-241]$} & ESR \\
\hline $\mathrm{D}_{2}$ & {$[233,234,242,243]$} & X-ray diffraction \\
& {$[233,235,236]$} & ultrasound propagation \\
& {$[238-243]$} & ESR \\
\hline $\mathrm{N}_{2}$ & {$[128,231,229,244,245]$} & optical spectroscopy \\
{$[232-234]$} & {$[233,235,236]$} & X-ray diffraction \\
& {$[246-248]$} & optical spectroscopy \\
\hline
\end{tabular}

Table 3

Atomic and molecular gases studied in experiments on impurity-helium solids.

Luminescence spectra from metastable nitrogen and oxygen atoms, as well as from molecular nitrogen from samples produced by the analogous technique were also reported by a group at Jyväskylä University [229,244]. The authors compared the spectra of $\mathrm{N}$ and $\mathrm{N}_{2}$ observed in nitrogen-helium solids to those in a pure solid nitrogen matrix and find them to be very similar, thus supporting the conclusion that the emitting particles in the nitrogenhelium solid are in fact surrounded by solid nitrogen and not by helium, i.e., that they are embedded in nitrogen clusters.

\subsubsection{X-ray diffraction}

The structure of impurity-helium solids formed by $\mathrm{Ne}, \mathrm{Kr}, \mathrm{N}_{2}$, and $\mathrm{D}_{2}$ impurities, as well as by a $\mathrm{D}_{2}$-Ne mixture in liquid He was investigated in a series of X-ray diffraction experiments [232-234,237,242,243]. The observed diffraction patterns consist of overlapping peaks that are characteristic for liquid He and for impurity crystals with a fcc structure. The average density of impurity atoms or molecules, estimated from the ratio of He and impurity peak intensities in the scattering diagram, was on the order of $10^{20} \mathrm{~cm}^{-3}$. The scattering pattern due to the impurity is strongly broadened suggesting a small size of the scattering particles. The analysis of the peak widths has allowed the authors to derive a characteristic size of approximately $5.9 \mathrm{~nm}$ for neon impurity clusters [232,237], $6.2 \mathrm{~nm}$ for argon [237], 5.4 $\mathrm{nm}$ for krypton [237], $5 \pm 2 \mathrm{~nm}$ for nitrogen [233], and between 7.5 and $13 \mathrm{~nm}$ for deuterium $[242,243]$. Defects of the crystalline structure of the clusters also affect the scattering pattern by increasing the peak widths and by lowering the intensities of higher order peaks. The authors of $[232,233]$ have demonstrated that the inclusion of crystalline defects significantly improves the agreement between the experimental and calculated scattering patterns. In 
a more recent study [237] an even better agreement between experimental and modeled scattering data was obtained for $\mathrm{Ne}$, Ar, and $\mathrm{Kr}$ clusters in liquid $\mathrm{He}$, assuming that the clusters possess either an icosahedral or a decahedral structure.

Warming of the sample results in an increase of the characteristic cluster size due to the coalescence of small clusters into larger ones. The peaks in the corresponding scattering pattern become narrower due to the larger size of crystals and to annealing of the defects. Finally, the scattering pattern becomes identical to that of the bulk impurity crystal, indicating a collapse of the impurity-helium solid.

\subsubsection{Ultrasound propagation}

The velocity of ultrasound $(5 \mathrm{MHz})$ propagation and its attenuation in impurity-helium solids formed by $\mathrm{Ne}, \mathrm{Kr}, \mathrm{D}_{2}$ and $\mathrm{N}_{2}$ in liquid $\mathrm{He}$ was measured in $[233,235,236]$. It was shown that the properties of the deuterium-helium solid are identical to those of pure liquid He due to the closeness of the molecular mass of $\mathrm{D}_{2}$ and He. On the other hand, heavier impurities significantly increase the attenuation which is further increased by a compression of the sample. The ultrasound velocity is much less affected, and decreases only slightly due to the larger density of the sample.

The attenuation of the sound wave results from the friction of the layers of normal fluid. By analyzing the dependence of the ultrasound attenuation on the sample temperature the authors of $[236,233]$ obtained information on the characteristic pore size of the impurityhelium structure. Their method is based on the fact that the sound wave can penetrate only into pores whose diameter is larger than the viscous penetration depth

$$
\delta_{\text {visc }}=\sqrt{\frac{2 \eta}{\omega_{\text {sound }} \rho_{n}}},
$$

which depends on the viscosity of He $\eta$, the ultrasound frequency $\omega_{\text {sound }}$ and the density $\rho_{n}$ of the normal component of superfluid He. Therefore the normal fluid inside the pores with a diameter below $\delta_{v i s c}$ is decoupled from the motion and does not contribute to the attenuation. The normal fluid density increases and the corresponding viscous penetration depth decreases as the sample is heated. There exists a temperature range, where the attenuation strongly increases with temperature because an increasing number of pores start to contribute. The lower boundary of this range gives the largest pore diameter. For the Ne-, $\mathrm{Kr}-$, and $\mathrm{N}_{2}$-He solids pore diameters of 320,530 , and $860 \mathrm{~nm}$, respectively were inferred[236,233].

\subsubsection{ESR studies}

When molecular gases $\left(\mathrm{H}_{2}, \mathrm{D}_{2}, \mathrm{~N}_{2}\right)$ are condensed in liquid helium after passing through a gas discharge, they form clusters that also contain large amounts of the corresponding impurity atoms. Their electron-spin resonance (ESR) spectra were studied in [246,238$243,247,248]$. ESR spectra were observed using a standard ESR spectrometer operating at 9 GHz. The spectrum is scanned by varying the static magnetic field in the range of $0.28-0.36$ T. At those high fields the electron spin $\mathbf{S}$ and nuclear spin $\mathbf{I}$ in the atom are decoupled and the selection rules $\Delta M_{S}= \pm 1, \Delta M_{I}=0$ hold for dipole magnetic transitions. The ESR spectrum thus consists of $2 \mathrm{I}+1$ components, i.e., one observes doublet structures in hydrogen and triplets in deuterium and nitrogen. By comparing the signal amplitude to the 
one from a ruby crystal with a known spin density, the absolute average number density of atoms in the impurity-helium sample could be determined. Atomic densities as high as $1.5 \times 10^{18}, 8 \times 10^{17}$, and $1.4 \times 10^{19} \mathrm{~cm}^{-3}$ were obtained for deuterium [243,242], hydrogen [238] and nitrogen [247], respectively. The local density of atoms within the cluster is significantly higher. It could be estimated from the spectral width of the resonances assuming that the broadening is due to spin-spin interactions. For $\mathrm{N}_{2}$-He samples a local atomic density of $8 \times 10^{20} \mathrm{~cm}^{-3}$ was obtained which corresponds to a $10 \%$ relative concentration of $\mathrm{N}$ atoms with respect to $\mathrm{N}_{2}$ molecules. The time dependence (on a time-scale of several hours) of the relative densities of $\mathrm{D}$ and $\mathrm{H}$ atoms in a mixed hydrogen/deuterium-helium sample was used to study the quantum tunneling reactions $\mathrm{D}+\mathrm{HD} \rightarrow \mathrm{D}_{2}+\mathrm{H}$ and $\mathrm{D}+\mathrm{H}_{2} \rightarrow \mathrm{HD}+\mathrm{H}$.

In addition to the main ESR peaks much weaker satellite lines were observed in both hydrogen and hydrogen/deuterium-doped samples [238,240]. These satellites were attributed to forbidden transitions involving a simultaneous flip of the electron spin in the atom and the proton spin in one of the neighboring $\mathrm{H}_{2}$ or $\mathrm{HD}$ molecules thus supporting the conclusion that the atoms are isolated inside clusters. The satellites due to the electron spin interaction with the deuteron spin could not be resolved due to the smaller magnetic moment of the deuteron. The ratio of intensities of the satellite and main ESR peaks provided information on the characteristic distance between the atom and the neighboring molecule and show that this distance is approximately $2 \mathrm{~nm}$, which corresponds to atoms trapped in interstitial sites in solid molecular hydrogen.

Electron spin echo measurements in hydrogen and deuterium were reported recently [12]. The modulation of echo signals due to the interaction the electron spin with both protons and deuterons was resolved and the typical number of $\mathrm{HD}$ and $\mathrm{D}_{2}$ molecules surrounding one deuterium atom was obtained.

A detailed analysis of the ESR lineshapes was reported [243] for deuterium-helium solids of different compositions, including solids with an admixture of neon. It was shown that the electron gyromagnetic ratio is practically not affected by the matrix, whereas the zerofield hyperfine splitting is reduced by approximately $1 \mathrm{MHz}$ with respect to the free atomic value of $327.4 \mathrm{MHz}$. Both results closely reproduce those obtained in solid $\mathrm{D}_{2}$ matrices. The longitudinal $T_{1}$ and transverse $T_{2}$ spin lifetimes were obtained from an analysis of the saturation of the ESR transition by the high power radio frequency field. The value of $T_{1}$ is determined by spin-lattice relaxation and is equal to $3 \mathrm{~ms}$, whereas $T_{2}=44 \mathrm{~ns}$, is limited by spin-spin relaxation.

\section{Other rare-gas matrices}

In this section we compare the atomic bubbles in solid He with the trapping sites of metal atoms isolated in solid Ne, Ar, Kr, and Xe matrices. The latter have been investigated since 1960ies by means of optical and ESR spectroscopy. A review of these studies one can find in $[162,9]$. Here we consider mostly alkali and noble metals as impurities since their spectra can be directly compared to those observed in condensed helium. 
Table 4

\begin{tabular}{|c|c|c|c|c|c|}
\hline element & $M$ & $\varepsilon(\mathrm{K})$ & $r_{m}(\AA)$ & $a(\AA)$ & $\alpha\left(\AA^{3}\right)$ \\
\hline $\mathrm{He}$ & 4 & 3.8 & 2.97 & 3.68 & 0.21 \\
$\mathrm{Ne}$ & 20 & 42.2 & 3.08 & 3.21 & 0.40 \\
$\mathrm{Ar}$ & 40 & 143.2 & 3.75 & 3.83 & 1.63 \\
$\mathrm{Kr}$ & 84 & 199.9 & 4.01 & 4.05 & 2.47 \\
$\mathrm{Xe}$ & 131 & 282.3 & 4.36 & 4.41 & 4.02 \\
\hline
\end{tabular}

Some parameters of rare gas atoms: atomic mass $M$, depth $\varepsilon$ and position $r_{m}$ of the potential well of the interatomic pair potential [249], next neighbor distance in the crystal $a$ and electric polarizability $\alpha$ from [9]. For He $\varepsilon, a$, and $\alpha$ are taken from $[15],[16]$, and [250], respectively.

\subsection{Trapping site structure}

In contrast to He, other rare gases solidify without external pressure and form face-centered cubic (fcc) crystalline structures. Due to the stronger interatomic interactions (see Table 4) and larger atomic masses these matrices are more rigid and have negligible zero point fluctuations, so the atoms in such crystals are well localized. As one can see in Table 4, the characteristic next-neighbor distance $a$ in those crystals is determined mostly by the position $r_{m}$ of the minimum in the corresponding interatomic pair potential. Except $\mathrm{Ne}, a$ in rare gas solids is larger than in solid He and increases when going from Ne to Xe. Due to the larger polarizability (see Table 4) of the heavier rare gases they have a stronger attractive interaction with alkali atoms at intermediate interatomic distances which results in more compact trapping sites. It is usually assumed that in the heavier rare gas matrices ( $\mathrm{Kr}$ and $\mathrm{Xe}$ ) most alkali atoms occupy substitution sites, whereas for Ne and Ar trapping sites composed of four adjacent vacancies was suggested. In most experiments a number of spectral features is observed that can be attributed to different trapping sites within the same sample. Their relative contribution strongly depends on the preparation procedure and can be modified by annealing the sample.

For the theoretical modeling of the trapping site structure two approaches were proposed in the 1980ies, both in close analogy with those used for atomic bubbles in He (discussed in Sec. 3.2). The alkali-matrix interaction is calculated using either a pseudopotential method [252], or using pair potentials [253]. In both approaches the rare gas atoms are placed at fixed positions in a discrete lattice structure in contrast to the uniform density distribution assumed for helium (see Sec. 3.2). Monte Carlo studies were reported for Na atoms in solid Ar [254] and for Li in solid Ne [255], which yielded both single substitution sites and four vacancy sites. More recently, substitution sites with several adjacent vacancies were studied theoretically in [256] for $\mathrm{Li}$ and $\mathrm{Na}$ in $\mathrm{Ne}, \mathrm{Ar}, \mathrm{Kr}$, and $\mathrm{Xe}$.

\subsection{Optical absorption}

The $n S \rightarrow n P$ transitions of matrix-isolated alkali atoms have been extensively studied by absorption spectroscopy in Ar, Kr, and Xe matrices [257-272] (for a review see [9]). The analysis of those spectra is complicated by the presence of several different trapping sites in the sample. However, a common feature of all spectra is a triplet structure that replaces the fine-structure doublet of the $n S \rightarrow n P$ transition in free alkali atoms. Depending on the tightness of the trapping site, the absorption lines are more or less blue shifted and are com- 


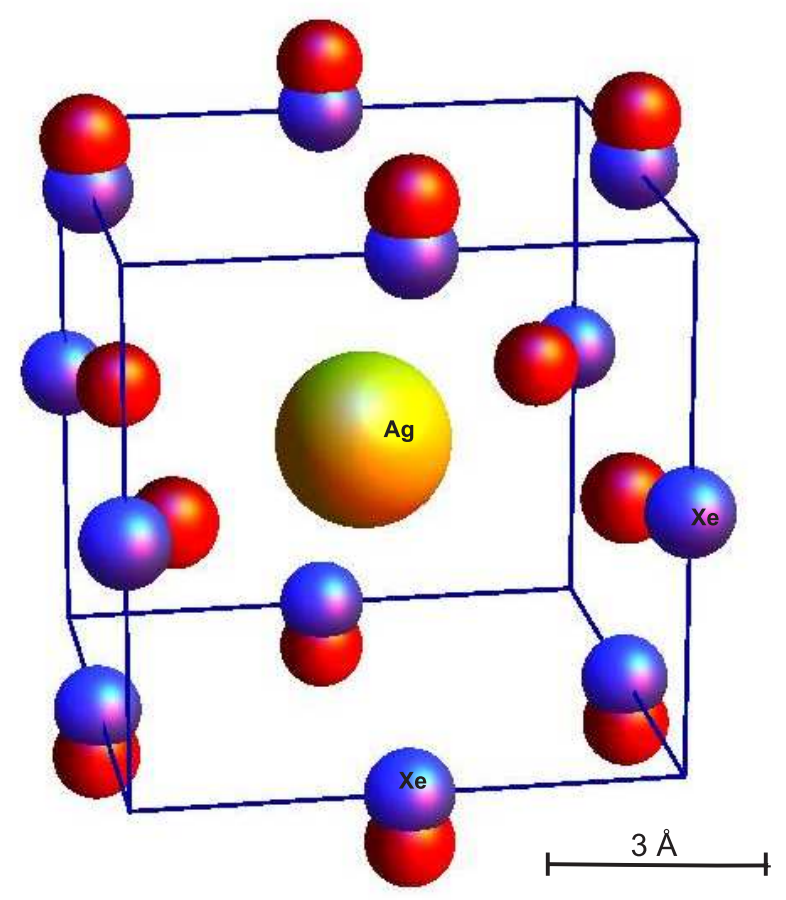

Fig. 37. A silver atom (in yellow) surrounded by an fcc cage of 12 Xe atoms (in blue). The Xe atoms (in red) are displaced by a relaxation of the cage following the laser excitation of the silver atom to the $P_{3 / 2}$ state. Quantitative values for the displacements of Xe atoms are taken from [251].

monly classified as "red", "blue", and "violet" triplets. The separations between neighboring triplet components for all alkalis and rare gases lie in the range of $100-500 \mathrm{~cm}^{-1}$. For Cs and $\mathrm{Rb}$ they are comparable to the fine-structure splitting in the free atom, whereas for the light alkalis the fine-structure splitting is much smaller than the triplet splittings. Due to their large spectral widths, the individual components of the triplet structures strongly overlap and peak positions reported in different publications vary considerably. Triplet structures of the $n S \rightarrow n P$ transitions have also been observed in absorption spectra of matrix-isolated noble metal atoms [273-278]. In those systems usually a single (substitution) trapping site is observed.

The use of magnetic circular dichroism (MCD) measurements yields a significant improvement in the spectral resolution with respect to absorption spectroscopy. In MCD experiments a strong external magnetic field lifts the Zeeman degeneracy of the magnetic sublevels. The corresponding splitting is however orders of magnitude smaller than the optical linewidth, so that it cannot be resolved in a normal absorption spectrum. Since left and right circularly polarized light is absorbed only by $\Delta M=-1$ and $\Delta M=+1$ components respectively, which each have Zeeman shifts of opposite signs, a differential absorption spectrum for the two polarizations yields a dispersively shaped resonance. Due to the high sensitivity of polarization measurements the dichroism can be measured very precisely and helps to separate overlapping spectral lines. MCD spectra were reported for alkali atoms [266,267] and noble metal atoms [279,276-278] in different rare-gas matrices. A numerical modeling of MCD spectra was recently reported [280,281]. 
The triplet structure is a consequence of the splitting of the excited $n P$ state induced by the interaction with the rare gas atoms via two different effects. First, the spin-orbit interaction in the impurity atom is modified by the so-called heavy-atom effect [282,283], which is particularly pronounced for light alkali atoms. The effect is interpreted as an admixture of excited orbitals of the rare-gas atoms to the orbital of the impurity valence electron, that arises from the overlap of their wavefunctions in a tight substitution site. The spin-orbit operator of the whole defect is given by

$$
H_{S O}=\Delta_{M} \mathbf{L}_{M} \cdot \mathbf{S}+\Delta_{R G} \sum_{k=1}^{N} \mathbf{L}_{k} \cdot \mathbf{S},
$$

where $\Delta_{M}$ and $\Delta_{R G}$ are spin-orbit coupling constants of the impurity and rare-gas atoms respectively and where the sum is taken over all rare-gas atoms forming a trapping site $(N$ $=12$ for a substitution site in an fcc crystal). The spin-orbit coupling in heavier rare gases is much more pronounced for light alkali atoms. When the electronic wavefunction contains an admixture of rare-gas atomic orbitals, the second term of Eq. 44 produces a contribution to the fine-structure splitting that can be significantly larger than that of the first term. In fact, for $\mathrm{Li}$ and $\mathrm{Na}$ in Xe or $\mathrm{Kr}$ matrices the observed fine-structure splitting is completely determined by the spin-orbit interaction of the rare gas.

The spin-orbit coupling by itself splits the $n P$ state into two components: a $n P_{1 / 2}$ orbital and a doubly degenerate $n P_{3 / 2}$ orbital. The triplet structure is a consequence of the lifting of the degeneracy in the $n P_{3 / 2}$ state by the interaction with the rare gas atoms. In the early work it has already been pointed out [257], that this occurs only when the multipole expansion of the interaction potential contains a term of quadrupolar symmetry. In other words, the trapping site structure should posses a preferred direction, for example a site formed by two adjacent vacancies, as suggested in $[252,253]$. However this possibility was ruled out by later studies using magnetic circular dichroism (MCD) and electron spin resonance (ESR) techniques [266,267,284]. It was shown that the trapping site in heavier rare gases is a substitution site in the fcc crystal and therefore possesses a cubic symmetry. As discussed in Sec. 4.3 the angular dependence of the cubic lattice Hamiltonian contains spherical harmonics with $l=0,4,6,8, \ldots, m=0, \pm 4, \pm 8, \ldots$ and therefore does not split $n S$ and $n P$ states. The splitting of the $n P_{3 / 2}$ state was therefore attributed to dynamical distortions (oscillations) of the cage formed by the rare gas atoms surrounding the impurity atom. The phenomenon is well known in matrix-isolation spectroscopy as Jahn-Teller effect. As discussed in $[282,276,267]$ the main contribution comes from tetragonal stretching and bending $\left(e_{g}\right)$ modes, and a smaller contribution from trigonal $\left(t_{2 g}\right)$ modes. Calculations of Jahn-Teller spectra were presented for K in solid Ar [265], for Li in Ar, Kr, and Xe [266,285], and for $\mathrm{Na}$ in $\mathrm{Xe}$ [267]. We also mention related work on the $n S \rightarrow n P$ transitions of Ag and $\mathrm{Au}$ in rare gas matrices [251]. The measured and calculated absorption spectra of Ag-doped solid Xe [251] is reproduced in Fig. 38. The two components of the $D_{2}$ absorption line are labeled "+JT" and "-JT", and the $D_{1}$ line is labeled "SO". The fourth component in the experimental spectrum appears due to the degeneracy of the perturbed $4 P$ level of the silver atom and the $6 S$ level of xenon.

The Jahn-Teller coupling parameters are usually obtained from fits or moment analysis of experimental absorption and MCD spectra for which the fitted lineshapes yield a reasonable 
agreement with experimental results.

The absorption spectra of Cs in solid He discussed in Sec. 7.3 also possess a triplet structure. The analysis is greatly facilitated by the fact that all atoms are trapped in identical trapping sites. The spin-orbit interaction in Cs atom is the strongest of all alkalis with a fine structure splitting of $554 \mathrm{~cm}^{-1}$, and the perturbation by the He matrix is much smaller than by other rare gas matrices. Immediately after the excitation, when the excited Cs atom interacts with the small He bubble, there is a large overlap of the Cs atom's $6 P$ orbital and the electronic density of the He atoms that form the bubble. However, the overlap integral of the corresponding wavefunctions vanishes since a large number of strongly delocalized He atoms is involved. Therefore no modification of the Cs spin-orbit coupling is expected.

The splitting of the $6 P$ state in condensed He is still dominated by the spin-orbit interaction in the Cs atom, while the interaction with the matrix results in an increase of that splitting by approximately $100 \mathrm{~cm}^{-1}$ and in the appearance of a secondary doublet structure in the blue component (the $D_{2}$ line). In liquid He the alkali atom is trapped in a spherical bubble (see Sec. 3.2) whose symmetry is even higher than that of the substitution site in the fcc crystal. As pointed out in $[44,46]$ the double structure of the $D_{2}$ absorption line in this case is due to the Jahn-Teller effect arising as a consequence of bubble shape oscillations. Two quadrupole oscillation modes were considered, proportional to $Y_{0}^{(2)}$ and $Y_{2}^{(2)}+Y_{-2}^{(2)}$, respectively. The former is a close analogue of the $e_{g}$ stretching mode of the cubic cage, in which the eight out-of-plane atoms move away from the impurity atom whereas the four atoms in the equatorial plane approach the impurity, and vice-versa. The calculations of the spectral contours of the $D_{2}$ absorption lines of $\mathrm{Cs}$ and $\mathrm{Rb}$ in liquid He $[44,46]$ included no fitting parameters. The calculated line splitting underestimates the observed one by a factor of 0.7. This discrepancy can be attributed either to higher order vibration modes not included in the model, or to a too small set of basis functions used to describe the perturbed electron wavefunction.

According to our argumentation in Sec. 4.2, the trapping site in an hcp He matrix represents a basically spherical atomic bubble with a small static quadrupolar deformation. The Cs-bubble interaction potential possesses an angular dependence that lifts the degeneracy of the $6 P_{3 / 2}$ sublevels even without vibrations. The analysis of Sec. 4.3 shows that the interaction energy is comparable to that of the spin-orbit coupling and the triplet structure results from the interplay of those two effects. The situation is equivalent to that in the low symmetry trapping sites discussed in the early work on heavier rare gas matrices $[252,253]$.

In bcc solid He the static deformation of the spherical atomic bubble has the same symmetry as the substitution site in the heavier rare gas matrices. Only the dynamic Jahn-Teller effect can produce a splitting in the $D_{2}$ absorption line. This would provide essential information about bubble shape oscillations in solid He. Unfortunately, only the $D_{1}$ absorption lineshape has been studied experimentally up to date [48].

\subsection{Fluorescence}

Fluorescence spectra of alkali atoms isolated in rare-gas matrices were reported in [261264]. Typical spectra consist of two components that are strongly redshifted and that show a stronger broadening than the absorption lines. Similar results were obtained in experiments 


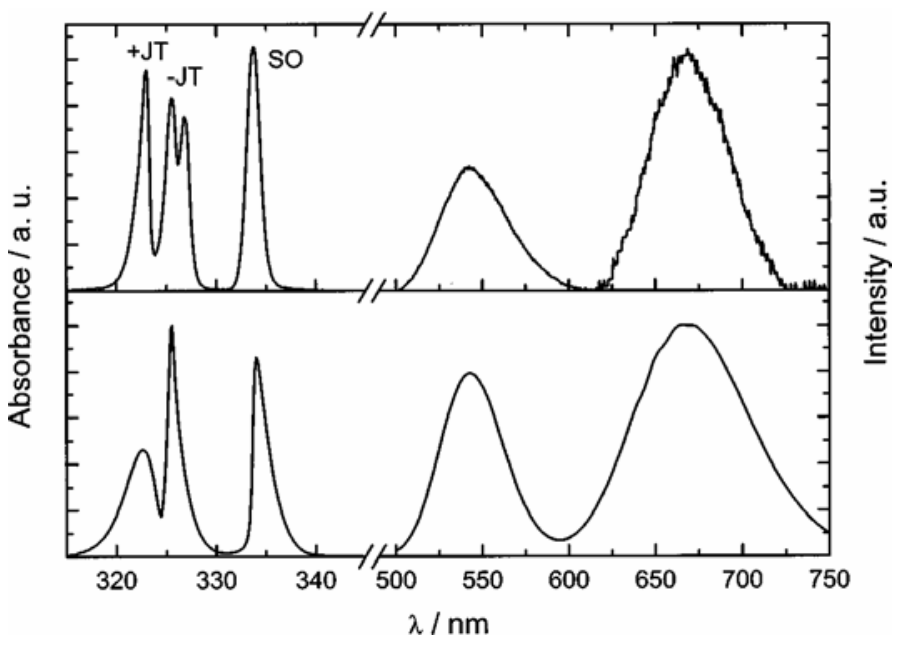

Fig. 38. Absorption (left) and fluorescence (right) spectra of Ag-doped solid Xe, taken from [251]. Upper panel - experiment; lower panel - theory. Reprinted with permission from [251]. Copyright 1999, American Institute of Physics.

with silver atoms as dopants [286,273,287,275], for which a quantitative interpretation was presented in [251] (Fig. 38). The redshift results from a shape relaxation of the trapping site following the excitation of the impurity atom. The cubic cage of the substitution site is deformed in order to better accommodate the excited orbital of the dopant. The energy of the excited state is lowered and at the same time the ground state energy strongly increases thus reducing the transition energy.

The model of [251] assumes that the deformation proceeds along the same coordinates that are responsible for the Jahn-Teller splitting of the excited $n P$ state observed in absorption. The new equilibrium configurations (for each $M_{J}$ component of the excited state) are found as minima in the total defect energy, using the interatomic pair potentials and assuming that only the rare-gas atoms in the first solvation shell are displaced. As discussed in Sec. 3.3 and 7.3, we use essentially the same approach in our treatment of the fluorescence spectra of alkali atoms in condensed $\mathrm{He}$, the difference being that the interaction between the first and the second solvation shells in the He matrix case are modeled by the macroscopic parameters of hydrostatic pressure and elastic stress. Under $D_{2}$ excitation the bubble acquires a very strong quadrupolar deformation. Due to the softness of the matrix, some He atoms can move into the dimples in the $M_{J}=3 / 2$ orbital or in between the two lobes of the $M_{J}=1 / 2$ orbital and fill all space available therein. As discussed in Sec. 5, these atoms are attracted by the van der Waals force and form a bound or quasibound complex, called exciplex. The resulting fluorescence spectrum (Fig. 21) is very similar in its structure, spectral width and shift to the one observed in [261-264] for alkali metals. In those experiments only visible and near infrared $(\lambda \leq 1000 \mathrm{~nm})$ fluorescence was studied, so that some spectral features might have escaped from observation. Similar results were obtained in $[286,273,287,275]$ for silver (Fig. 38).

Lifetime studies were reported for Ag [273,287], K [262], and Na [261]. In agreement with Eq. 34, the lifetime increases with the fluorescence wavelength, significantly exceeding the free atomic lifetime $\tau_{\text {free }}$. The effect is most pronounced in Ag-doped Xe $\left(\lambda_{\text {free }}=328 \mathrm{~nm}\right.$, $\tau_{\text {free }}=6.5 \mathrm{~ns}$ ), where two fluorescence maxima are observed at 550 and $680 \mathrm{~nm}$ (Fig. 38) with lifetimes of 28 and 58 ns, respectively. Applying Eq. 34 one can show that the transition 
dipole moment of a $\mathrm{Ag}$ atom stays practically unchanged, similar to the $\mathrm{Cs}^{*} \mathrm{He}_{7}$ exciplex (Sec. 8.2). In alkali-doped matrices the observed lifetimes are considerably shorter than predicted by Eq. 34. This may be due to a modification of the transition dipole moment, to the quenching of the radiating species by the formation of trapping sites of lower energy, or to the formation of exciplexes whose fluorescence is further redshifted, as observed for the $\mathrm{Cs}^{*} \mathrm{He}_{2}$ exciplex.

$\mathrm{Cs}^{*} \mathrm{He}$ exciplexes and relaxed trapping sites of excited alkali or silver atoms in rare gas matrices are formed by the same physical mechanism and their fluorescence spectra possess the common features of large spectral widths, large redshifts, and enhanced lifetimes. However, in the case of $\mathrm{Cs}$ in condensed He, the bubble relaxation can also proceed along the radial (breathing) coordinate which does not contribute to the Jahn-Teller splitting. This is a channel of minor importance under the $D_{2}$ excitation, but becomes dominant under the $D_{1}$ excitation. In the resulting equilibrium configuration of the trapping site (expanded atomic bubble) the ground state is not strongly perturbed and the transition is only weakly blueshifted and much less broadened than in absorption (see Fig. 19). The corresponding lifetime is very close to the lifetime $\tau_{\text {free }}$ of the free atom (Fig. 24). Such a fluorescence component is not present in heavier rare-gas matrices doped either with alkali or noble metal atoms. It is particularly important since it is used for monitoring optical pumping of implanted Cs atoms and for optical detection of magnetic resonance, as discussed in Sec. 9.1.

\subsection{Trapping site relaxation}

The dynamics of trapping site relaxation following optical excitation of the impurity atom have addressed in experiments [288,289] applying the technique of ultrafast pump-probe laser spectroscopy to Ag-doped solid Xe. A first (pump) laser pulse excites the $5 S \rightarrow 5 P$ transition, and the second (probe) pulse is used to measure the absorption at the same wavelength that is modified by the trapping site relaxation. For the $M_{J}$ components whose energy is lowered in a distorted trapping site, relaxation occurs within 100 fs after the excitation and cannot be resolved in the experiments. However, for the excited state that has a minimum in the almost undistorted trapping site, cage oscillations with a period of 1.3 ps have been detected. A strong coupling to the lower lying state results in a radiationless population decay of the oscillating state with a $270 \mathrm{fs}$ decay time. In another version of the same experiment a probe pulse of longer wavelength was used to drive the population from the relaxed states back to the ground state. However, these laser pulses also induce transitions from $5 P$ to higher lying states which are not characterized in the matrix and make the interpretation more difficult.

Ultrafast pump-probe experiments have also been reported for NO molecules in solid argon and neon [290-292]. Calculations [293,294] show that this relatively small molecule occupies a substitution site in the matrix, in the same way as alkali and noble metal atoms do. The molecular orbital of the lowest excited state $A^{2} \Sigma^{+}$is nearly spherical and its repulsive interaction with the surrounding rare gas atoms leads to a symmetric expansion of the cage that closely resembles the atomic bubble in condensed He. The first laser pulse excites the $A^{2} \Sigma^{+}$state, and the second wavelength-tunable pulse drives transitions to higher lying states, whose spectroscopy in the rare gas matrices is well established [295]. The experiments 
of [290-292] and molecular dynamics calculations of [293,294] reveal a very fast expansion of the cage following the excitation of the molecule that occurs on a sub-picosecond time scale. This initial expansion is followed by transient oscillations of the bubble around the new equilibrium configuration which are damped after approximately one period, i.e., 2-3 ps after the excitation.

No corresponding studies on atomic/molecular dopants in solid He have been reported so far. The only study of bubble dynamics [166,172] has been carried out in superfluid He, with $\mathrm{He}_{2}^{*}$ excimers, prepared in the metastable $a^{3} \Sigma_{u}$ state by a multistep excitation process including the ionization of $\mathrm{He}$ in the strong electric field of a femtosecond laser pulse, followed by dimerization and recombination of the molecular ions with electrons. The calculations $[296,92]$ show that the metastable excimer resides in a spherical bubble with a radius $R_{b}=6.9 \AA$. The bubble dynamics after two-photon excitation of the excimer into a higher-lying electronic state $d^{3} \Sigma_{u}$ by another laser pulse (pump) has also been studied. The probe pulse used in the experimental configuration has the same wavelength as the pump and produces a single-photon ionization from the $d^{3} \Sigma_{u}$ state. A decrease of fluorescence at the $d^{3} \Sigma_{u} \rightarrow b^{3} \Pi_{g}$ transition is observed as a function of the pump-probe delay. Those experiments have also revealed transient bubble oscillations damped after approximately one period. The oscillation period depends on He temperature and pressure and lies in the range of 100-200 ps, two orders of magnitude longer than in solid rare gas matrices. Applying a hydrostatic model, the authors attribute the damping either to the viscosity of the normal fluid fraction or to the emission of sound waves. The most recent time-dependent density functional theory calculations [297] have shown that these bubble dynamics are extremely sensitive to the long-range parts of the $\mathrm{He}_{2}^{*}$-He pair potentials which presently can not be calculated with sufficient precision.

\subsection{Magnetic studies}

It is very difficult to distinguish between the dynamic Jahn-Teller effect and the influence of anisotropic (static) crystal field effects, based on optical spectra only. In the heavier rare gases additional information has been obtained from MCD and ESR studies.

ESR spectra of Li, Na, K, and Rb in solid Ar, Kr, and Xe were reported in [268,284,298301] with techniques very close to the ones used in studies of atomic hydrogen in matrices, as well as in impurity-helium solids (see Sec. 10.2.5). Li, Na, and $\mathrm{K}$ atoms have a nuclear spin equal to $3 / 2$ and their ESR spectra therefore consist of four components (Fig. 39). The nuclear spin of $\mathrm{Rb}$ and $\mathrm{Cs}$ atoms is equal to $5 / 2$ and $7 / 2$, and the corresponding ESR spectra have 6 and 8 components, respectively. For Cs, only a part of the octet structure has been observed experimentally [298].

The theoretical models developed in [298,302-304] for hydrogen and alkali atoms in rare gas matrices predicted modifications of the electronic $g$ factor and of the hyperfine coupling constant $A$ due to the impurity-matrix interaction. Three effects leading to such modifications were considered: attractive van der Waals interactions, repulsive Pauli exclusion forces, and spin-orbit interaction in the $S$ state of the impurity atom perturbed by an admixture of $P$ orbitals of neighboring matrix atoms. The latter effect appears due to the overlap of the impurity valence electron's wavefunction with those of rare-gas atoms. According to 


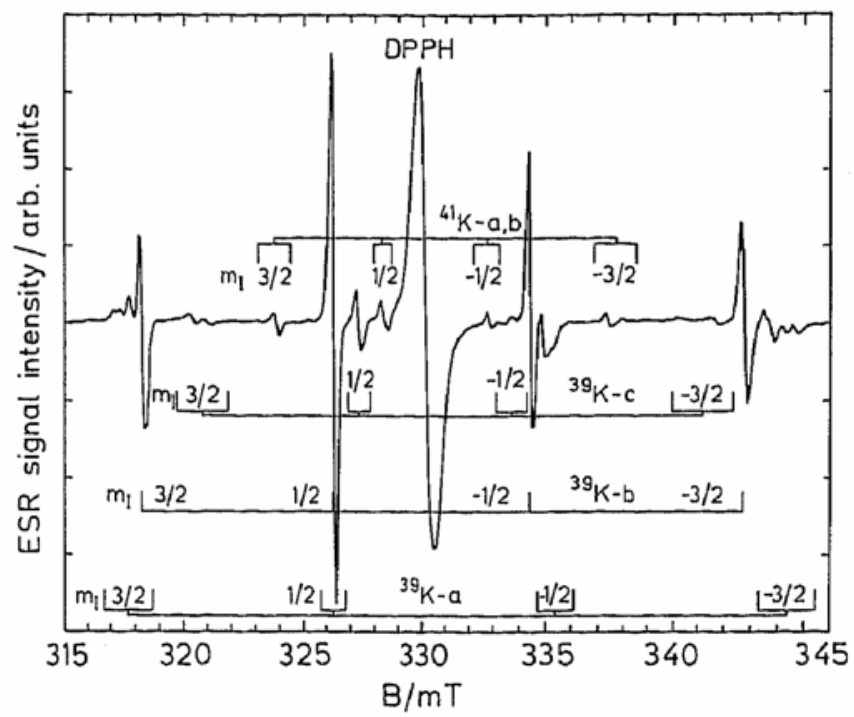

Fig. 39. ESR spectrum of potassium atoms isolated in solid argon, taken from [268]. The spectrum consists of quartet structures corresponding to ${ }^{41} \mathrm{~K}$ and to the three different types of trapping sites of ${ }^{39} \mathrm{~K}$ (labeled "a", "b", and "c"). The strongest feature in the center is from the calibration probe. Reproduced from [268] with kind permission of Springer Science and Business Media.

$[303,304]$ it dominates the $g$ factor shift, which is always negative, and does not affect $A$. The van der Waals and Pauli exclusion forces mostly affect the hyperfine coupling constant $A$ by changing the electronic density at the nucleus. The van der Waals force moves the electron density out of the nucleus and thus decreases the hyperfine coupling constant, while the Pauli exclusion force works in the opposite direction by compressing the electron wavefunction onto the nucleus.

The experimental results of $[268,284,298-301]$ confirm the theoretical predictions. Almost all measurements show very small $\left(\lesssim 10^{-3}\right)$ negative shifts of the $g$ factors, while the hyperfine constants $A$ are much more affected (up to 30\%). The latter either increases or decreases, depending on the trapping site. In agreement with the results of optical absorption studies, multiple trapping sites present within the same sample can be distinguished by differences of their hyperfine splittings and $g$ factors. As a rule, large positive shifts of the hyperfine frequency are accompanied by a large decrease of the $g$ factor. Such spectra are attributed to tighter trapping sites, in which the overlap of the orbitals of the impurity and matrix atoms is large, resulting in their mixing and in the compression of the impurity electron onto the nucleus. It has also been shown for Rb [300] and $\mathrm{K}$ [268] in solid Ar, that the strongly blueshifted optical absorption spectrum (the so-called blue triplet) originates from the same type of the trapping site. Conversely, ESR spectra with a smaller decrease of the $g$ value and a negative shift of the hyperfine frequency belong to loose trapping sites, in which the orbital overlap is small and where the van der Waals interaction dominates. This type of trapping site produces almost unshifted optical absorption spectra in experiments on $\mathrm{K}$ in solid $\operatorname{Ar}[268]$.

Most rare gas atoms have no nuclear spin. Only xenon has two isotopes ${ }^{129} \mathrm{Xe}$ and ${ }^{131} \mathrm{Xe}$ (nuclear spins $1 / 2$ and $3 / 2$ respectively) that have a significant relative abundances of $26 \%$ and $21 \%$, respectively. In Xe matrices the electron spin of the impurity atom interacts with 
nearby magnetic nuclei that results in an additional substructure of each ESR peak. This structure was investigated experimentally in isolated alkali [284] and noble metal [305] atoms.

Electron spin relaxation times were measured for $\mathrm{K}, \mathrm{Li}$, and $\mathrm{Ag}$ atoms in $\mathrm{Ar}$ matrices $[268,301]$ from studies of ESR saturation in strong rf fields. The longitudinal relaxation time $\mathrm{T}_{1}$ is on the order of $10 \mu \mathrm{s}$, whereas the transverse relaxation time is $\mathrm{T}_{2} \simeq 0.1 \mu \mathrm{s}$, i.e., orders of magnitude lower than the $\mathrm{T}_{1}$ and $\mathrm{T}_{2}$ times of alkali atoms in condensed He. The authors of [301] suggest a relaxation mechanism due to vibrations of lattice atoms that produce an oscillating overlap between the impurity and the matrix atoms' orbitals, which in turn results in variations of the hyperfine coupling constant of the impurity atom.

\section{Summary}

In this paper we have presented a comprehensive review of experimental and theoretical studies of the defects produced by atomic and simple molecular impurities in liquid and solid He. Most of the reported studies dealt with superfluid He, while for solid He only a few publications exist besides our own. The results of those studies show that due to the quantum nature of solid He the structure and the properties of the defects are very similar to those in liquid He. From a technical point of view the main advantage of the solid He matrix is the much longer residence time of the implanted species. In addition, in solid He a variety of new phenomena appear due to the anisotropy of the matrix, which is absent in the liquid phase. We have shown that such phenomena can be traced back to the macroscopic elastic properties of the He crystals.

Another group of new phenomena appears at the melting point of solid He, when liquid and solid helium are in equilibrium with each other. Under those conditions the macroscopic properties of the matrix become sensitive to the presence of charged impurities. The investigation of such systems was started only recently and has already brought spectacular results.

The interaction between the impurity atom and the host atoms in condensed helium is of a similar nature as in heavier rare gases, which have been and are being used in great number of matrix isolation studies. However, the particular properties of condensed helium as being quantum liquid/solid make it possible to use a very simple and instructive theoretical tool - the atomic bubble model for the defect description. This model combines classical hydrodynamics of a continuous medium with the quantum mechanical description of individual atoms and gives qualitative explanations to a number of complicated manybody phenomena observed in experiments. Moreover, the calculations based on the bubble model without any free parameters produce results that are in quantitative agreement with the experimental ones. This is a remarkable result considering the simplicity of the model.

The bubble model becomes invalid if the interaction of the impurity particle with helium is strongly anisotropic. This occurs typically for electronic states of the impurities with $p$ type orbitals. Such impurities form bound or quasibound complexes with one or several He atoms, so-called exciplexes. For their description another simple model can be applied that is based on impurity-helium pair potentials and that assumes that the complex structure is practically not affected by the surrounding He. 


\section{Acknowledgements}

The research work presented above is the fruit of more than a decade of experimental and theoretical efforts carried out by a number of undergraduate students, $\mathrm{Ph}$. D. students, and postdocs, and with the invaluable support of mechanical and electronics technicians and engineers. All their individual contributions are acknowledged. The doctoral thesis works that have emerged from this line of research were presented by Markus Arndt [306] and Stephen Lang [307] at the Ludwig-Maximilians Universität in Munich, by Taro Eichler [308] at the Friedrich-Wilhelms-Universität in Bonn and more recently by Daniel Nettels [309], Reinhard Müller-Siebert [21], Simone Ulzega [200], and Adrian Hofer [310] at the University of Fribourg. One of us (A. W.) acknowledges his colleague and friend Sergei Kanorsky who submitted the original EDM proposal to the Max-Planck-Institute for Quantum Optics and with whom he initiated the first heroic steps of this research.

The research was funded partly by the individual host institutions and by the national funding agencies in Germany (Deutsche Forschungsgemeinschaft, DFG) and in Switzerland (Swiss National Science Foundation, SNF). We particularly acknowledge the support of SNF by the grants 21-59451.99, 20-67008.01, and 200020-103864.

\section{References}

[1] S. Kanorsky, A. Weis, Atoms in nano-cavities, in: M. Ducloy, D. Bloch (Eds.), Quantum optics of confined systems, Kluwer Academic Publishers, 1996, pp. 367-393, NATO ASI series, Series E, Applied Sciences, vol. 413.

[2] S. I. Kanorsky, A. Weis, Optical and magneto-optical spectroscopy of point defects in condensed Helium, Academic Press, 1998, Advances in Atomic, Molecular and Optical Physics, vol. 38, B. Bederson, H. Walther.

[3] P. Moroshkin, A. Hofer, S. Ulzega, A. Weis, Spectroscopy of atomic and molecular defect in solid ${ }^{4}$ He using optical, microwave, radio frequency, magnetic and electric fields, Low Temp. Phys. 32 (11) (2006) 981-998, (Fiz. Nizk. Temp. 32 (11) (2006) 1297-1319).

[4] B. Tabbert, H. Günther, G. zu Putlitz, Optical investigation of impurities in superfluid helium $\left({ }^{4} \mathrm{He}\right)$, J. Low Temp. Phys. 109 (5-6) (1997) 653-707.

[5] J. P. Toennies, A. F. Vilesov, Spectroscopy of atoms and molecules in liquid helium, Annu. Rev. Phys. Chem. 49 (1998) $1-41$.

[6] F. Stienkemeier, A. F. Vilesov, Electronic spectroscopy in He droplets, J. Chem. Phys. 115 (22) (2001) 10119-10137.

[7] J. P. Toennies, A. F. Vilesov, Superfluid helium droplets: a unique cold nanomatrix for molecules and molecular complexes, Angewandte Chemie 43 (2004) 2622-2648.

[8] F. Stienkemeier, K. K. Lehmann, Spectroscopy and dynamics in helium nanodroplets, J. Phys. B 39 (2006) R127-R166.

[9] C. Crepin-Gilbert, A. Tramer, Photophysics of metal atoms in rare-gas complexes, clusters and matrices, Int. Reviews in Physical Chemistry 18 (4) (1999) 485-556.

[10] E. P. Bernard, R. E. Boltnev, V. V. Khmelenko, V. Kiryukhin, S. I. Kiselev, D. M. Lee, Impurity-helium solids: Chemistry and physics at $1.5 \mathrm{~K}$, J. Low Temp. Phys. 134 (1-2) (2004) 133-143.

[11] L. P. Mezhov-Deglin, Impurity nanocluster structures in liquid helium, Physics-Uspekhi 48 (10) (2005) 1061-1070, (Usp. Fiz. Nauk, 175 (10) (2005) 1115-1124).

[12] V. V. Khmelenko, H. Kunttu, D. M. Lee, Recent progress in studies of nanostructured impurity-helium solids, J. Low Temp. Phys. 148 (2007) 1-31.

[13] E. B. Gordon, A. F. Shestakov, Localization of helium around microscopic impurities in liquid helium, Low Temp. Phys. 26 (1) (2000) 1-23, (Fiz. Nizk. Temp., 26 (1) (2000) 5-33).

[14] H. R. Glyde, Excitations in liquid and solid helium, Clarendon Press, Oxford, 1994.

[15] R. A. Aziz, A. R. Janzen, M. R. Moldover, Ab initio calculations for helium: A standard for transport property measurements, Phys. Rev. Lett. 74 (9) (1995) 1586-1589.

[16] J. Wilks, The properties of liquid and solid helium, Clarendon Press, Oxford, 1967.

[17] R. H. Crepeau, O. Heybey, D. M. Lee, S. A. Strauss, Sound propagation in hcp solid helium crystals of known orientation, Phys. Rev. A 3 (1971) 1162-1174.

[18] D. S. Greywall, Elastic constants of bcc ${ }^{4} \mathrm{He}$, Phys. Rev. B 13 (1976) 1056-1068.

[19] E. Kim, M. H. W. Chan, Observation of superflow in solid helium, Science 305 (2004) 1941-1944.

[20] Proceedings of 7th conference on cryocrystals and quantum crystals, to be published in Low Temp. Phys. 
[21] R. Müller-Siebert, Optimierung und Charakterisierung eines tieftemperatur Spektrometers zur Untersuchung von magneto und elektro optischen effekten an Alkaliatomen in He Kristallen., Ph.D. thesis, University of Fribourg, Switzerland, unpublished (2002).

[22] D. L. Elwell, H. Meyer, Molar volume, coefficient of thermal expansion, and related properties of liquid ${ }^{4}$ He under pressure, Phys. Rev. 164 (1) (1967) 245-255.

[23] J. F. Jarvis, D. Ramm, H. Meyer, Measurement of $(\partial p / \partial t)_{v}$ and related properties in solidified gases. I. Solid He, Phys. Rev. 170 (1) (1968) 320-327.

[24] D. O. Edwards, R. C. Pandorf, Heat capacity and other properties of body-centered cubic ${ }^{4}$ He, Phys. Rev. 144 (1) (1966) $143-151$.

[25] E. R. Grilly, R. L. Mills, PVT relations in ${ }^{4}$ He near the melting curve and the $\lambda$-line, Ann. Phys. 18 (1962) $250-263$.

[26] J. Jortner, N. R. Kestner, S. A. Rice, M. H. Cohen, Study of the properties of an excess electron in liquid helium: I. The nature of the electron-helium interactions, J. Chem. Phys. 43 (8) (1965) 2614-2625.

[27] K. Hiroike, N. R. Kestner, S. A. Rice, J. Jortner, Study of the properties of an excess electron in liquid helium. II. A refined description of configuration changes in the liquid, J. Chem. Phys. 43 (8) (1965) 2625-2632.

[28] B. E. Springett, M. H. Cohen, J. Jortner, Properties of an excess electron in liquid helium: The effect of pressure on the properties of the negative ion, Phys. Rev. 159 (1) (1967) 183-190.

[29] B. E. Springett, M. H. Cohen, J. Jortner, Stability criterion for the localization of an excess electron in a nonpolar fluid, J. Chem. Phys. 48 (6) (1968) 2720-2731.

[30] M. Rosenblit, J. Jortner, Dynamics of the formation of an electron bubble in liquid helium, Phys. Rev. Lett. 75 (22) (1995) 4079-4082.

[31] M. Rosenblit, J. Jortner, Dynamics of excess electron localization in liquid helium and neon, J. Phys. Chem. A 101 (1997) $751-757$.

[32] J. Eloranta, V. A. Apkarian, A time dependent density functional treatment of superfluid dynamics: Equilibration of the electron bubble in superfluid ${ }^{4} \mathrm{He}$, J. Chem. Phys. 117 (22) (2002) 10139-10150.

[33] V. Grau, M. Barranco, R. Mayol, M. Pi, Electron bubbles in liquid helium: Density functional calculations of infrared absorption spectra, Phys. Rev. B 73 (6) (2006) 064502.

[34] R. M. Ostermeier, Pressure dependence of charge carrier mobilities in superfluid helium, Phys. Rev. A 8 (1) (1973) $514-529$.

[35] B. Brody, Charge-carrier mobilities in superfluid helium under pressure, Phys. Rev. A 11 (1) (1975) 170-177.

[36] W. B. Fowler, D. L. Dexter, Electronic bubble states in liquid helium, Phys. Rev. 176 (1) (1968) $337-343$.

[37] B. DuVall, V. Celli, Electronic properties of negative ions in liquid helium, Phys. Rev. 180 (1) (1969) 276-286.

[38] T. Miyakawa, D. L. Dexter, Interpretation of photoejection experiments and the well depth of electronic bubbles in liquid helium, Phys. Rev. A 1 (2) (1970) 513-518.

[39] A. P. Hickman, N. F. Lane, Localized excited states of helium in liquid helium, Phys. Rev. Lett. 26 (20) (1971) 1216-1219.

[40] W. Steets, A. P. Hickman, N. F. Lane, Theoretical description of pressure shifts and quenching of excited atomic states in liquid helium, Chem. Phys. Lett. 28 (1) (1974) 31-34.

[41] A. P. Hickman, W. Steets, N. F. Lane, Nature of excited helium atoms in liquid helium: A theoretical model, Phys. Rev. B 12 (9) (1975) 3705-3717.

[42] H. Bauer, M. Beau, B. Friedl, C. Marchand, K. Miltner, H. J. Reyher, Laser spectroscopy of alkaline earth atoms in He II, Phys. Lett. A 146 (3) (1990) 134-140.

[43] G. zu Putlitz, M. R. Beau, Dye laser spectroscopy of isolated atoms and ions in liquid helium, Topics in Applied Physics 70 (1992) 237-247.

[44] T. Kinoshita, K. Fukuda, Y. Takahashi, T. Yabuzaki, Optical properties of impurity atoms in pressurized superfluid helium, Z. Phys. B 98 (1995) 387-390.

[45] T. Kinoshita, K. Fukuda, Y. Takahashi, T. Yabuzaki, Optical properties of alkali-metal atoms in pressurized liquid helium, Phys. Rev. A 52 (4) (1995) 2707-2716.

[46] T. Kinoshita, K. Fukuda, T. Yabuzaki, Doubly shaped $\mathrm{D}_{2}$ excitation spectra of Cs and Rb atoms in superfluid helium due to a quadrupole bubble surface oscillation, Phys. Rev. B 54 (9) (1996) 6600-6607.

[47] G. DeToffol, F. Ancilotto, F. Toigo, Excited states of alkali atoms in liquid ${ }^{4}$ He from density functional calculations, J. Low Temp. Phys. 102 (5-6) (1996) 381-410.

[48] A. Hofer, P. Moroshkin, S. Ulzega, D. Nettels, R. Müller-Siebert, A. Weis, $6 \mathrm{~S}_{1 / 2}-6 \mathrm{P}_{1 / 2}$ transition of Cs atoms in cubic and hexagonal solid ${ }^{4}$ He, Phys. Rev. A 76 (2007) 022502.

[49] J. Pascale, Pseudopotential molecular-structure calculations for NaHe and CsHe, Phys. Rev. A 26 (6) (1982) 3709-3712.

[50] J. Pascale, Use of l-dependent pseudopotential in the study of alkali-metal-atom-he systems. The adiabatic molecular potentials, Phys. Rev. A 28 (2) (1983) 632-644.

[51] S. Kanorsky, A. Weis, M. Arndt, R. Dziewior, T. Hänsch, Pressure shift of atomic resonance lines in liquid and solid helium, Z. Phys. B 98 (1995) 371-376.

[52] J. Z. Tang, M. Kimura, I. Shimamura, Absorption and emission spectra of alkaline-earth atoms in liquid helium: a theoretical study, Chem. Phys. Lett. 256 (1996) 327-333.

[53] I. Baumann, A. Breidenassel, C. Zühlke, A. Kasimov, G. zu Putlitz, I. Reinhard, K. Jungmann, Pressure dependence of the $\mathrm{Mg} 3 \mathrm{~s} 4 \mathrm{~s}{ }^{3} S_{1} \rightarrow 3 \mathrm{~s} 3 \mathrm{p}{ }^{3} P_{0,1,2}$ transition in superfluid ${ }^{4} \mathrm{He}$, Eur. Phys. J. D. 12 (2000) $117-122$.

[54] Y. Moriwaki, K. Inui, K. Kobayashi, F. Matsushima, N. Morita, Laser spectroscopic study of Mg atoms in pressurized liquid helium, J. Mol. Structure 786 (2006) 112-117. 
[55] M. Beau, H. Günther, G. zu Putlitz, B. Tabbert, Atoms and ions in superfluid helium II. Theoretical considerations, Z. Phys. B 101 (1996) 253-262.

[56] Y. Moriwaki, N. Morita, Laser spectroscopic investigation of Ca atoms in liquid helium, Eur. Phys. J. D 33 (2005) $323-327$.

[57] S. I. Kanorsky, M. Arndt, R. Dziewior, A. Weis, T. W. Hänsch, Pressure shift and broadening of the resonance line of barium atoms in liquid helium, Phys. Rev. B 50 (9) (1994) 6296-6302.

[58] Y. Moriwaki, N. Morita, Spectroscopic studies on Yb+ ions in liquid helium, Eur. Phys. J. D 13 (2001) 11-20.

[59] Q. Wang, M. H. Alexander, Path integral Monte Carlo simulation of the absorption spectra of an Al atom embedded in helium, J. Chem. Phys. 123 (2005) 134319.

[60] V. Ludwig, P. K. Mukherjee, K. Coutinho, S. Canuto, Spectral shift of sodium in a liquid-helium environment: A sequential Monte Carlo time-dependent density-functional study, Phys. Rev. A 72 (2005) 062714.

[61] P. B. Lerner, M. B. Chadwick, I. M. Sokolov, Inhomoheneous broadening of electronic transitions in a liquid helium bubble: The role of shape fluctuations, J. Low Temp. Phys. 90 (304) (1993) 319-330.

[62] H. J. Maris, W. Guo, The shape of electron bubbles in liquid helium and the line width of optical transitions, J. Low Temp. Phys. 137 (3-4) (2004) 491-507.

[63] M. H. Cohen, J. Jortner, Electron cavity formation in solid helium, Phys. Rev. 180 (1) (1969) $238-241$.

[64] A. Y. Parshin, S. V. Pereverzev, Spectroscopic study of excess electrons in liquid helium, J. Exp. Theor. Phys. 74 (1992) 68, (Zh. Eksp. Teor. Fiz., 101 (1) (1992) 126-142).

[65] C. C. Grimes, G. Adams, Infrared-absorption spectrum of the electron bubble in liquid helium, Phys. Rev. B 45 (5) (1992) $2305-2310$.

[66] A. I. Golov, L. P. Mezhov-Deglin, Measurement of the infrared absorption spectrum by negative charges in solid helium, J. Exp. Theor. Phys. Lett. 56 (10) (1992) 514-518, (Pis'ma Zh. Eksp. Teor. Fiz., 56 (10) (1992) 527-531).

[67] S. I. Kanorsky, M. Arndt, R. Dziewior, A. Weis, T. W. Hänsch, Optical spectroscopy of atoms trapped in solid helium, Phys. Rev. B 49 (5) (1994) 3645-3647.

[68] P. Moroshkin, A. Hofer, D. Nettels, S. Ulzega, A. Weis, Cs` He ${ }_{n}$ exciplexes in solid ${ }^{4}$ He, J. Chem. Phys. 124 (2006) 024511.

[69] T. Paszkiewicz, S. Wolski, Young's and shear moduli and Poisson's ratio for elastic media of high and middle symmetry, Phys. Stat. Sol. B 244 (3) (2007) 978-987.

[70] S. Kanorsky, S. Lang, T. Eichler, K. Winkler, A. Weis, Quadrupolar deformations of atomic bubbles in solid ${ }^{4}$ He, Phys. Rev. Lett. 81 (2) (1998) 401-404.

[71] P. Moroshkin, A. Hofer, A. Weis, Deformed atomic bubbles in solid ${ }^{4}$ He, to be published.

[72] S. Ulzega, A. Hofer, P. Moroshkin, A. Weis, Reconciliation of experimental and theoretical electric tensor polarizabilities of the cesium ground state, Europhys. Lett. 76 (2006) 1074-1080.

[73] S. Lang, S. Kanorsky, M. Arndt, S. B. Ross, T. W. Hänsch, A. Weis, The hyperfine structure of Cs atoms in the bcc phase of solid He, Europhys. Lett. 30 (4) (1995) 233-237.

[74] Y. Takahashi, K. Fukuda, T. Kinoshita, T. Yabuzaki, Sublevel spectroscopy of alkali atoms in superfluid helium, Z. Phys. B 98 (1995) 391-393.

[75] H. Maris, On the fission of elementary particles and the evidence for fractional electrons in liquid helium, J. Low Temp. Phys. 120 (3-4) (2000) 173-204.

[76] H. Maris, D. Konstantinov, Bubbles in liquid helium containing electrons in excited states, J. Low Temp. Phys. 121 (5-6) (2000) 615-620.

[77] H. J. Maris, Properties of electron bubbles in liquid helium, J. Low Temp. Phys. 132 (1-2) (2003) 77-95.

[78] H. J. Maris, W. Guo, Calculation of the shape of S-state electron bubbles in liquid helium, J. Low Temp. Phys. 148 (2007) $207-211$

[79] P. Grinfeld, H. Kojima, Instability of the 2S electron bubbles, Phys. Rev. Lett. 91 (10) (2003) 105301.

[80] R. Jackiw, C. Rebbi, J. R. Schrieffer, Fractional electrons in liquid helium, J. Low Temp. Phys. 122 (5-6) (2001) 587-589.

[81] A. I. Rae, W. F. Vinen, Electrino collapse, J. Low Temp. Phys. 123 (1-2) (2001) 1-5.

[82] V. Elser, Indivisibility of electron bubbles in helium, J. Low Temp. Phys. 123 (1-2) (2001) 7-23.

[83] B. Altschul, C. Rebbi, Analysis of a toy model of electron splitting, Phys. Rev. A 69 (3) (2004) 032111.

[84] L. Lentovaara, J. Eloranta, One- and two-electron bubbles in superfluid ${ }^{4}$ He, J. Low. Temp. Phys. 148 (2007) $43-52$.

[85] Y. Moriwaki, N. Morita, Ultraviolet spectra of Mg in liquid helium, Eur. Phys. J. D 5 (1999) 53-57.

[86] V. Celli, M. Cohen, M. J. Zuckerman, Vibrational modes of a negative ion in liquid helium, Phys. Rev. 173 (1) (1968) $253-256$.

[87] I. A. Fomin, Absorption and scattering of light by excess electrons in liquid helium, J. Exp. Theor. Phys. Lett. 6 (6) (1967) 196-198, (Pis'ma Zh. Eksp. Teor. Fiz., 6 (6), 715-718 (1967)).

[88] W. Guo, H. J. Maris, Calculation of the cross-section for optical transitions of an electron bubble to D states, J. Low Temp. Phys. 148 (2007) 213-217.

[89] W. S. Dennis, E. Durbin, W. A. Fitzsimmons, O. Heybey, G. K. Walters, Spectroscopic identification of excited atomic and molecular states in electron-bombarded liquid helium, Phys. Rev. Lett. 23 (19) (1969) $1083-1086$.

[90] A. Trottier, A. I. Jirasek, H. F. Tiedje, R. L. Brooks, Spectra of liquid helium and hydrogen-doped liquid helium, Phys. Rev. A 61 (5) (2000) 052504.

[91] T. Kiljunen, L. Lehtovaara, H. Kunttu, J. Eloranta, Solvation of triplet Rydberg states of molecular hydrogen in superfluid helium, Phys. Rev. A 69 (2004) 012506.

[92] J. Eloranta, N. Schwentner, V. A. Apkarian, Structure and energetics of He ${ }_{2}^{*}$ bubble-states in superfluid ${ }^{4}$ He, J. Chem. Phys. 116 (10) (2002) 4039-4053. 
[93] J. Dupont-Roc, Excited p-states of alkali atoms in liquid helium, Z. Phys. B 98 (1995) 383-386.

[94] T. Kinoshita, K. Fukuda, T. Matsuura, T. Yabuzaki, Pressure-dependent quenching of Rb 5P states in liquid helium, Phys. Rev. A 53 (6) (1996) 4054-4063.

[95] J. Reho, C. Callegari, J. Higgins, W. E. Ernst, K. K. Lehmann, G. Scoles, Spin-orbit effects in the formation of the Na-He excimer on the surface of He clusters, Faraday Discuss. 108 (1997) 161-174.

[96] J. Reho, J. Higgins, C. Callegari, K. K. Lehmann, G. Scoles, Alkali-helium exciplex formation on the surface of helium nanodroplets. I. Dispersed emission spectroscopy, J. Chem. Phys. 113 (21) (2000) 9686-9693.

[97] J. Reho, J. Higgins, K. K. Lehmann, G. Scoles, Alkali-helium exciplex formation on the surface of helium nanodroplets. II. A time-resolved study, J. Chem. Phys. 113 (21) (2000) 9694-9701.

[98] F. R. Brühl, R. A. Trasca, W. E. Ernst, Rb-He exciplex formation on helium nanodroplets, J. Chem. Phys. 115 (22) (2001) 10220-10224.

[99] K. Enomoto, K. Hirano, M. Kumakura, Y. Takahashi, T. Yabuzaki, Emission spectra of Cs-He excimers in cold helium gas, Phys. Rev. A 66 (2002) 042505.

[100] J. L. Persson, Q. Hui, Z. J. Jakubek, M. Nakamura, M. Takami, Formation of $\mathrm{AgHe}_{2}$ exciplex in liquid helium, Phys. Rev. Lett. 76 (9) (1996) 1501-1504.

[101] Z. J. Jakubek, M. Takami, Ab initio studies of AgHe exciplex, Chem. Phys. Lett. 265 (1997) 653-659.

[102] Y. Fukuyama, Y. Moriwaki, Y. Matsuo, Laser-induced fluorescence spectra of $\mathrm{Ba}^{+*}{ }_{-} \mathrm{He}$ exciplexes produced in cold He gas, Phys. Rev. A 69 (2004) 042505.

[103] Y. Fukuyama, Y. Moriwaki, Y. Matsuo, Formation and dissociation of $\mathrm{Ba}^{+*}-\mathrm{He}$ exciplexes at 3-25 K, Phys. Rev. A 75 (2007) 032725.

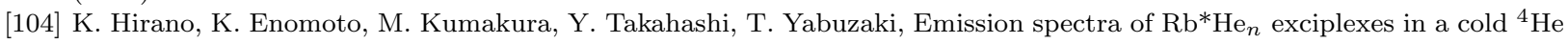
gas, Phys. Rev. A 68 (2003) 012722.

[105] K. Enomoto, K. Hirano, M.Kumakura, Y. Takahashi, T. Yabuzaki, Emission spectra of alkali-metal (K, Na, Li)-He exciplexes in cold helium gas, Phys. Rev. A 69 (2004) 012501.

[106] T. Takayanagi, M. Shiga, Theoretical study on photoexcitation dynamics of the K atom attached to helium clusters and the solvation structure of $\mathrm{K}^{*} \mathrm{He}_{n}$ exciplexes, Phys. Chem. Chem. Phys. 6 (2004) 3241-3247.

[107] M. Zbiri, C. Daul, Investigating the $\mathrm{M}^{*} \mathrm{He}$ exciplexes, $\mathrm{M}=\{\mathrm{Li}, \mathrm{Na}, \mathrm{K}, \mathrm{Rb}, \mathrm{Cs}, \mathrm{Fr}\}$ : Density functional approach, J. Chem. Phys. 121 (23) (2004) 11625-11628.

[108] M. Zbiri, C. Daul, Theoretical investigation of the $\mathrm{Cs}^{*} \mathrm{He}_{n \geq 3}$ exciplexes, Phys. Lett. A 341 (2005) 170-176.

[109] M. Lehner, R. Xu, M. Jungen, The emission spectrum of the Li(2p)He $2: 1^{2} \Pi_{u}$ exciplex, J. Phys. B 38 (2005) $1235-1247$.

[110] M. Mella, M. C. Colombo, G. Morosi, Ground state and excitation dynamics in Ag doped helium clusters, J. Chem. Phys. 117 (21) (2002) 9695-9702.

[111] A. Wada, T. Takayanagi, M. Shiga, Theoretical simulations on photoexcitation dynamics of the silver atom embedded in helium clusters, J. Chem. Phys. 119 (11) (2003) 5478-5486.

[112] P. Moroshkin, D. Nettels, A. Hofer, S. Ulzega, A. Weis, Laser-induced formation and spectroscopy of $\mathrm{Cs}^{*} \mathrm{He}_{n}$ exciplexes in solid ${ }^{4} \mathrm{He}$, Proc. of SPIE 6257 (2006) 625706.

[113] A. Hofer, P. Moroshkin, S. Ulzega, A. Weis, $\mathrm{Rb}^{*} \mathrm{He}_{n}$ exciplexes in solid ${ }^{4} \mathrm{He}$, Phys. Rev. A 74 (2006) 032509.

[114] W. I. Glaberson, W. W. Johnson, Impurity ions in liquid helium, J. Low Temp. Phys. 20 (3-4) (1975) 313-338.

[115] H. Bauer, M. Hausmann, R. Mayer, H. J. Reyher, E. Weber, A. Winnacker, Implantation of impurity ions into He II for optical spectroscopy studies, Phys. Lett. A 110 (5) (1985) 279-282.

[116] H. J. Reyher, H. Bauer, C. Huber, R. Mayer, A. Schafer, A. Winnacker, Spectroscopy of barium ions in He II, Phys. Lett. A 115 (5) (1986) 238-244.

[117] H. Bauer, M. Beau, A. Bernhard, B. Friedl, H. J. Reyher, Implantation of atoms into liquid helium for the purpose of impurity spectroscopy, Phys. Lett. A 137 (4-5) (1989) 217-224.

[118] B. Tabbert, M. Beau, M. Foerste, H. Günther, C. Honninger, H. Hust, K. Layer, G. zu Putlitz, T. Schumacher, Optical studies of atoms and ions in superfluid helium using a ccd-camera system, Z. Phys. B 98 (1995) 399-404.

[119] M. Arndt, S. I. Kanorsky, A. Weis, T. W. Hänsch, Can paramagnetic atoms in superfluid helium be used to search for permanent electric dipole moments?, Phys. Lett. A 174 (1993) 298-303.

[120] H. Günther, M. Foerste, C. Honninger, G. zu Putlitz, B. Tabbert, Lifetime of metastable magnesium atoms in superfluid helium, Z. Phys. B 98 (1995) 395-398.

[121] A. Fujisaki, K. Sano, T. Kinoshita, Y. Takahashi, T. Yabuzaki, Implantation of neutral atoms into liquid helium by laser sputtering, Phys. Rev. Lett. 71 (7) (1993) 1039-1042.

[122] Y. Takahashi, K. Sano, T. Kinoshita, T. Yabuzaki, Spectroscopy of alkali atoms and molecules in superfluid helium, Phys. Rev. Lett. 71 (7) (1993) 1035-1038.

[123] T. Kinoshita, Y. Takahashi, T. Yabuzaki, Optical pumping and optical detection of the magnetic resonance of alkali-metal atoms in superfluid helium, Phys. Rev. B 49 (5) (1994) 3648-3651.

[124] J. H. M. Beijersbergen, Q. Hui, M. Takami, Spectroscopy and dynamics of trapped alkaline earth atoms in superfluid helium, Phys. Lett. A 181 (1993) 393-403.

[125] Q. Hui, J. L. Persson, J. H. M. Beijersbergen, M. Takami, Spectroscopy and dynamics of neutral atoms in superfluid helium, Z. Phys. B 98 (1995) 353-357.

[126] M. Arndt, R. Dziewior, S. Kanorsky, A. Weis, T. Hänsch, Implantation and spectroscopy of metal atoms in solid helium, Z. Phys. B 98 (1995) 377-381. 
[127] P. Moroshkin, A. Hofer, S. Ulzega, A. Weis, Impurity-stabilized solid ${ }^{4}$ He below the solidification point of pure helium, Nature Physics 3 (2007) 786-789.

[128] E. B. Gordon, L. P. Mezhov-Deglin, O. F. Pugachev, Stabilization of nitrogen atoms in superfluid helium, J. Exp. Theor. Phys. Lett. 19 (2) (1974) 63-65, Pis'ma Zh. Eksp. Teor. Fiz. 19 (2), 103-106 (1974).

[129] E. B. Gordon, V. V. Khmelenko, A. A. Pelmenev, E. A. Popov, O. F. Pugachev, Impurity-helium van der Waals crystals, Chem. Phys. Lett. 155 (3) (1989) 301-304.

[130] E. B. Gordon, A. Usenko, G. Frossati, Embedding impurities into solid helium, J. Low Temp. Phys. 130 (1-2) (2003) $15-23$.

[131] E. B. Gordon, Impurity condensation in liquid and solid helium, Low Temp. Phys. 30 (10) (2004) 756-762, (Fiz. Nizk. Temp. 30 (10) (2004) 1009-1018).

[132] D. Nettels, A. Hofer, P. Moroshkin, R. Müller-Siebert, S.Ulzega, A. Weis, Discovery of dumbbell-shaped $\mathrm{Cs}^{\star} \mathrm{He}_{n}$ exciplexes in solid ${ }^{4} \mathrm{He}$, Phys. Rev. Lett. 94 (2005) 063001.

[133] P. Moroshkin, A. Hofer, S. Ulzega, A. Weis, Spectroscopy of Rb 2 dimers in solid ${ }^{4}$ He, Phys. Rev. A 74 (2006) 032504.

[134] A. Hofer, P. Moroshkin, S. Ulzega, A. Weis, Lifetime of the $\mathrm{Cs}_{6} 6 \mathrm{P}_{1 / 2}$ state in bcc and hcp solid ${ }^{4}$ He, Eur. Phys. J. D 46 (1) (2008) 9-14.

[135] J. A. Northby, T. M. Sanders, Photoejection of electrons from bubble states in liquid helium, Phys. Rev. Lett. 18 (26) (1967) 1184-1186.

[136] C. C. Grimes, G. Adams, Infrared spectrum of the electron bubble in liquid helium, Phys. Rev. B 41 (10) (1990) 6366-6371.

[137] A. Y. Parshin, S. V. Pereverzev, Direct observation of optical absorption by excess electrons in liquid helium, J. Exp. Theor. Phys. Lett. 52 (5) (1990) 282-284, (Pis'ma Zh. Eksp. Teor. Fiz., 52 (5), 905-907 (1990)).

[138] A. I. Golov, L. P. Mezhov-Deglin, IR absorption spectrum of excess electrons in solid helium, Physica B $194-196$ (1994) 951-952.

[139] A. I. Golov, Spectroscopic study of injected electrons in solid helium, Z. Phys. B 98 (1995) 363-366.

[140] C. L. Zipfel, Ph.D. thesis, University of Michigan, unpublished (1969).

[141] D. Konstantinov, H. J. Maris, Detection of excited-state electron bubbles in superfluid helium, Phys. Rev. Lett. 90 (2) (2003) 025302.

[142] H. J. Maris, A. Ghosh, D. Konstantinov, M. Hirsh, Experiments to study the effect of light on electron bubbles in liquid helium, J. Low Temp. Phys. 134 (1-2) (2004) 491-507.

[143] A. Ghosh, H. J. Maris, Measurement of the lifetime of excited-state electron bubbles in superfluid helium, Phys. Rev. B $72(5)(2005) 054512$.

[144] J. Classen, C. K. Su, H. J. Maris, Observation of exploding electron bubbles in liquid helium, Phys. Rev. Lett. 77 (10) (1996) 2006-2008.

[145] J. Classen, C. K. Su, M. Mohazzab, H. J. Maris, Electrons and cavitation in liquid helium, Phys. Rev. B 57 (5) (1998) $3000-3010$

[146] J. C. Hill, O. Heybey, G. K. Walters, Evidence of metastable atomic and molecular bubble states in electron-bombarded superfluid liquid helium, Phys. Rev. Lett. 26 (20) (1971) 1213-1216.

[147] J. W. Keto, F. J. Soley, M. Stockton, W. A. Fitzsimmons, Dynamic properties of neutral excitations produced in electronbombarded superfluid helium. I. The $\mathrm{He}\left(2^{3} \mathrm{~S}\right)$ and $\mathrm{He}_{2}\left(\mathrm{a}^{3} \sigma\right)$ atomic and molecular metastable states, Phys. Rev. A 10 (3) (1974) 872-886.

[148] J. W. Keto, F. J. Soley, M. Stockton, W. A. Fitzsimmons, Dynamic properties of neutral excitations produced in electronbombarded superfluid helium. II. Afterglow fluorescence of excited helium molecules, Phys. Rev. A 10 (3) (1974) 887-896.

[149] F. J. Soley, W. A. Fitzsimmons, Pressure shifts and quenching of atomic and molecular states produced in electronbombarded liquid helium, Phys. Rev. Lett. 32 (18) (1974) 988-991.

[150] W. A. Fitzsimmons, Excited atomic and molecular states in liquid helium, in: J. Smith, K. Walters (Eds.), Atomic Physics 3, Plenum, New York, 1983, pp. 477-492.

[151] A. Bartelt, J. D. Close, F. Federmann, N. Quaas, J. P. Toennies, Cold metal clusters: Helium droplets as a nanoscale cryostat, Phys. Rev. Lett. 77 (17) (1996) 3525-3528.

[152] E. Loginov, M. Drabbels, Excited state dynamics of Ag atoms in helium nanodroplets, J. Phys. Chem. A 111 (2007) $7504-7515$.

[153] J. Reho, U. Merker, M. R. Radcliff, K. K. Lehmann, G. Scoles, Spectroscopy of Mg atoms solvated in helium nanodroplets, J. Chem. Phys. 112 (19) (2000) 8409-8416.

[154] J. Reho, U. Merker, M. R. Radcliff, K. K. Lehmann, G. Scoles, Spectroscopy and dynamics of Al atoms solvated in superfluid helium nanodroplets, J. Phys. Chem. A 104 (2000) 3620-3626.

[155] B. Tabbert, M. Beau, H. Günther, W. Haussler, C. Honninger, K. Meyer, B. Plagemann, G. zu Putlitz, Atoms and ions in superfluid helium I. Optical spectra of atomic and ionic impurities, Z. Phys. B 97 (1995) 425-432.

[156] M. Mella, G. Calderoni, F. Cargoni, Predicting atomic dopant solvation in helium clusters: The MgHe ${ }_{n}$ case, J. Chem. Phys. 123 (5) (2005) 054328.

[157] M. Melich, J. Dupont-Roc, P. Jacquier, Search for anisotropic effects of hcp solid helium on optical lines of cesium impurities, J. Low Temp. Phys. 150 (2008) 301-305.

[158] K. Ishikawa, A. Hatakeyama, K. Gosyono-o, S. Wada, Y. Takahashi, T. Yabuzaki, Laser spectroscopy of thulium atoms implanted in liquid and solid ${ }^{4} \mathrm{He}$, Phys. Rev. B 56 (2) (1997) 780-787.

[159] C. P. Schulz, P. Claas, F. Stienkemeier, Formation of K*He exciplexes on the surface of helium nanodroplets studied in real time, Phys. Rev. Lett. 87 (15) (2001) 153401. 
[160] G. Droppelmann, O. Bünermann, C. P. Schulz, F. Stienkemeier, Formation times of RbHe exciplexes on the surface of superfluid versus normalfluid helium nanodroplets, Phys. Rev. Lett. 93 (2) (2004) 023402.

[161] O. Bünermann, M. Mudrich, M. Weidemüller, F. Stienkemeier, Spectroscopy of Cs attached to helium nanodroplets, J. Chem. Phys. 121 (18) (2004) 8880-8886.

[162] N. Schwentner, E. E. Koch, J. Jortner, Electronic excitations in condensed rare gases, Springer, 1985, springer tracts in modern physics, vol. 107.

[163] M. Stockton, J. W. Keto, W. A. Fitzsimmons, Ultraviolet emission spectrum of electron-bombarded superfluid helium, Phys. Rev. Lett. 24 (12) (1970) 654-657.

[164] C. M. Surko, R. E. Packard, G. J. Dick, F. Reif, Spectroscopic study of the luminescence of liquid helium in the vacuum ultraviolet, Phys. Rev. Lett. 24 (12) (1970) 657-659.

[165] M. Stockton, J. W. Keto, W. A. Fitzsimmons, Ultraviolet emission spectrum of electron-bombarded superfluid helium, Phys. Rev. A 5 (1) (1972) 372-380.

[166] A. V. Benderskii, R. Zadoyan, N. Schwenter, V. A. Apkarian, Photodynamics in superfluid helium: Femtosecond laserinduced ionization, charge recombination, and preparation of molecular Rydberg states, J. Chem. Phys. 110 (3) (1999) $1542-1557$.

[167] K. von Haeften, T. Laarmann, H. Wabnitz, T. Möller, Bubble formation and decay in ${ }^{3}$ He and ${ }^{4}$ He clusters, Phys. Rev. Lett. 88 (23) (2002) 233401.

[168] J. W. Keto, M. Stockton, W. A. Fitzsimmons, Dynamics of atomic and molecular states produced in electron-bombarded superfluid helium, Phys. Rev. Lett. 28 (13) (1972) 792-795.

[169] V. B. Eltsov, A. Y. Parshin, I. A. Todoshchenko, Optical spectra of ${ }^{4} \mathrm{He}_{2}^{*}$ triplet molecules in superfluid helium in magnetic fields, J. Exp. Theor. Phys. 81 (5) (1995) 909, (Zh. Eksp. Teor. Fiz. 108 (5) (1995) 1657-1667).

[170] V. B. Eltsov, S. N. Dzhosyuk, A. Y. Parshin, I. A. Todoshchenko, Spectroscopic studies of the triplet-state ${ }^{4} h e_{2}^{*}$ molecules in superfluid helium up to the solidification pressure, J. Low Temp. Phys. 110 (1-2) (1998) 219-224.

[171] S. G. Kafanov, A. Y. Parshin, I. A. Todoshchenko, Structure and dynamics of the $\operatorname{He}_{2}^{*}\left(a^{3} \sigma_{u}\right)$ molecular complex in condensed phases of helium, J. Exp. Theor. Phys. 91 (5) (2000) 991-999, (Zh. Eksp. Teor. Fiz. 118 (5) (2000) 1143-1152).

[172] A. V. Benderskii, J. Eloranta, R. Zadoyan, V. A. Apkarian, A direct interrogation of superfluidity on molecular scales, J. Chem. Phys. 117 (10) (2002) 1201-1213.

[173] D. B. Kopeliovich, A. Y. Parshin, S. V. Pereverzev, Spectral properties and dynamics of $\mathrm{He}_{2}^{*}$ excimer molecules in ${ }^{4} \mathrm{He}$ crystals, J. Exp. Theor. Phys. 69 (3) (1989) 638, (Zh. Eksp. Teor. Fiz. 96 (3) (1989) 1122-1136).

[174] F. Stienkemeier, J. Higgins, W. E. Ernst, G. Scoles, Laser spectroscopy of alkali-doped helium clusters, Phys. Rev. Lett. 74 (1995) 3592-3595.

[175] J. Higgins, C. Callegari, J. Reho, F. Stienkemeier, W. E. Ernst, M. Gutowski, G. Scoles, Helium cluster isolation spectroscopy of alkali dimers in the triplet manifold, J. Phys. Chem. A 102 (1998) 4952-4965.

[176] J. H. Reho, J. P. Higgins, K. K. Lehmann, Dynamics of the $1^{3} \Pi_{g}$ state of $K_{2}$ on helium nanodroplets, Faraday Discuss. 118 (2001) 33-42.

[177] F. R. Brühl, R. A. Miron, W. E. Ernst, Triplet states of rubidium dimers on helium nanodroplets, J. Chem. Phys. 115 (2001) 10275-10281.

[178] W. E. Ernst, R. Huber, S. Jiang, R. Beuc, M. Movre, G. Pichler, Cesium dimer spectroscopy on helium droplets, J. Chem. Phys. 124 (2006) 024313.

[179] M. Mudrich, O. Bünermann, F. Stienkemeier, O. Dulieu, M. Weidemüller, Formation of cold bialkali dimers on helium nanodroplets, Eur. Phys. J. D 31 (2004) 291-299.

[180] J. L. Persson, Q. Hui, M. Nakamura, M. Takami, Optical spectra of metal dimers and trimers in superfluid helium, Phys. Rev. A 52 (3) (1995) 2011-2015.

[181] V. V. Klimov, V. S. Letokhov, Enhancement and inhibition of spontaneous emission rates in nanobubbles, Chem. Phys. Lett. 301 (1999) 441.

[182] P. Moroshkin, A. Hofer, A. Weis, Lifetimes of Cs-He exciplexes in solid ${ }^{4} \mathrm{He}$, to be published.

[183] A. Weis, S. Kanorsky, S. Lang, T. W. Hänsch, Can paramagnetic atoms trapped in solid ${ }^{4}$ He be used to search for physics beyond the standard model?, Springer, 1997, Atomic Physics Methods in Modern Research, Lecture Notes in Physics, vol. 499, K. Jungmann, J. Kowalski, I. Reinhard, F. Träger.

[184] M. Arndt, S. I. Kanorsky, A. Weis, T. W. Hänsch, Long electronic spin relaxation times of Cs atoms in solid ${ }^{4} \mathrm{He}$, Phys. Rev. Lett 74 (8) (1995) 1359-1362.

[185] D. Budker, W. Gawlik, D. F. Kimball, S. M. Rochester, V. V. Yashchuk, A. Weis, Resonant nonlinear magneto-optical effects in atoms, Rev. Mod. Phys. 74 (2002) 1153-1201.

[186] A. Weis, G. Bison, A. S. Pazgalev, Theory of double resonance magnetometers based on atomic alignment, Phys. Rev. A 74 (3) (2006) 033401.

[187] S. Lang, S. I. Kanorsky, T. Eichler, R. Müller-Siebert, T. W. Hänsch, A. Weis, Optical pumping of Cs atoms in solid ${ }^{4}$ He, Phys. Rev. A 60 (5) (1999) 3867-3877.

[188] S. Lang, M. Arndt, T. W. Hänsch, S. I. Kanorsky, S. Lücke, S. B. Ross, A. Weis, Local field effects in the spectroscopy of Cs atoms trapped in solid ${ }^{4} \mathrm{He}$, Low Temp. Phys. 22 (2) (1996) 129-130, Fiz. Nizk. Temp., 22, 171-173 (1996).

[189] S. I. Kanorsky, S. Lang, S. Lücke, S. B. Ross, T. W. Hänsch, A. Weis, Millihertz magnetic resonance spectroscopy of Cs atoms in body-centered-cubic ${ }^{4} \mathrm{He}$, Phys. Rev. A 54 (2) (1996) R1010-R1013.

[190] S. Groeger, G. Bison, J. L. Schenker, R. Wynands, A. Weis, A high-sensitivity laser-pumped $\mathrm{M}_{x}$ magnetometer, Eur. Phys. J. D 38 (2006) 239-247. 
[191] T. Eichler, R. Müller-Siebert, D. Nettels, S. Kanorsky, A. Weis, Optical detection of nonradiating alkali atoms in solid helium, Phys. Rev. Lett. 88 (12) (2002) 123002.

[192] D. Nettels, R. Müller-Siebert, S. Ulzega, A. Weis, Multi-photon processes in the Zeeman structure of atomic Cs trapped in solid He, Appl. Phys. B 77 (2003) 563-570.

[193] D. Nettels, R. Müller-Siebert, A. Weis, Relaxation mechanism of muti-quantum coherences in the Zeeman structure of atomic Cs trapped in solid He, Appl. Phys. B 77 (2003) 753-764.

[194] T. Furukawa, Y. Matsuo, A. Hatekeyama, Y. Fukuyama, T. Kobayashi, H. Izumi, T. Shimoda, Measurement of a long electronic spin relaxation time of cesium atoms in superfluid helium, Phys. Rev. Lett. 96 (2) (2006) 095301.

[195] A. Hatakeyama, K. Oe, K. Ota, J. Arai, T. Yabuzaki, A. R. Young, Slow spin relaxation of Rb atoms confined in glass cells filled with dense ${ }^{4} \mathrm{He}$ gas at $1.85 \mathrm{~K}$, Phys. Rev. Lett. 84 (7) (2000) 1407-1410.

[196] J. P. Carrico, A. Adler, M. R. Baker, S. Legowski, E. Lipworth, P. G. H. Sandars, T. S. Stein, C. Weisskopf, Atomic beam resonance measurement of the differential polarizability between Zeeman substates in the ground state of the Cesium atom, Phys. Rev. 170 (1) (1968) 64.

[197] H. Gould, E. Lipworth, M. C. Weisskopf, Quadratic Stark shift between Zeeman substates in $\mathrm{Cs}^{133}, \mathrm{Rb}^{87}, \mathrm{Rb}^{85}, \mathrm{~K}^{39}$ and $\mathrm{Na}^{23}$, Phys. Rev. 188 (1) (1969) 24.

[198] C. Ospelkaus, U. Rasbach, A. Weis, Measurement of the forbidden tensor polarizability of Cs using an all-optical Ramsey resonance technique, Phys. Rev. A 67 (2003) 011402.

[199] S. Ulzega, A. Hofer, P. Moroshkin, R. Müller-Siebert, D. Nettels, A. Weis, Measurement of the forbidden electric tensor polarizability of Cs atoms trapped in solid ${ }^{4} \mathrm{He}$, Phys. Rev. A 75 (2007) 042505.

[200] S. Ulzega, Theoretical and experimental study of the Stark effect in the ground state of alkali atoms in He crystals, Ph.D. thesis, University of Fribourg, Switzerland, unpublished (2006).

[201] A. Hofer, P. Moroshkin, S. Ulzega, A. Weis, Calculation of the forbidden electric tensor polarizabilites of free Cs atoms and of Cs atoms trapped in a solid ${ }^{4}$ He matrix, Phys. Rev. A 77 (1) (2008) 012502.

[202] A. Abragam, Principles of nuclear magnetism, Oxford University Press, Oxford, 1961.

[203] D. Pines, C. P. Slichter, Relaxation times in magnetic resonance, Phys. Rev. 100 (4) (1955) 1014-1020.

[204] K. R. Atkins, Ions in liquid helium, Phys. Rev. 116 (6) (1959) 1339-1343.

[205] A. Phillips, P. V. E. McClintock, Field emission and field ionization in liquid ${ }^{4} \mathrm{He}$, Phil. Trans. Royal Soc. London A 278 (1281) (1975) 271-310.

[206] G. G. Ihas, T. M. Sanders, Injection and mobility of potassium ions in liquid helium, Phys. Lett. A 31 (9) (1970) $502-503$.

[207] W. W. Johnson, W. I. Glaberson, Positive impurity ions in He II, Phys. Rev. Lett. 29 (4) (1972) 214-217.

[208] H. Günther, M. Foerste, G. zu Putlitz, T. Schumacher, Ions in superfluid helium: Measurement of the roton limited mobility, Low Temp. Phys. 22 (2) (1996) 143-147, Fiz. Nizk. Temp., 22 (1996) 189-193.

[209] M. Foerste, H. Günther, O. Riediger, J. Wiebe, G. zu Putlitz, Ions and atoms in superfluid helium $\left({ }^{4}\right.$ He)IV. Temperature depenndence of the mobility of alkali earth ions, Z. Phys. B 104 (1997) 317.

[210] M. Foerste, H. Günther, O. Riediger, J. Wiebe, G. zu Putlitz, Temperature depenndent mobility measurements of alkaliearth ions in superfluid helium $\left({ }^{4} \mathrm{He}\right)$, J. Low Temp. Phys. 110 (1-2) (1998) 231-236.

[211] M. W. Cole, R. A. Bachman, Structure of positive impurity ions in liquid helium, Phys. Rev. B 15 (3) (1977) $1388-1394$.

[212] M. W. Cole, F. Toigo, Structural transition for positive impurity ions in fluids, Phys. Rev. B 17 (4) (1978) $2054-2056$.

[213] M. Himbert, A. Lezama, J. Dupont-Roc, Laser excitation of $\mathrm{Ba}^{+}$ions in liquid helium, J. Physique 46 (1985) $2009-2014$.

[214] A. Nakayama, K. Yamashita, Theoretical study on the structure of $\mathrm{Na}^{+}$-doped helium clusters: Path integral Monte Carlo calculations, J. Chem. Phys. 112 (24) (2000) 10966-10975.

[215] M. Buzzacchi, D. E. Galli, L. Reatto, Alkali ions in superfluid ${ }^{4} \mathrm{He}$ and structure of the snowball, Phys. Rev. B 64 (2001) 094512.

[216] D. E. Galli, M. Buzzacchi, L. Reatto, Pure and alkali-ion-doped droplets of ${ }^{4}$ He, J. Chem. Phys. 115 (22) (2001) 1023910247.

[217] M. Rossi, M. Verona, D. E. Galli, L. Reatto, Alkali and alkali-earth ions in ${ }^{4}$ He systems, Phys. Rev. B 69 (2004) 212510.

[218] F. Scaramuzzi, A. Savoia, D. L. Goodstein, M. W. Cole, Viscosity and positive-ion mobility near the melting transition in liquid ${ }^{4} \mathrm{He}$, Phys. Rev. B 16 (7) (1977) 3108-3121.

[219] M. W. Cole, T. J. Sluckin, Nucleation of freezing by charged particles. I. Thermodynamics, J. Chem. Phys. 67 (2) (1977) $746-750$.

[220] S. Balibar, H. Alles, A. Y. Parshin, The surface of helium crystals, Rev. Mod. Phys. 77 (2005) 317-370.

[221] K. O. Keshishev, Charge movement in crystalline helium, Zh. Eksp. Teor. Fiz. 72 (2) (1977) 521-544.

[222] K. O. Keshishev, A. E. Meierovich, Mobility of charges in crystalline helium in strong electric fields, Zh. Eksp. Teor. Fiz. 72 (5) (1977) 1953-1959.

[223] A. I. Golov, V. B. Yefimov, L. P. Mezhov-Deglin, Motion of injected charges in hexagonal closely packed ${ }^{4} \mathrm{He}$, Zh. Eksp. Teor. Fiz. 94 (2) (1988) 198-215.

[224] O. Andreeva, K. Keshishev, D. Kholin, P. Kossyrev, Charge transport in solid ${ }^{4}$ He, J. Low Temp. Phys. 110 (1-2) (1998) 89-96.

[225] S. C. Lau, A. J. Dahm, Nonlinear space-charge-limited ion currents in solid helium, J. Low Temp. Phys. 112 (1-2) (1998) $47-71$.

[226] E. B. Gordon, V. V. Khmelenko, A. A. Pelmenev, E. A. Popov, O. F. Pugachev, A. F. Shestakov, Metastable impurityhelium solid phase. Experimental and theoretical evidence, Chem. Phys. 170 (1993) 411-426. 
[227] R. E. Boltnev, G. Frossati, E. B. Gordon, I. N. Krushinskaya, E. A. Popov, A. Usenko, Embedding impurities into liquid helium, J. Low Temp. Phys. 127 (5-6) (2002) 245-258.

[228] E. B. Gordon, A. A. Pelmenev, E. A. Popov, O. F. Pugachev, V. V. Khmelenko, A. F. Shestakov, Cluster approximation analysis of impurity-helium phase formation and its stability, Fiz. Nizk. Temp. 18 (12) (1992) 1365-1374.

[229] E. A. Popov, J. Ahokas, J. Eloranta, H. Kunttu, On the formation mechanism of impurity-helium solids: evidence for extensive clustering, Low Temp. Phys. 29 (6) (2003) 510-514, (Fiz. Nizk. Temp. 29 (6) (2003) 684-689).

[230] E. B. Gordon, T. Kumada, M. Ishiguro, Y. Aratono, T. Momose, N. Nakashima, Doped helium crystals growth and study, J. Low Temp. Phys. 138 (3-4) (2005) 805-810.

[231] R. E. Boltnev, E. B. Gordon, V. V. Khmelenko, I. N. Krushinskaya, M. V. Martynenko, A. A. Pelmenev, E. A. Popov, A. F. Shestakov, Luminescence of nitrogen and neon atoms isolated in solid helium, Chem. Phys. 189 (1994) $367-382$.

[232] V. Kiryukhin, B. Keimer, R. E. B. V. V. Khmelenko, E. B. Gordon, Inert-gas solids with nanoscale porosity, Phys. Rev. Lett. 79 (9) (1997) 1774-1777.

[233] S. I. Kiselev, V. V. Khmelenko, D. M. Lee, V. Kiryukhin, R. E. Boltnev, E. B. Gordon, B. Keimer, Structural studies of impurity-helium solids, Phys. Rev. B 65 (2001) 024517.

[234] S. I. Kiselev, V. V. Khmelenko, D. M. Lee, V. Kiryukhin, R. E. Boltnev, E. B. Gordon, B. Keimer, X-ray studies of structural changes of impurity-helium solids, J. Low Temp. Phys. 126 (1-2) (2002) 235-240.

[235] S. I. Kiselev, V. V. Khmelenko, D. A. Geller, D. M. Lee, J. R. Beamish, Investigation of ultrasound propagation in porous impurity-helium solids, J. Low Temp. Phys. 119 (3-4) (2000) 357-366.

[236] S. I. Kiselev, V. V. Khmelenko, D. M. Lee, Investigation of ultrasound attenuation in impurity-helium solids containing liquid helium, J. Low Temp. Phys. 121 (5-6) (2000) 671-676.

[237] V. Kiryukhin, E. P. Bernard, V. V. Khmelenko, R. E. Boltnev, N. V. Krainyukova, D. M. Lee, Noble-gas nanoclusters with fivefold symmetry stabilized in superfluid helium, Phys. Rev. Lett. 98 (19) (2007) 195506.

[238] S. I. Kiselev, V. V. Khmelenko, D. M. Lee, Hydrogen atoms in impurity-helium solids, Phys. Rev. Lett. 89 (17) (2002) 175301.

[239] S. I. Kiselev, V. V. Khmelenko, D. M. Lee, C. Y. Lee, Magnetic resonance studies of impurity-helium solids containing hydrogen and deuterium impurities, J. Low Temp. Phys. 128 (1-2) (2002) 37-52.

[240] E. P. Bernard, R. E. Boltnev, V. V. Khmelenko, D. M. Lee, Long term stability of H atoms in HD-D 2 -He solids, J. Low Temp. Phys. 138 (3-4) (2005) 829-834.

[241] E. B. Gordon, A. A. Pelmenev, O. F. Pugachev, V. V. Khmelenko, Hydrogen and deuterium atoms stabilized by condensation of an atomic-beam in superfluid helium, J. Exp. Theor. Phys. Lett. 37 (5) (1983) 282-285, (Pis'ma Zh. Eksp. Teor. Fiz. 37 (5) (1983) 237-239).

[242] E. P. Bernard, R. E. Boltnev, V. V. Khmelenko, V. Kiryukhin, S. I. Kiselev, D. M. Lee, ESR and X-ray investigations of deuterium atoms and molecules in impurity-helium solids, J. Low Temp. Phys. 134 (1-2) (2004) 169-174.

[243] E. P. Bernard, R. E. Boltnev, V. V. Khmelenko, V. Kiryukhin, S. I. Kiselev, D. M. Lee, Deuterium atoms and molecules in nanoclusters of molecular deuterium, Phys. Rev. B 69 (10) (2004) 104201.

[244] E. A. Popov, J. Ahokas, J. Eloranta, H. Kunttu, Thermal decomposition mechanism of $\mathrm{N}_{2}$-impurity helium solids, J. Low Temp. Phys. 139 (5-6) (2005) 557-562.

[245] R. E. Boltnev, I. N. Krushinskaya, A. A. Pelmenev, D. Y. Stolyarov, V. V. Khmelenko, The thermoluminescence spectra obtained on the destruction of impurity-helium solid phase samples, Chem. Phys. Lett. 305 (1999) $217-224$.

[246] E. B. Gordon, A. A. Pelmenev, O. F. Pugachev, V. V. Khmelenko, The electron-spin-resonance studies of atoms trapped in liquid helium. I. Methods - the electron-spin-resonance spectra of nitrogen atoms, Fiz. Nizk. Temp. 8 (6) (1982) 601-607.

[247] E. P. Bernard, R. E. Boltnev, V. V. Khmelenko, D. M. Lee, Stabilization of high concentrations of nitrogen atoms in impurity-helium solids, J. Low Temp. Phys. 134 (1-2) (2004) 199-204.

[248] R. E. Boltnev, I. N. Krushinskaya, A. A. Pelmenev, E. A. Popov, D. Y. Stolyarov, V. V. Khmelenko, Study of the stabilization and recombination of nitrogen atoms in impurity-helium condensates, Low Temp. Phys. 31 (7) (2005) 547555, (Fiz. Nizk. Temp., 31 (7) (2005) 723-734).

[249] R. A. Aziz, S. S. Cohen, H. Dubost, M. L. Klein, Inert gases, Springer-Verlag, 1984.

[250] M. H. Edwards, Refractive index of ${ }^{4}$ He: Saturated vapor, Phys. Rev. 108 (1957) 1243-1245.

[251] P. Dietrich, N. Schwentner, Empirical Jahn-Teller potential surfaces for silver doped xenon matrices, J. Chem. Phys. 111 (5) (1999) 2133-2142.

[252] J. F. Dawson, L. C. Balling, Calculations of atomic level structure of alkali atoms in rare-gas solids, J. Chem. Phys. 71 (2) (1979) 836-842.

[253] L. C. Balling, J. J. Wright, Use of dimer potentials to calculate the energy levels of alkali atoms in rare-gas matrices, J. Chem. Phys. 79 (6) (1983) 2941-2944.

[254] J. A. Boatz, M. E. Fajardo, Monte Carlo simulations of the structures and optical absorption spectra of Na atoms in Ar clusters, surfaces, and solids, J. Chem. Phys. 101 (5) (1994) 3472-3487.

[255] M. E. Fajardo, Classical Monte Carlo simulations of relaxed trapping site structures in Li atom doped solid Ne, J. Chem. Phys. 98 (1) (1993) 119-125.

[256] J. Ahokas, T. Kiljunen, J. Eloranta, H. Kunttu, Theoretical analysis of alkali metal trapping sites in rare gas matrices, J. Chem. Phys. 112 (5) (2000) 2420-2426.

[257] W. Weyhmann, F. M. Pipkin, Optical absorption spectra of alkali atoms in rare-gas matrices, Phys. Rev. 137 (2) (1965) 490-496. 
[258] B. Meyer, Absorption spectrum of Na and K in rare-gas matrices, J. Chem. Phys. 43 (9) (1965) $2986-2992$.

[259] L. Andrews, G. C. Pimentel, Visible spectra of lithium in inert-gas matrices, J. Chem. Phys. 47 (8) (1967) $2905-2910$.

[260] H. Coufal, U. Nagel, M. Burger, E. Lüscher, Optical absorption spectra of $\mathrm{K}^{41}$-atoms isolated in argon matrices, Z. Phys. B 25 (1976) 227-235.

[261] L. C. Balling, M. D. Havey, J. F. Dawson, Absorption and emission spectra of Na atoms trapped in rare-gas matrices, J. Chem. Phys. 69 (4) (1978) 1670-1675.

[262] L. C. Balling, M. D. Havey, J. J. Wright, Absorption and emission spectra of K atoms trapped in rare-gas matrices, J. Chem. Phys. 70 (5) (1979) 2404-2408.

[263] J. J. Wright, L. C. Balling, Absorption and emission spectra of Li atoms trapped in rare-gas matrices, J. Chem. Phys. 73 (7) (1980) 3103-3106.

[264] L. C. Balling, J. J. Wright, Laser excitation of excited states of Rb and Cs atoms in an Ar matrix, J. Chem. Phys. 78 (1) (1983) 592-593.

[265] H. Kuppelmaier, H. J. Stöckmann, A. Sreinmetz, E. Görlach, H. Ackermann, Jahn-Teller effect of K atoms isolated in Ar matrices, Phys. Lett. A 98 (4) (1983) 187-191.

[266] P. A. Lund, D. Smith, S. M. Jacobs, P. N. Schatz, Magnetic circular dichroism study of the ${ }^{2} s \rightarrow^{2} p$ transition of lithium atoms in noble gas matrices, J. Phys. Chem. 88 (1984) 31-42.

[267] J. Rose, D. Smith, B. E. Williamson, P. N. Schatz, M. C. M. O'Brien, Magnetic circular dichroism and the Jahn-Teller effect in the ${ }^{2} s \rightarrow^{2} p$ transition of sodium and lithium atoms isolated in xenon matrices, J. Phys. Chem. 90 (1986) $2608-2615$.

[268] A. Schrimpf, G. Sulzer, H. J. Stöckmann, H. Ackermann, Correlation of electron spin resonance and optical absorption of $\mathrm{K}$ atoms in solid Ar, Z. Phys. B 67 (1987) 531-539.

[269] M. E. Fajardo, P. G. Carrick, J. W. Kenney, Matrix isolation spectroscopy of metal atoms generated by laser ablation. I The Li/Ar, Li/Kr, and Li/Xe systems, J. Chem. Phys. 94 (9) (1991) 5812-5825.

[270] M. E. Fajardo, Matrix isolation spectroscopy of metal atoms generated by laser ablation. II The Li/Ne, Li/D 2 , and Li/H 2 systems, J. Chem. Phys. 98 (1) (1993) 110-118.

[271] S. Tam, M. E. Fajardo, Matrix isolation spectroscopy of metal atoms generated by laser ablation. III The Na/Ar, Na/Kr, and Na/Xe systems, J. Chem. Phys. 99 (2) (1993) 854-860.

[272] D. C. Silverman, M. E. Fajardo, Matrix isolation spectroscopy of Na atoms deposited as Na ${ }^{+}$ions, J. Chem. Phys. 106 (22) (1997) 8964-8966.

[273] S. A. Mitchell, J. Farrell, G. A. K. Wallace G. A. Ozin, Optical emission and absorption studies of silver atoms in rare gas matrices at $12 \mathrm{~K}$; silver atom cryophotoaggregation, J. Am. Chem. Soc. 102 (1980) 7702-7709.

[274] W. Schulze, D. M. Kolb, H. Gerischer, Optical absorption spectra of matrix isolated silver atoms and their dependence on matrix properties, J. Chem. Soc. Faraday Trans. 71 (1975) 1763-1771.

[275] R. Kometer, N. Schwentner, Light-induced migration of Ag in Xe films and generation of sites, J. Chem. Phys. 104 (18) (1996) 6967-6975.

[276] M. Vala, K. Zeringue, J. ShakhsEmampour, J. C. Rivoal, R. Pyzalski, Magnetic circular dichroism studies of matrixisolated atoms: Excited state spin-orbit coupling constant reduction of copper in the noble gases, J. Chem. Phys. 80 (6) (1984) 2401-2406.

[277] D. Roser, R. Pellow, M. Eyring, M. Vala, J. Lignieres, J. C. Rivoal, Spin-orbit splitting and Jahn-Teller effect in rare gas matrix-isolated Ag and Au atoms: a magnetic circular dichroism study, Chem. Phys. 166 (1992) 393-409.

[278] J. T. Zoueu, M. Vala, J. C. Rivoal, Magneto-optical studies of silver atoms in neon matrices, Chem. Phys. 312 (2005) 89-96.

[279] K. Zeringue, J. ShakhsEmampour, J. C. Rivoal, M. Vala, Atom-matrix interaction: An MCD study of copper atoms in argon, J. Chem. Phys. 78 (5) (1983) 2231-2239.

[280] H. A. T. Vosbein, J. A. Boatz, J. W. Kenney, Moment analysis method as applied to the ${ }^{2} s \rightarrow^{2} p$ transition in cryogenic alkali metal/rare gas matrices, J. Phys. Chem. A 109 (2005) 11453-11461.

[281] J. W. Kenney, J. A. Boatz, H. A. T. Vosbein, Theory of Monte Carlo simulations of the magnetic circular dichroism spectra of alkali metal/rare gas systems, Int. J. Quant. Chem. 103 (2005) 854-865.

[282] J. H. Ammeter, D. C. Schlosnagle, Electronic quenching of Al and Ga atoms isolated in rare gas matrices, J. Chem. Phys. 59 (9) (1973) 4784-4820.

[283] R. Pellow, M. Vala, The external heavy atom effect: Theory of spin-orbit coupling of alkali and noble metals in rare gas matrices, J. Chem. Phys. 90 (10) (1989) 5612-5621.

[284] K. Vaskonen, J. Eloranta, H. Kunttu, Trapping of laser-vaporized alkali metal atoms in rare-gas matrices, Chem. Phys. Lett. 310 (1999) 245-251.

[285] M. C. M. O'Brien, Band shapes in Li/Xe: A very strongly coupled Jahn-Teller system, J. Chem. Phys. 82 (8) (1985) 3870-3871.

[286] D. M. Kolb, D. Leutloff, Fluorescence spectra of matrix isolated silver atoms, Chem. Phys. Lett. 55 (2) (1978) $264-266$.

[287] H. Wiggenhauser, W. Schroeder, D. M. Kolb, Energy dissipation in matrix-isolated silver atoms:A time-resolved fluorescence study, J. Chem. Phys. 88 (6) (1988) 3434-3440.

[288] R. Kometer, N. Schwentner, Dynamics of dissipation processes in the Ag:Xe complex, J. Chem. Phys. 106 (1) (1997) $51-59$.

[289] K. Bammel, P. Dietrich, N. Schwentner, Electron-lattice dynamics on Jahn-Teller surfaces in Ag:Xe crystals, J. Chem. Phys. 111 (5) (1999) 2123-2132. 
[290] C. Jeannin, M. T. Portella-Oberli, S. Jimenez, F. Vigliotti, B. Lang, M. Chergui, Femtosecond dynamics of electronic "bubbles" in solid argon: viewing the inertial response and the bath coherences, Chem. Phys. Lett. 316 (2000) 51-59.

[291] F. Vigliotti, L. Bonacina, M. Chergui, G. Rojas-Lorenzo, J. Rubayo-Soneira, Ultrafast expansion and vibrational coherences of electronic "bubbles" in solid neon, Chem. Phys. Lett. 362 (2002) 31-38.

[292] F. Vigliotti, L. Bonacina, M. Chergui, Ultrafast structural dynamics in electronically excited solid neon. I. Real-time probing of the electronic bubble formation, Phys. Rev. B 67 (11) (2003) 115118.

[293] S. Jimenez, M. Chergui, G. Rojas-Lorenzo, J. Rubayo-Soneira, The medium response to an impulsive redistribution of charge in solid argon: Molecular dynamics simulations and normal mode analysis, J. Chem. Phys. 114 (12) (2001) $5264-5272$.

[294] G. Rojas-Lorenzo, J. Rubayo-Soneira, F. Vigliotti, M. Chergui, Ultrafast structural dynamics in electronically excited solid neon. II. Molecular-dynamics simulations of the electronic bubble formation, Phys. Rev. B 67 (11) (2003) 115119.

[295] F. Vigliotti, M. Chergui, Rydberg states in the condensed phase studied by fluorescence depletion spectroscopy, Eur. Phys. J. D 10 (2000) 379-390.

[296] J. Eloranta, V. A. Apkarian, The triplet $\mathrm{He}_{2}^{*}$ Rydberg states and their interaction potentials with ground state He atoms, J. Chem. Phys. 115 (2) (2001) 752-760.

[297] J. Eloranta, Dynamics of He* triplet state excimer bubles in superfluid ${ }^{4} \mathrm{He}$, Chem. Phys. 332 (2007) $304-312$.

[298] C. K. Jen, V. A. Bowers, E. L. Cochran, S. N. Foner, Electron spin resonance of alkali atoms in inert-gas matrices, Phys. Rev. 126 (5) (1962) 1749-1757.

[299] J. P. Goldsborough, T. R. Koehler, Electron spin resonance studies of alkali metal trapping sites in solid rare gases, Phys. Rev. 133 (1A) (1964) 135-140.

[300] S. L. Kupferman, F. M. Pipkin, Properties of rubidium atoms trapped in a solid argon matrix, Phys. Rev. 166 (2) (1968) $207-218$.

[301] A. Schrimpf, R. Rosendahl, T. Bornemann, H. J. Stöckmann, F. Faller, L. Manceron, Thermally activated processes in Li doped Ar matrices studied by electronic spin-lattice relaxation, J. Chem. Phys. 96 (11) (1992) 7992-7999.

[302] F. J. Adrian, Matrix effects on the electron spin resonance spectra of trapped hydrogen atoms, J. Chem. Phys. 32 (4) (1960) 972-981.

[303] D. Y. Smith, Variational theory of paramagnetic impurities in van der Waals crystals, Phys. Rev. 131 (5) (1963) $2056-2069$.

[304] D. Y. Smith, Calculation of the g factor of hydrogen and the alkali atoms trapped in rare-gas solids, Phys. Rev. 133 (4A) (1964) 1087-1097.

[305] P. H. Kasai, D. McLeod, ESR studies of Cu, Ag, and Au atoms isolated in rare-gas matrices, J. Chem. Phys. 55 (4) (1971) $1566-1575$.

[306] M. Arndt, Optische Spektroskopie und Magnetresonanz an Metallatomen in flüssigem und festen (4)Helium, Ph.D. thesis, Ludwig Maximilians Universität München, Max-Planck-Institut für Quantenoptik, Garching, MPQ-Report 197 (1995).

[307] S. Lang, Optisch detektierte magnetresonanz an Cäsium atomen in festem ${ }^{4} \mathrm{He}$, Ph.D. thesis, Ludwig Maximilians Universität München, unpublished (1997).

[308] T. Eichler, Magneto-optical spectroscopy of alkali atoms in helium crystals, Ph.D. thesis, University of Bonn, Germany, unpublished (2000).

[309] D. Nettels, Optical magnetic resonance tomography and laser spectroscopy of cesium atoms trapped in solid helium, Ph.D. thesis, University of Fribourg, Switzerland, unpublished (2003).

[310] A. Hofer, Spectroscopic, time-resolved, and Stark effect studies of alkali atoms and exciplexes in solid ${ }^{4} \mathrm{He}, \mathrm{Ph} . \mathrm{D}$. thesis, University of Fribourg, Switzerland, unpublished (2007). 